\title{
D-optimal designs for prospective cohort studies
}

Citation for published version (APA):

Tekle, F. B. (2008). D-optimal designs for prospective cohort studies. [Doctoral Thesis, Maastricht University]. Datawyse / Universitaire Pers Maastricht. https://doi.org/10.26481/dis.20081008ft

Document status and date:

Published: 01/01/2008

DOI:

10.26481/dis.20081008ft

Document Version:

Publisher's PDF, also known as Version of record

\section{Please check the document version of this publication:}

- A submitted manuscript is the version of the article upon submission and before peer-review. There can be important differences between the submitted version and the official published version of record.

People interested in the research are advised to contact the author for the final version of the publication, or visit the DOI to the publisher's website.

- The final author version and the galley proof are versions of the publication after peer review.

- The final published version features the final layout of the paper including the volume, issue and page numbers.

Link to publication

\footnotetext{
General rights rights.

- You may freely distribute the URL identifying the publication in the public portal. please follow below link for the End User Agreement:

www.umlib.nl/taverne-license

Take down policy

If you believe that this document breaches copyright please contact us at:

repository@maastrichtuniversity.nl

providing details and we will investigate your claim.
}

Copyright and moral rights for the publications made accessible in the public portal are retained by the authors and/or other copyright owners and it is a condition of accessing publications that users recognise and abide by the legal requirements associated with these

- Users may download and print one copy of any publication from the public portal for the purpose of private study or research.

- You may not further distribute the material or use it for any profit-making activity or commercial gain

If the publication is distributed under the terms of Article $25 \mathrm{fa}$ of the Dutch Copyright Act, indicated by the "Taverne" license above, 


\section{D-optimal designs for prospective cohort studies}

By

Fetene Bekele Tekle 



\section{D-optimal designs for prospective cohort studies PROEFSCHRIFT}

Ter verkrijging van de graad van doctor aan de Universiteit Maastricht, op gezag van de Rector Magnificus, Prof. mr.G.P.M.F.Mols, volgens het besluit van het College van Decanen, in het openbaar te verdedigen op woensdag 8 oktober 2008 om 16:00 uur

door

Fetene Bekele Tekle 


\section{Promotor:}

Prof. dr. M.P.F. Berger

\section{Co-Promotor:}

Dr. E.S. Tan

\section{Beoordelingscommissie/Evaluation committee:}

Prof. dr. ir. P.A. van den Brandt (voorzitter)

Dr. G. van Breukelen

Prof. dr. P. Goos (Universiteit Antwerpen)

Prof. dr. G. Verbeke (Katholieke Universiteit Leuven)

Prof. dr. H. de Vries

(C) Fetene Bekele Tekle, Maastricht 2008

Universitaire Pers Maastricht

ISBN 978-90-5278-744-2

The studies presented in this thesis were supported by the Care and Public Health Research Institute (Caphri), Maastricht University. 
In memory of Ato Beyene Desta (Abaaba), Mimi and to W/o Fantaye W/yohannes (Emama) 



\section{Contents}

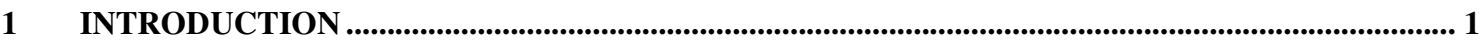

1.2 Generalized Linear Mixed Models (GLMM) …..............................................

1.2.1 The Linear Mixed Model ............................................................................................5

1.2.2 The Logistic Mixed model .......................................................................

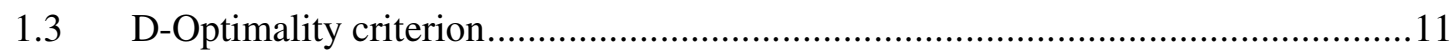

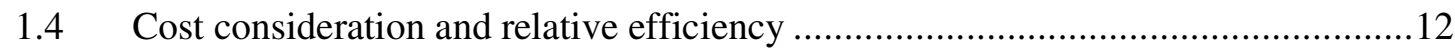

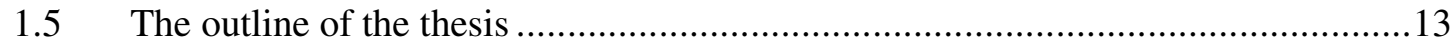

2 TOO MANY REPEATED MEASUREMENTS OVER TIME IS A WASTE OF RESOURCES ......15

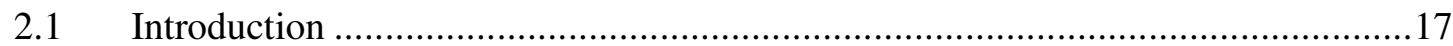

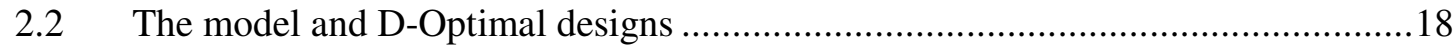

2.3 Cost consideration and relative efficiency ...................................................... 19

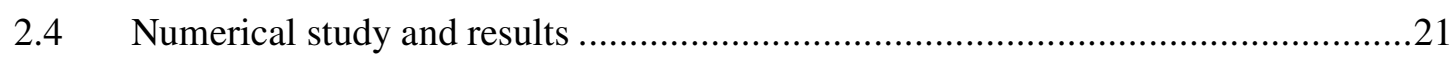

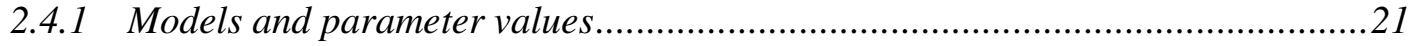

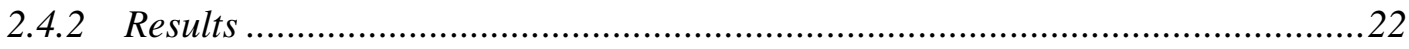

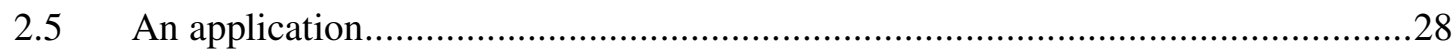

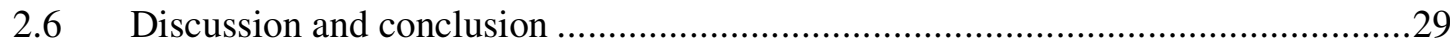

3 D-OPTIMAL COHORT DESIGNS FOR LINEAR MIXED EFFECTS MODELS ..............................33

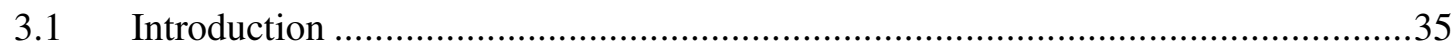

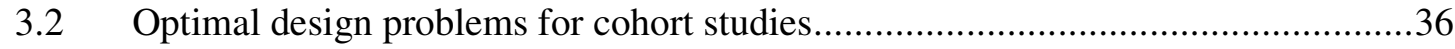

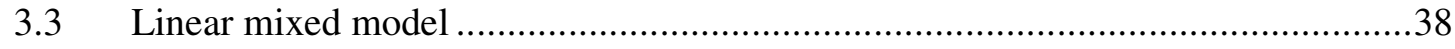

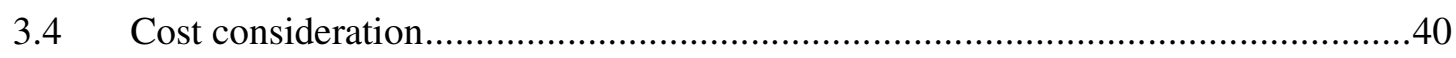

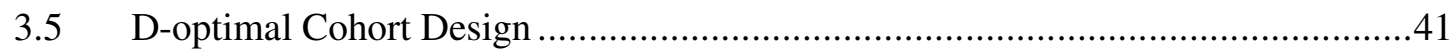

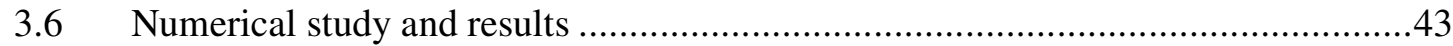

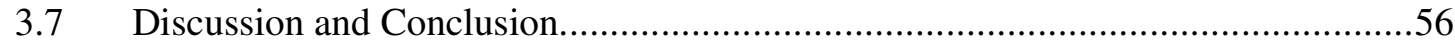

4 INTERACTIVE COMPUTER PROGRAM FOR OPTIMAL DESIGNS OF LONGITUDINAL

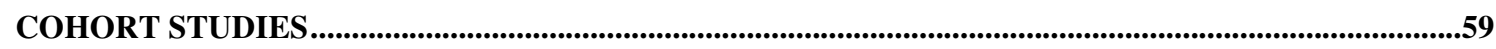

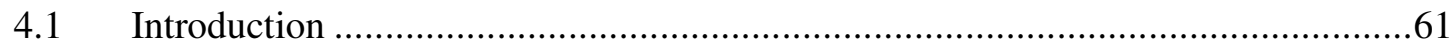

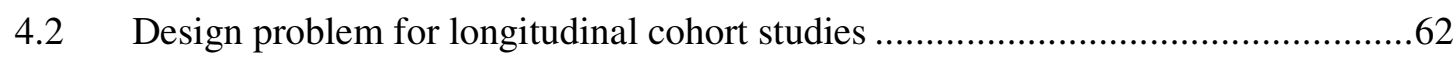

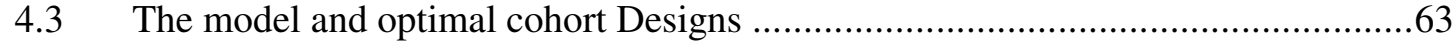

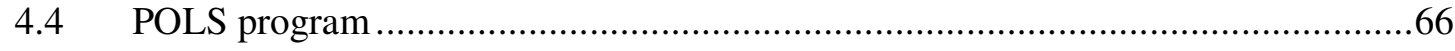

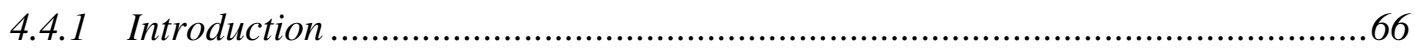

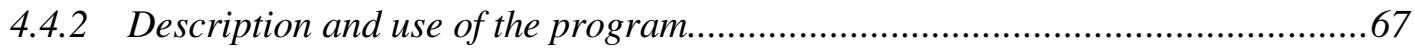

4.5 Illustration: Design on height of schoolgirls ...................................................... 70

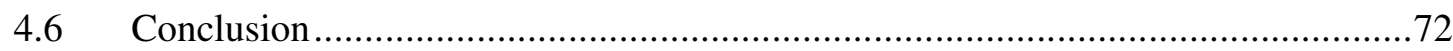




\section{HIGHLY EFFICIENT DESIGNS FOR LOGISTIC MODELS WITH CATEGORICAL}

VARIABLES .............................................................................................................................................................73

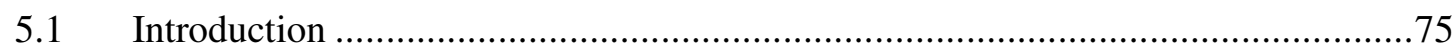

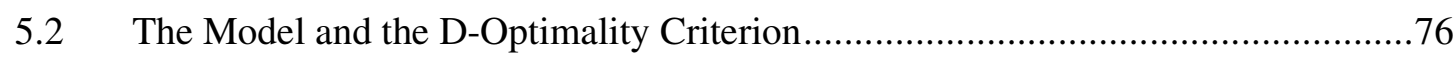

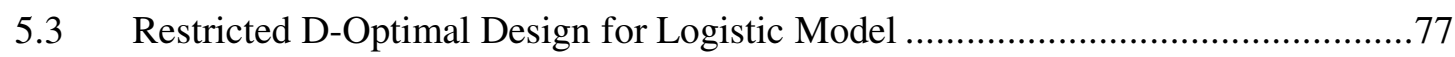

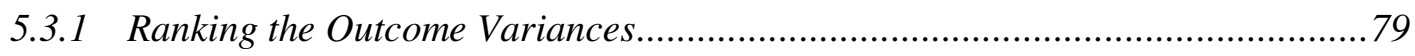

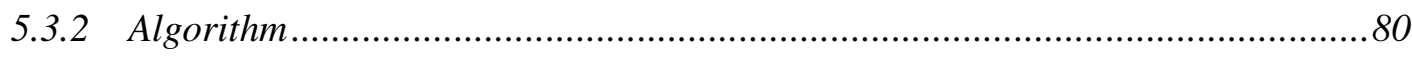

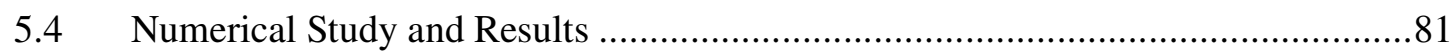

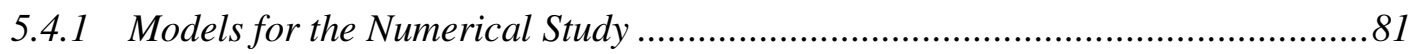

5.4.2 Model Parameters for the Numerical Study ............................................... 82

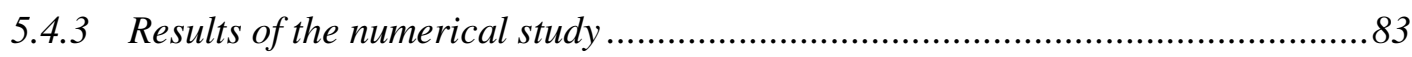

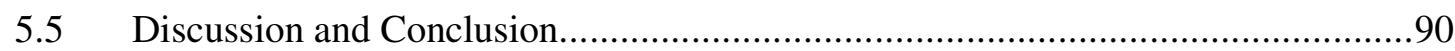

6 MAXIMIN D-OPTIMAL DESIGNS FOR BINARY LONGITUDINAL RESPONSES ......................93

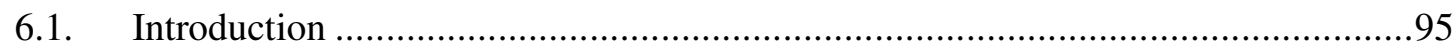

6.2. Logistic mixed-effects models and optimal designs ...........................................97

6.2.1. General logistic mixed-effects models........................................................97

6.2.2. variance-covariance matrix of the parameter estimators ..................................98

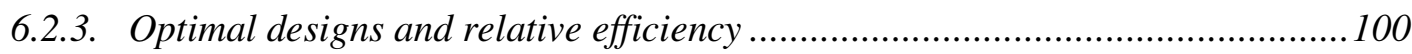

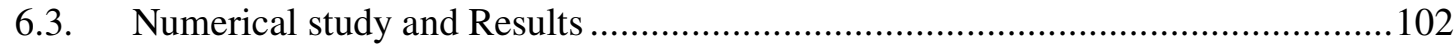

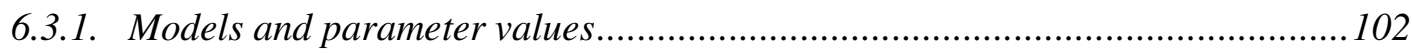

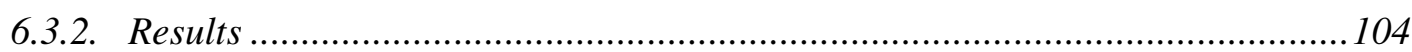

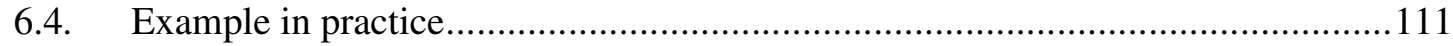

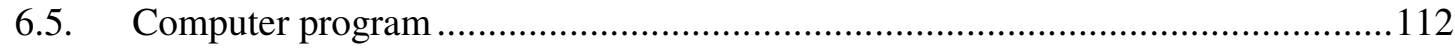

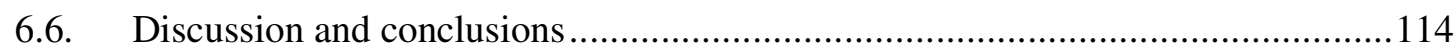

REFERENCES

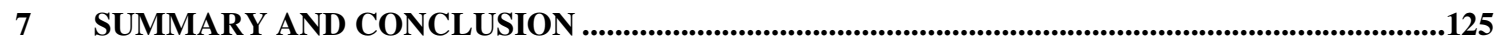

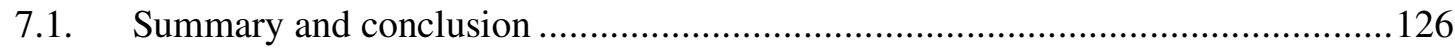

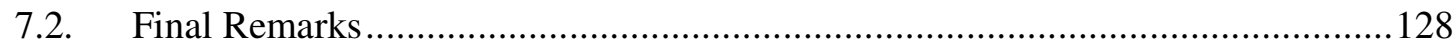

DUTCH SUMMARY (SAMENVATTING) ............................................................................................................130

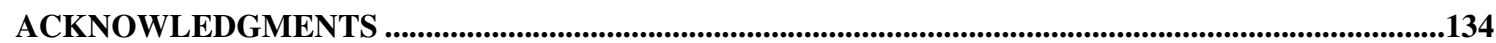

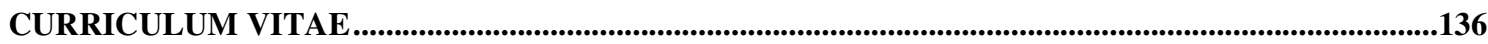


1 Introduction 


\section{Introduction}

Prospective cohort studies provide information about the same group of subjects at different points in time allowing the researcher to track change over time. In contrast to a single snapshot they are analogous to a photograph album, showing how individuals or groups have changed over time. For example, Potthoff and Roy (1964) studied the change over time in the distance from the center of the pituitary to the maxillary fissure of 27 children, 16 boys and 11 girls. On each child, the distance (mm) from the center of the pituitary to the maxillary fissure was made at ages $8,10,12$, and 14 years of age. Figure 1.1 shows the trend of 2 randomly selected boys. The complete data is reprinted by Verbeke and Molenberghs (2000). The expected trend is shown by the bold line in Figure 1.1, while the trends of each boy is shown by thin lines and the dots and stars show the observed data points for each child.

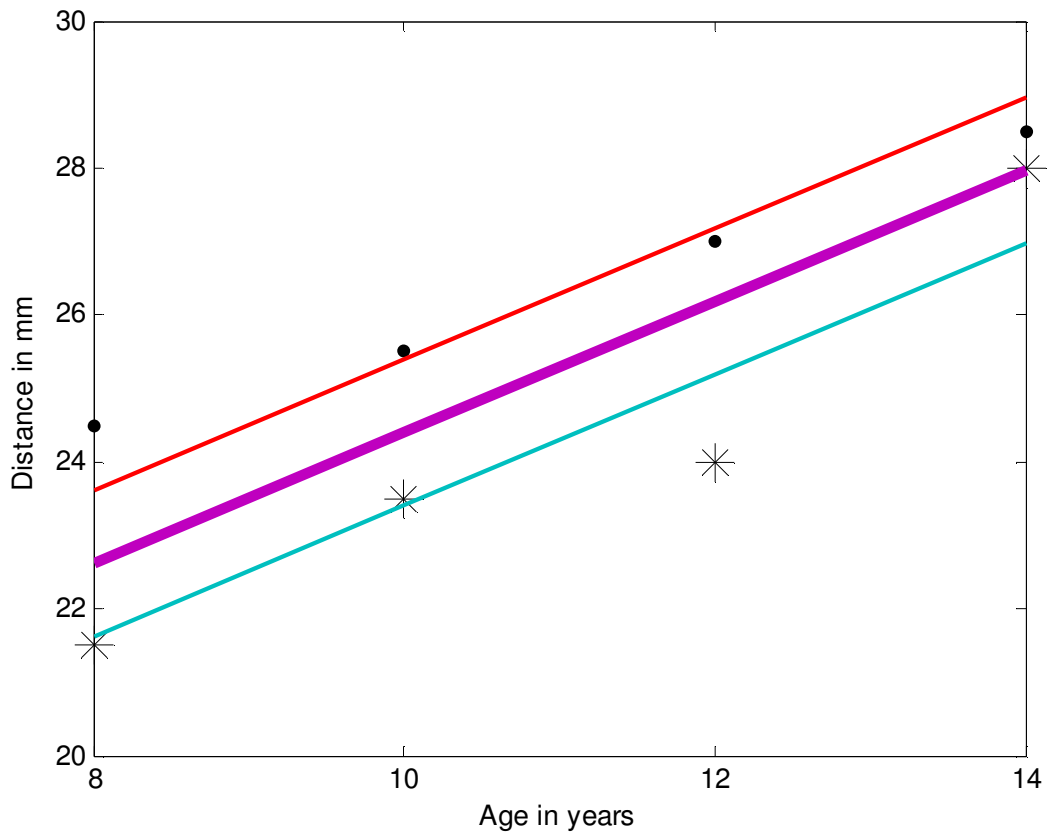

Figure 1.1. Growth data for 2 randomly selected boys.

Prospective cohort studies and longitudinal studies are synonymously used in this thesis. The major characteristic of longitudinal studies is that subjects are also measured on a certain specified outcome variable repeatedly over time. The outcome variable can be continuous or discrete and the statistical model for the analysis of data from longitudinal study designs is mostly chosen on the basis of the type of the outcome variable. Continuous and binary outcome variables are considered in this thesis. Hence, the linear 
mixed effects and logistic mixed effects models are assumed for the analysis of the data. These models are encompassed within the framework of the generalized linear mixed models (GLMM).

This thesis mainly focuses on optimal designs for longitudinal studies. Almost all optimality criteria in optimal design theory optimize a certain function of the variancecovariance matrix of the parameter estimators in the model. Hence, for the purpose of describing the variance-covariance matrix of the parameter estimators, the generalized linear mixed models are reviewed in section 1.1.

Many large-scale longitudinal studies have been set up in the past to identify associated risk factors on outcome measures over time. Some examples of such studies are the Framingham heart study (Dawber, 1980), the national cancer prevention study (Calle et al., 2002), the nurses' health study (Colditz, 1994; Camargo and Willett, 1999), the New York state cohort study (Bandera et al., 1997), the longitudinal aging study of Amsterdam (Dik et al., 2000), and the Survey of Medical Information and Life style study in Eindhoven (SMILE) (Bruijn et al., 2005; Van den Akker et al., 2008). These studies encounter irregular drop-out and are very expensive. The costs of these large scale studies may be reduced by choosing the design more carefully and by selecting samples more efficiently. Moreover, the number of repeated measures may also be selected more carefully to optimize the information. It seems therefore worthwhile to apply optimal design procedures to design these large scale longitudinal studies.

The purpose of this thesis is to identify and propose an optimal design in terms of number and allocations of time points for longitudinal studies with the constraint of study cost (budget). Applying optimal design theory in practice requires a criterion for comparing designs and an algorithm for optimizing the criterion over the set of possible designs. The commonly used optimality criteria are the E-, the A- and the D-optimality criterion and details of these criteria are described by Fedorov (1972), Silvey (1980) and Atkinson and Donev (1996). The basic idea underlying design optimality theory is that statistical inference about parameters of interest can be improved by optimally selecting levels of the control variables like time points of the measurement. A design which is optimal with respect to a criterion like D-optimality is the one that maximizes parameter information by minimizing variability of the parameter estimates. In this thesis, we will restrict ourselves to the most commonly used D-optimality criterion, so D-optimality will be described in section 1.2.

Researchers usually have a limited budget for a study and the number of repeated 
measurements per subject usually needs to be restricted. Therefore, it is reasonable to take the cost of measuring into account when a design is optimized and find the optimal number of measurements and the allocations of these measurements over time for the available fixed budget. Optimal designs with cost constraint are suggested in literature in the context of pharmacokinetics/Pharmacodynamics studies (Fedorov et al., 2002; Gagnon and Leonov, 2005). The current thesis will address the problem of optimal designs for longitudinal studies with cost consideration. This topic has not yet been fully considered before. Section 1.3 describes the possible costs that are encountered in longitudinal studies and relative efficiency that helps to compare different designs under a fixed total budget of a study.

\subsection{Generalized Linear Mixed Models (GLMM)}

One of the approaches to the analysis of longitudinal data is modeling the heterogeneity among subjects by using subject-specific (or random) effects. This approach considers mixed models where the random effects follow a certain assumed parametric distribution. Mixed linear models for continuous longitudinal data with Gaussian distribution of the outcome variable are popular and have been studied by Laird and Ware (1982), Diggle et al. (1994) and Verbeke and Molenberghs (2000) among others. Generalized Linear Mixed Models (GLMM) are also formulated as an extension of the Generalized Linear Model (GLM; McCullagh and Nelder, 1983) for longitudinal outcomes by Stiratelli et al. (1984), Liang and Zeger (1986), Lindsey (1993), Hand and Crowder (1996), McCulloch and Searle (2001) and Molenberghs and Verbeke (2005) among others. The formulation of the GLMM is briefly described below.

Let $y_{i j}$ be an outcome random variable and $\mathbf{x}_{i j}$ is a $p \times 1$ vector of fixed covariates at the $j^{\text {th }}$ time point for subject $i$, where $j=1, \cdots, q$ and $i=1, \cdots, N$. The number of time points $q$ can vary from subjects to subject; however, equal number of time points per subject (balanced design) is considered for simplicity. Let $\mathbf{z}_{i j}$ be an $r \times 1$ vector of covariates (typically a subset of $\mathbf{x}_{i j}$ ) associated with an $r \times 1$ random effect $\mathbf{b}_{i}$. Let the conditional mean be $E\left(y_{i j} \mid \mathbf{b}_{i}\right)=\mu_{i j}$ and the variance be $\operatorname{var}\left(y_{i j} \mid \mathbf{b}_{i}\right)=\phi v\left(\mu_{i j}\right)$, where $v(\cdot)$ is a specified variance function and $\phi$ is possibly a scale parameter which can be fixed at unity. The conditional mean is related to the linear predictor

$$
\eta_{i j}=\mathbf{x}_{i j}^{\prime} \boldsymbol{\beta}+\mathbf{z}_{i j}^{\prime} \mathbf{b}_{i}
$$


by the link function $g\left(\mu_{i j}\right)$ such that the conditional mean satisfies:

$$
\mu_{i j}=h\left(\mathbf{x}_{i j}^{\prime} \boldsymbol{\beta}+\mathbf{z}_{i j}^{\prime} \mathbf{b}_{i}\right),
$$

where $\boldsymbol{\beta}$ is a $p \times 1$ vector of fixed effects and $h(\cdot)$ is the inverse link function, i.e. $h=g^{-1}$. The model specification for the conditional mean is complete by assuming that the vector of the random effects, $\mathbf{b}_{i}$, has a multivariate normal distribution with mean $\mathbf{0}$ and covariance matrix $\mathbf{G}$ with size $r \times r$.

The type of the link function $g(\cdot)$ that can be used depends on the type of the outcome variable and the link function in turn determines the type of the specific model. Table 1.1 summarizes the corresponding link functions and related features for continuous and dichotomous (binary) outcome variables considered in this thesis. In Table $1.1, \pi_{i j}$ is the conditional probability of the dichotomous response variable $y_{i j}$.

Table 1.1. Two types of outcome variables and their corresponding model futures.

\begin{tabular}{cccccc}
\hline Model & $\begin{array}{c}\text { Type of outcome } \\
\text { variable }\end{array}$ & $\begin{array}{c}\text { Link function, } \\
g\left(\mu_{i j}\right)\end{array}$ & $\begin{array}{c}\text { Conditional } \\
\text { variance }\end{array}$ & $\begin{array}{c}\text { Inverse link } \\
\text { function, } h(\cdot)\end{array}$ & $\begin{array}{c}\text { Estimation } \\
\text { method }\end{array}$ \\
\hline Linear Mixed & Continuous & $\mu_{i j}$ & $\sigma^{2}$ & $\mathbf{x}_{i j}^{\prime} \boldsymbol{\beta}+\mathbf{z}_{i j}^{\prime} \mathbf{b}_{i}$ & MLE \\
Logistic Mixed & Dichotomous & $\ln \left(\frac{\mu_{i j}}{1-\mu_{i j}}\right)$ & $\begin{array}{c}\mu_{i j}\left(1-\mu_{i j}\right) \\
\text { or }\end{array}$ & $\frac{\exp \left(\mathbf{x}_{i j}^{\prime} \boldsymbol{\beta}+\mathbf{z}_{i j}^{\prime} \mathbf{b}_{i}\right)}{1+\exp \left(\mathbf{x}_{i j}^{\prime} \boldsymbol{\beta}+\mathbf{z}_{i j}^{\prime} \mathbf{b}_{i}\right)} \begin{array}{l}\text { PQL } \\
\text { and } \\
\text { extended GEE }\end{array}$ \\
& $\ln \left(\frac{\pi_{i j}}{1-\pi_{i j}}\right)$ & $\pi_{i j}\left(1-\pi_{i j}\right)$ & & \\
& & & & \\
\hline
\end{tabular}

As shown in Table 1.1, linear and logistic mixed models with the corresponding identity and logit links are the specific generalized linear mixed models for continuous and dichotomous outcome variables, respectively. The linear and logistic mixed models are described separately as follow.

\subsubsection{The Linear Mixed Model}

Linear mixed models may be expressed in different but equivalent forms. Denoting the measurement/observation vector by $\mathbf{y}_{i}=\left(y_{i 1}, \cdots, y_{i q}\right)^{\prime}$, the mean vector by $\boldsymbol{\mu}_{i}=\left(\mu_{i 1}, \cdots, \mu_{i q}\right)^{\prime}$ and the design matrices with rows $\mathbf{x}_{i j}^{\prime}$ and $\mathbf{z}_{i j}^{\prime}$ by $\mathbf{X}_{i}$ and $\mathbf{Z}_{i}$, respectively, the conditional mean in Equation (1.2) can be given by

$$
E\left(\mathbf{y}_{i} \mid \mathbf{b}_{i}\right)=\boldsymbol{\mu}_{i}=h\left(\mathbf{X}_{i} \boldsymbol{\beta}+\mathbf{Z}_{i} \mathbf{b}_{i}\right) \text {. }
$$

The vector of the outcome $\mathbf{y}_{i}$ can be decomposed as a linear function of the vectors of the 
conditional mean $\boldsymbol{\mu}_{i}$ and error terms. Using Equation (1.3) and the identity link shown in Table 1.1, the linear mixed effects model for each subject can be written as:

$$
\mathbf{y}_{i}=\boldsymbol{\mu}_{i}+\mathbf{e}_{i}=\mathbf{X}_{i} \boldsymbol{\beta}+\mathbf{Z}_{i} \mathbf{b}_{i}+\mathbf{e}_{i},
$$

where $\mathbf{X}_{i}$ is the $q \times p$ design matrix of fixed effects for subject $i, i=1, \cdots, N, \mathbf{Z}_{i}$ is the $q \times r$ design matrix of random effects for measurements of $i^{\text {th }}$ subject, and $\mathbf{e}_{i}$ is the $q \times 1$ vector of errors that follow a multivariate normal distribution with mean $\mathbf{0}$ and covariance matrix $\sigma^{2} \mathbf{R}$. The $q \times q$ matrix $\mathbf{R}$ is the correlation matrix of the error terms.

The product $\mathbf{X}_{i} \boldsymbol{\beta}$ in Equation (1.3) models the fixed part of $\mathbf{y}_{i}=\left(y_{i 1}, \cdots, y_{i q}\right)^{\prime}$ and $\mathbf{Z}_{i} \mathbf{b}_{i}+\mathbf{e}_{i}$ represents the random part of $\mathbf{y}_{i}$. It is assumed that the $q \times 1$ vectors of responses $\mathbf{y}_{i}$ of subject $i, i=1, \cdots, N$, are attributed to the expected trend $\mathbf{X}_{i} \boldsymbol{\beta}$ over all subjects, to the subject-specific deviations $\mathbf{Z}_{i} \mathbf{b}_{i}$ from this expected trend $\mathbf{X}_{i} \boldsymbol{\beta}$ and to the deviations $\mathbf{e}_{i}$ of the observations from the subject-specific profile $\mathbf{X}_{i} \boldsymbol{\beta}+\mathbf{Z}_{i} \mathbf{b}_{i}$.

In growth data, for example, the expected growth trend over all children can be modelled by the fixed effects (bold line in Figure 1.1), while the deviations of the growth trends of the children from the expected growth trend are modelled using additional childspecific parameters (deviation of thin lines from bold line), which we call random effects. Finally, for each child, the deviations of the observations from the growth trend of the children are modelled by adding a measurement error term (deviations of dots and star points from corresponding thin lines).

The vectors of the random-effects $\mathbf{b}_{i}$ and the measurement errors $\mathbf{e}_{i}$ are assumed to be independently, normally distributed with mean vectors $\mathbf{0}$ and covariance matrices $\mathbf{G}$ and $\sigma^{2} \mathbf{R}$, respectively. The structure of $\mathbf{G}$ specifies the type of random effects included in the model. We will consider fixed effects, random intercept and random slope parameters in this thesis. The first order autoregressive $(\mathrm{AR}(1))$ correlation structure is assumed for the error terms, i.e. for the $q(q-1) / 2$ elements of $\mathbf{R}$, the $\left(j, j^{\prime}\right)$ element is equal to $\sigma^{2} \rho^{\left|t_{j}-t_{j}\right|}$ for $j \neq j^{\prime}$, where $\rho$ is the serial correlation coefficient and $t_{j}$ and $t_{j^{\prime}}$ are the time points at which the measurements $j$ and $j$ of the subject are taken. This implies that the correlation is a decreasing function of the time between two measurements.

If we consider the random part $\mathbf{Z}_{i} \mathbf{b}_{i}+\mathbf{e}_{i}$ of $\mathbf{y}_{i}$ as nuisance from the expected trend $\mathbf{X}_{i} \boldsymbol{\beta}$, then the variance-covariance matrix of the responses of subject $i$ is attributed to (Verbeke and Molenberghs, 2000) 


$$
\mathbf{V}_{i}=\operatorname{var}\left(\mathbf{y}_{i}\right)=\left\{\left[\mathbf{Z}_{i} \operatorname{var}\left(\mathbf{b}_{i} \mathbf{Z}_{i}^{\prime}\right]+\operatorname{var}\left(\boldsymbol{\varepsilon}_{i}\right)\right\}=\left[\left(\mathbf{Z}_{i} \mathbf{G} \mathbf{Z}_{i}^{\prime}\right)+\sigma^{2} \mathbf{R}\right] .\right.
$$

The classical approach to estimation and inference on the regression parameters and variance-covariance parameters is based on maximum likelihood (ML). Assuming that the data have a multivariate normal distribution and independence across subjects, the likelihood for linear mixed effects model has the form (Molenberghs and Verbeke, 2005, p. 39):

$$
\mathrm{L}\left(\boldsymbol{\beta}, \mathbf{V}_{i}\right)=\prod_{i=1}^{N}\left\{(2 \pi)^{-\frac{q}{2}} \operatorname{Det}\left(\mathbf{V}_{i}\right)^{-\frac{1}{2}} \times \exp \left[-\frac{1}{2}\left(\mathbf{y}_{i}-\mathbf{X}_{i} \boldsymbol{\beta}\right)^{\prime} \mathbf{V}_{i}^{-1}\left(\mathbf{y}_{i}-\mathbf{X}_{i} \boldsymbol{\beta}\right)\right]\right\},
$$

where $\operatorname{Det}(\cdot)$ is the determinant function of a matrix. Since estimation of the fixed effects parameters is usually of primary interest, we will focus on optimal designs for the estimation of the fixed effects parameters $\boldsymbol{\beta}$. The maximum likelihood estimator (MLE) of the fixed effects parameters $\boldsymbol{\beta}$ can be obtained by maximizing the likelihood in Equation (1.6) with respect to $\boldsymbol{\beta}$ and by solving the system of normal equations or score functions. Conditional on $\mathbf{V}_{i}$, the MLE of $\boldsymbol{\beta}$ and its variance-covariance are given by:

$$
\hat{\boldsymbol{\beta}}=\left(\sum_{i=1}^{N} \mathbf{X}_{i} \mathbf{V}_{i}^{-1} \mathbf{X}_{i}\right)^{-1} \sum_{i=1}^{N} \mathbf{X}_{i} \mathbf{V}_{i}^{-1} \mathbf{y}_{i}
$$

and

$$
\operatorname{var}(\hat{\boldsymbol{\beta}})=\left(\sum_{i=1}^{N} \mathbf{X}_{i} \mathbf{V}_{i}^{-1} \mathbf{X}_{i}\right)^{-1}
$$

It is assumed that the covariance parameters in $\mathbf{V}_{i}$ are known in Equations (1.7) and (1.8); in practice, however, the covariance parameters are unknown and the covariance matrix $\mathbf{V}_{i}$ itself has to be estimated first. We refer to Diggle et al. (1994) and Verbeke and Molenberghs (2000) for the two frequently used methods, maximum likelihood (ML) and restricted maximum likelihood (REML) for the estimation of $\mathbf{V}_{i}$.

\subsubsection{The Logistic Mixed model}

As described in Table 1.1, if the outcome variable $y_{i j}$ at $j^{\text {th }}$ measurement for subject $i$ is on a binary (dichotomous) scale, the logistic mixed model is appropriate instead of the linear mixed model described in the previous section. We will assume that, conditional on all fixed and random effects in the model, the responses $y_{i j}$ are independently and Bernouli distributed with conditional probabilities $\pi_{i j}$. These probabilities are related to the linear 
predictor via the logit link and the corresponding logistic mixed effects model is given by:

$$
\operatorname{logit}\left(\pi_{i j}\right)=\ln \left(\frac{\pi_{i j}}{1-\pi_{i j}}\right)=\mathbf{x}_{i j}^{\prime} \boldsymbol{\beta}+\mathbf{z}_{i j}^{\prime} \mathbf{b}_{i},
$$

Where the vectors $\mathbf{x}_{i j}, \boldsymbol{\beta}, \mathbf{z}_{i j}$ and $\mathbf{b}_{i}$ are as defined earlier. Hence, the conditional probability, $\pi_{i j}$, can be obtained using the inverse link function as:

$$
\pi_{i j}=\frac{\exp \left(\mathbf{x}_{i j}^{\prime} \boldsymbol{\beta}+\mathbf{z}_{i j}^{\prime} \mathbf{b}_{i}\right)}{1+\exp \left(\mathbf{x}_{i j}^{\prime} \boldsymbol{\beta}+\mathbf{z}_{i j}^{\prime} \mathbf{b}_{i}\right)} .
$$

The corresponding likelihood function for the logistic mixed effects model is given by (Molenberghs and Verbeke, 2005, p. 267):

$$
\mathrm{L}(\boldsymbol{\beta}, \mathbf{G})=\prod_{i=1}^{N} \int_{-\infty}^{\infty}\left(\prod_{j=1}^{q} f_{i j}\left(y_{i j} \mid \mathbf{b}_{i}, \boldsymbol{\beta}\right)\right) f\left(\mathbf{b}_{i} \mid \mathbf{G}\right) d \mathbf{b}_{i},
$$

where the density $f_{i j}$ is the conditional density of the response $y_{i j}$, which is Bernoulli density for binary responses, and the density $f$ is the density of the vector of random effects $\mathbf{b}_{i}$ which is multivariate normal distribution. Thus, the likelihood function is the marginal density of the outcome variable obtained from the joint density of the outcome variable and the random effects by integrating with respect to the random effects.

For binary responses, the likelihood function in Equation (1.11) is computed by integrating a product of Bernouli and normal densities. Analytic expression for Equation (1.11) is available for continuous responses with Gaussian density. In general, no analytic expressions are available and numerical approximations are required. There is a large statistical literature (Pinheiro and Bates, 2000; Skrondal and Rabe-Hesketh, 2004; Molenberghs and Verbeke, 2005 among others) on various methods to the numerical approximations of Equation (1.11). Here, we will restrict ourselves to the penalised quasilikelihood (PQL, Breslow and Clayton, 1993) method which is implemented in most statistical software packages.

\section{PQL method for logistic mixed effects model}

Breslow and Clayton (1993) used Laplace methods for the integral approximation of the log-likelihood function of the data and obtained PQL. They defined a working response vector $\mathbf{y}_{i}^{*}$ with elements $y_{i j}^{*}=\eta_{i j}+\left(y_{i j}-\mu_{i j}\right) g^{\prime}\left(\mu_{i j}\right)$, where $\eta_{i j}$ is the linear predictor given in (1.1) and $g^{\prime}$ is the derivative of the link function $g$; here $g^{\prime}\left(\mu_{i j}\right)=1 / \mu_{i j}\left(1-\mu_{i j}\right)$ for the logit link function. An iterative weighted least-squares algorithm can be applied to 
estimate the fixed effects and then the random-effects parameters based on linear model theory for the working response vector. Thus, $\mathrm{PQL}$ is an approximate method that involves repeated fitting of linear mixed models with working response variables and iterative weights that depend on parameter estimates from previous step of iteration. As mentioned earlier the variance-covariance matrix of the parameter estimator is important for optimal design theory. Here, we give the expression for the variance-covariance matrix of the parameter estimators of the vector of the fixed effects parameters and refer to Breslow and Clayton (1993) for details on the procedures of PQL. An approximate variance-covariance matrix for the estimator of $\boldsymbol{\beta}$ at the end of the iterative steps of the PQL method is given by:

$$
\operatorname{var}(\hat{\boldsymbol{\beta}}) \approx\left(\sum_{i=1}^{N} \mathbf{X}_{i} \mathbf{V}_{i}^{-1} \mathbf{X}_{i}\right)^{-1},
$$

where the $q \times q$ variance-covariance matrix of the responses, $\mathbf{V}_{i}$, is approximated by:

$$
\mathbf{V}_{i} \approx \mathbf{W}_{i}^{-1}+\mathbf{Z}_{i} \mathbf{G} \mathbf{Z}_{i}^{\prime}
$$

where $\mathbf{W}_{i}$ is a diagonal matrix of the conditional variances of the outcomes given the random-effects $\mathbf{b}_{i}$, i.e.

$$
\mathbf{W}_{i}=\operatorname{diag}\left(w_{i 1}^{\mathbf{b}_{i}}, \cdots, w_{i q}^{b_{i}}\right),
$$

where $w_{i j}^{\mathbf{b}_{i}}=v\left(\pi_{i j}\right)=\pi_{i j}\left(1-\pi_{i j}\right)$, for $i=1, \cdots, N, j=1, \cdots q, v(\cdot)$ is a variance function for binary responses (McCullagh and Nelder, 1989). Note that the approximation in Equation (1.13) is similar to the one given in Equation (1.8) for the continuous outcome variable with normal distributions. This similarity is not strange as the PQL method also uses normal theory on a linear model on the working response vector of the outcome variable.

\section{The logistic mixed model with serial correlation}

The formulation of the logistic mixed model in the previous section and its corresponding PQL method of estimation does not allow for the serial correlations between repeated measurements. Zeger et al. (1988) and Molenberghs and Verbeke (2005) have shown that the serial correlation can also be included to the mixed model with non-Gaussian responses. They have extended the generalized estimating equation (GEE, Zeger et al., 1986) approach for the estimation of the parameters in a mixed effects model with serial correlation. We will briefly discuss the model and the extended GEE approach below in the context of the variance-covariance matrix of the parameter estimators and refer to Zeger et al. (1988) and Molenberghs and Verbeke (2005) for more details. 
The model given in Equation (1.9) describes the relationship between the conditional mean (probability) and the linear predictor. A basic model similar to Equation (1.4) for the vector of binary outcomes as a decomposition of the mean and the error structures can be given by:

$$
\mathbf{y}_{i}=\boldsymbol{\pi}_{i}+\mathbf{e}_{i}
$$

where $\mathbf{y}_{i}$ is a $q \times 1$ vector with binary outcomes $y_{i j}, \boldsymbol{\pi}_{i}$ is a $q \times 1$ vector with elements $\pi_{i j}$ as specified in Equation (1.10), for $j=1, \cdots, q$ and $\mathbf{e}_{i}$ is a $q \times 1$ vector of the error terms. These error terms at subject level can be allowed to be correlated and the resulting model will be a logistic mixed model with serial correlation. Zeger et al. (1988) have shown that the marginal moments can be approximated from the conditional moments and the distribution of the random effects can be used in the generalized estimating equations (GEE) of Linag and Zeger (1986). Then, the parameters can be estimated by solving the GEE. Zeger et al. (1988) and Molenberghs and Verbeke (2005) gave an approximate equation for the variance-covariance matrix of the parameter estimator for the generalized linear mixed model with serial correlation. The approximate variance-covariance matrix of the parameter estimators for the logistic mixed model with serial correlation given in Equation (1.15) is:

$$
\operatorname{var}(\hat{\boldsymbol{\beta}}) \approx\left(\sum_{i=1}^{N} \mathbf{X}_{i}^{\prime} \mathbf{W}_{i} \mathbf{V}_{i}^{-1} \mathbf{W}_{i}^{\prime} \mathbf{X}_{i}\right)^{-1}
$$

where the variance-covariance of the responses $\mathbf{V}_{i}$ is given by:

$$
\mathbf{V}_{i} \approx \mathbf{W}_{i}^{1 / 2} \mathbf{R} \mathbf{W}_{i}^{1 / 2}+\mathbf{W}_{i} \mathbf{Z}_{i} \mathbf{G} \mathbf{Z}_{i}^{\prime} \mathbf{W}_{i}^{\prime}
$$

where $\mathbf{W}_{i}$ is the diagonal matrix of the conditional variances of the responses given the random effect $\mathbf{b}_{i}=0$, i. e.

$$
\mathbf{W}_{i}=\operatorname{diag}\left(w_{i 1}^{0}, \cdots, w_{i q}^{0}\right),
$$

where $w_{i j}^{0}==v\left(\pi_{i j_{\mathbf{b}_{i}=\mathbf{0}}}\right)=\pi_{i j_{\mathbf{b}_{i}=\mathbf{0}}}\left(1-\pi_{i j_{\mathbf{b}_{i}=\mathbf{0}}}\right)$, for $i=1, \cdots, N, j=1, \cdots q, v(\cdot)$ is a variance function for binary responses (McCullagh and Nelder, 1989). The $q \times q$ matrix $\mathbf{R}$ is the correlation matrix of the error terms (see Molenberghs and Verbeke (2005) chapters 22 and 24 for details). 


\subsection{D-Optimality criterion}

Even if different methods of estimation are used in the analysis of longitudinal data, the accuracy of the estimators depends upon the number and timing of samples, as reflected in the variance-covariance of the parameter estimators via the design matrix. The purpose of this thesis is to identify and propose optimal designs in terms of number and allocations of time points for longitudinal studies in the context of linear and logistic mixed models with constraints. We will focus on the problem of designing a longitudinal study to estimate the fixed effect parameters of the mixed model, rather than the random effect parameters. We will use a D-optimality criterion to approach the problem.

The D-optimality criterion is the most well known and widely used design criteria. If the model parameter vector is denoted by $\boldsymbol{\beta}, \mathrm{D}$-optimality suggests that the choice of design should maximize the information on $\boldsymbol{\beta}$ by minimizing the determinant of the variance-covariance matrix of the parameter estimators in $\hat{\boldsymbol{\beta}}$ where $\hat{\boldsymbol{\beta}}$ is the estimator of $\boldsymbol{\beta}$. The asymptotic variance- covariance matrix of $\hat{\boldsymbol{\beta}}$ forms the foundation for traditional design optimality criteria.

The D-optimality criterion, which minimizes the generalized variance of $\hat{\boldsymbol{\beta}}$, is defined as

$$
\min _{\xi \in \Xi} \operatorname{Det}\left\{\operatorname{var}\left(\hat{\boldsymbol{\beta}}_{\xi}\right)\right\},
$$

where $\hat{\boldsymbol{\beta}}_{\xi}$ is the estimator of $\boldsymbol{\beta}$ under design $\xi$ and $\Xi$ is the set of all possible designs. The D-optimal design minimizes the volume of the asymptotic confidence ellipsoid of the parameter estimators. The volume of the confidence ellipsoid reflects how well $\boldsymbol{\beta}$ is estimated. Obviously, a smaller volume implies better estimation. D-optimal designs also have a nice property that they do not depend on the chosen time interval $[a, b]$, where a and $\mathrm{b}$ are the two end points of the time interval, i.e., if a linear transformation is considered on the time interval, the D-optimal designs can be obtained for the new time interval by applying the same transformation (Ouwens et al., 2006).

The D-optimality criterion which is commonly used in linear models continues to be used in nonlinear models and generalized linear mixed effects model. However, unlike designs for linear models, those for the latter models depend on the unknown parameters of the fitted model. In this case, the construction of a design would unfortunately require some knowledge of the parameters. Common approaches to solving this design 
dependence problem are:

a) The specification of initial values of the unknown parameters and the subsequent determination of so-called locally optimal designs (Sitter and $\mathrm{Wu}, 1993$ and Mathew and Sinha, 2001).

b) The sequential approach whereby updated estimates of the parameters are obtained in successive stages of the design process (Wu, 1985; Sitter and Forbes, 1997; and Sitter and $\mathrm{Wu}, 1999)$.

c) The Bayesian approach where a prior distribution is assumed on the parameters, which is then incorporated into an appropriate design criterion by integrating it over the prior distribution (Zacks, 1977; Dette and Sperlich, 1994; Mukhopadhyay and Haines, 1995; Chaloner and Larntz, 1989; Chaloner and Verdinelli,1995; Dasgupta, 1996; Khuri et al., 2006).

d) Maximin/minimax approach which minimizes the worst possible case over a user selected region of parameter values (King and Wong, 2000; Ouwens et al., 2002, 2006; Braess and Dette, 2007). In addition to the locally D-optimal designs we will consider the maximin approach for designs of binary longitudinal responses in chapter 6 .

\subsection{Cost consideration and relative efficiency}

There are two main components of the costs of obtaining repeated measurements for subjects. These are the costs of recruiting a subject and the costs of the repeated measurements once a subject is recruited. Suppose that the costs of the initial setup of a longitudinal study is $c_{0}$. This quantity $c_{0}$ may also include the costs of maintaining the research staff during the study period. Let the costs of recruiting a new subject be $c_{1}$, and the costs of obtaining a repeated measurement per subject be $c_{2}$. Then the total costs (budget) of a study with $q$ repeated measurements for $N$ subjects may be represented by the cost function

$$
C=c_{0}+N\left[c_{1}+c_{2}(q-1)\right] .
$$

The total costs of a study depends on the number of repeated measurements, i.e., the costs 
of measuring a subject with many repeated measurements may be more than the costs of measuring a subject with relatively few repeated measurements. Thus, to fairly compare different designs we will consider designs with the same costs of measurement. This means that the number of subjects $N$ and the number of repeated measurements $q$ may vary in the comparison.

The relative efficiency (RE) can be used to compare two designs. The relative efficiency of design $\xi_{2}$ with $q_{2}$ repeated measurements compared to design $\xi_{1}$ with $q_{1}$ repeated measurements is given by:

$$
\begin{aligned}
\operatorname{RE}_{\xi_{2} \mid \xi_{1}} & =\frac{\left[c_{1}+c_{2}\left(q_{1}-1\right)\right]}{\left[c_{1}+c_{2}\left(q_{2}-1\right)\right]}\left[\frac{\operatorname{Det}\left\{\operatorname{var}\left(\hat{\boldsymbol{\beta}}_{\xi_{1}}\right)\right\}}{\operatorname{Det}\left\{\operatorname{var}\left(\hat{\boldsymbol{\beta}}_{\xi_{2}}\right)\right\}}\right]^{1 / p} \\
& =\frac{\left[k+\left(q_{1}-1\right)\right]}{\left[k+\left(q_{2}-1\right)\right]}\left[\frac{\operatorname{Det}\left\{\operatorname{var}\left(\hat{\boldsymbol{\beta}}_{\xi_{1}}\right)\right\}}{\operatorname{Det}\left\{\operatorname{var}\left(\hat{\boldsymbol{\beta}}_{\xi_{2}}\right)\right\}}\right]^{1 / p},
\end{aligned}
$$

where $k=c_{1} / c_{2}$ is the cost ratio which plays a role in comparison of two designs for a fixed total budget. Note that when $k=1$, the costs of recruiting a new subject is equal to the costs of an additional measurement and Equation (1.21) reduces to the expression given for RE by Tan and Berger (1999) and Moerbeek (2005) where the RE of different designs is computed for fixed number of total measurements. The inverse RE represents the number of times design $\xi_{2}$ needs to be replicated to have the same value of the determinant of the covariance matrix as design $\xi_{1}$ (Atkinson and Donev, 1996).

\subsection{The outline of the thesis}

Each of the next chapters in this thesis may be read as a self-contained article and some texts in one chapter may be repeated in other chapters. The notations and the formulae may slightly deviate in one chapter to another to facilitate explanation of a specific problem and to increase readability. A brief introduction to the chapters is given as follows.

Chapter 2. In this chapter we proceed the work of Tan and Berger (1999). They have computed D-optimal designs for linear, quadratic, and cubic random intercept models as a function of the autocorrelation coefficient and compared the optimal designs with designs having equally spaced time points. Since nowadays mixed effects models are frequently used to analyse fixed and random effects in cohort studies, we extend the results for general linear mixed models with cost considerations. 
Chapter 3. This chapter is an extension of chapter 2 and the work of Berger (1986). Unlike chapter 2, the number of cohorts is not limited to one. Berger (1986) compared purely longitudinal, mixed longitudinal and cross-sectional cohort designs based on balanced designs. We extended the comparison of these cohort designs based on the Doptimality criterion. The other extension is consideration of a cost function in the comparison of these designs. The objective is to compare D- optimal cohort designs under cost constraint, and give recommendations to planners of large scale cohort studies.

Chapter 4. A new user friendly computer program is introduced and described in this chapter. In the past few years, a number of papers have been published on optimal designs for longitudinal studies. However, there is no computer-program that helps longitudinal study planners to easily get the results they need based on optimal design theory. The objective is to introduce and describe our new user-friendly computer-program for the optimization of designs for longitudinal cohort studies based on the methods discussed in chapters 2 and 3 .

Chapter 5. As mentioned in section 1.2, optimal designs for logistic models generally require prior information about the values of the regression parameters. The objective of chapter 5 is to search for highly efficient designs that are not dependent on model parameters. We proposed a design for a fixed effect logistic model which is D-optimal on a restricted design region and that assigns an equal weight to design points which contain more information and ignores those design points which contain less information about the regression parameters. We have shown how to construct the design in practice.

Chapter 6. Optimal design problems for logistic mixed-effects models for binary longitudinal responses are considered in this chapter. Locally D-optimal designs for logistic mixed-effects models are investigated and the maximin D-optimal is used to overcome the problem of parameter dependency. A user friendly computer program to obtain locally D-optimal and maximin D-optimal designs for binary longitudinal responses is described.

The thesis ends with a short summary and conclusion in Chapter 7. Some remarks and suggested future works are also given in the last chapter. 
2 Too Many Repeated Measurements over Time is a Waste of Resources

Submitted for publication 


\begin{abstract}
:
Researchers in Health Science and Medicine often use cohort designs for the study of change of outcome over time. It is common that these large scale cohort studies encounter irregular drop-out, and that they are very expensive. The costs of these studies may be reduced by choosing the design including the number of time points more carefully and by selecting samples more efficiently. It is therefore worthwhile to apply optimal design procedures to design such large scale cohort studies. The D-optimality criterion is used to construct optimal designs for mixed effects models. A linear cost function for repeated measurements over time is proposed, and this cost function is applied in the optimization procedure. It is found that in most practical cases a reduced number of repeated measurements leads to a gain in efficiency for parameter estimators. Taking a reduced number of repeated measurements may result in loss of efficiency when the costs of adding a new subject is much higher than the costs of adding an additional measurement per subject and the measurements are not correlated. However, this efficiency loss is not much. The results are illustrated using a cohort study from the literature.
\end{abstract}

Key words: D-optimal designs, Cohort design, Relative efficiency, Longitudinal study, Repeated measurements, Costs, Mixed effects model 


\subsection{Introduction}

Researchers in Health Sciences and Medicine are often interested in estimating changes over time. For example, the change of health status over time can be estimated by a repeated measurement design with measurements of health status at different time points. In the past, many large-scale longitudinal (cohort) studies have been set up for this purpose. Examples of such studies are the Framingham Heart Study (Dawber, 1980), the National Cancer Prevention Study (Calle et al., 2002), the Nurses' Health Study (Colditz, 1994), the Longitudinal Aging Study of Amsterdam (Dik et al., 2000), a prospective cohort study on diet and cancer in the Netherlands (van den Brandt and Bausch-Goldbohm, 1993) and the Survey of Medical Information and Life Style Study in Eindhoven (SMILE) (Van den Akker et al., 2008). These large scale studies all have in common that different cohorts of people are measured several times over a longer period of time. The national cancer prevention study, for example, used 68000 volunteers and measured them over a time span of 12 years (Calle et al., 2002), and the SMILE project selects samples from about 35000 patients registered in general practitioners offices and plans to measure them yearly over 10 to 15 years. These studies encounter irregular drop-out and are very expensive. The costs of these large scale studies may be reduced by choosing the design more carefully and by selecting samples more efficiently. Moreover, the number of repeated measures may also be selected more carefully to optimize the information. It seems therefore worthwhile to apply optimal design procedures to design these large scale cohort studies.

A researcher designing a longitudinal study has to decide on the number and allocation of time points. A series of papers focused on the optimal number and allocation of time points for polynomial growth models. Abt et al. (1997) gave optimal designs for the slope parameter estimation and growth prediction for linear growth model with intraclass and autocorrelated error structures. Abt et al. (1998) also studied A-, D-, and E-optimal designs for the quadratic growth model. Chang and Lay (2002) also studied the optimal allocation of time points for a specific growth curve model. Moerbeek (2005) studied robustness properties of A-, D-, and E-optimal designs for growth curve models with autocorrelated errors. In all these papers, polynomial fixed effect growth curve models are assumed. Tan and Berger (1999) computed D-optimal designs for linear, quadratic, and cubic random intercept models as a function of autocorrelation coefficient and compared the optimal 
designs with designs having equally spaced time points. Winkens et al. $(2005,2006,2007)$ have studied optimal designs for randomized clinical trials with linear and quadratic divergent treatment effects.

Since nowadays mixed effects models are frequently used to analyse fixed and random effects in cohort studies, we will extend the results for the literature and study optimal designs for cohort studies based on linear mixed models. In optimizing the designs, we will also consider a linear cost function. We will demonstrate how the application of optimal design techniques can lead to a substantial reduction in the number of measurements per subject, without loss of efficiency, and with substantial costs savings. In section 2.2, the linear mixed effects model and optimal designs will be described. A cost function is proposed and discussed in section 2.3. Relative efficiency is defined as a measure of comparison of two designs in the same section. In section 2.4, a numerical study is given to find optimal designs at specific cost parameters for different polynomial mixed effects models. Finally, discussion and conclusion are given in section 2.5.

\subsection{The model and D-Optimal designs}

Let $q$ be the number of time points per subject and let each subject be measured once at each time point. A polynomial mixed effects regression model for subject $i$ can be expressed as (Cnaan et al., 1997; Verbeke and Molenberghs, 2000):

$$
\mathbf{y}_{i}=\mathbf{X}_{i} \boldsymbol{\beta}+\mathbf{Z}_{i} \mathbf{b}_{i}+\mathbf{e}_{i}
$$

where $\mathbf{y}_{i}$ is the $q \times 1$ vector of quantitative responses or outcome for subject $i$, $i=1, \cdots, N, \quad \mathbf{X}_{i}$ is the $q \times p$ design matrix of fixed effects whose $j^{\text {th }}$ row is $\mathbf{x}\left(t_{i j}\right)=\left(1, t_{i j}, \cdots, t_{i j}^{(p-1)}\right)^{\prime}$ with polynomial terms of time $t_{i j}$ which is the time point for repeated measurement $j, j=1, \cdots, q, \boldsymbol{\beta}=\left(\beta_{0}, \beta_{1}, \cdots, \beta_{p-1}\right)^{\prime}$ is the $p \times 1$ vector of fixed effects regression coefficients, $\mathbf{Z}_{i}$ is the $q \times r$ design matrix of the random effects whose $j^{\text {th }}$ row $\mathbf{z}\left(t_{i j}\right)=\left(1, t_{i j}, \cdots, t_{i j}^{r-1}\right)^{\prime}$ is a sub vector of $\mathbf{x}\left(t_{i j}\right), \mathbf{b}_{i}=\left(b_{0 i}, b_{1 i}, \cdots, b_{(r-1) i}\right)^{\prime}$ is the $r \times 1$ vector of subject specific or random effects regression coefficients with zero mean and $r \times r$ covariance matrix $\mathbf{G}$, the vector $\mathbf{e}_{i}$ of random errors has zero mean and $q \times q$ covariance matrix $\sigma^{2} \mathbf{R}$.

Since fixed effects estimation is usually of primary interest, we will focus on the optimal designs for the estimation of the fixed effects. Given the parameter values in 
covariance matrices $\mathbf{G}$ and $\mathbf{R}$, the maximum likelihood estimator of the vector of fixed effects regression coefficients is:

$$
\hat{\boldsymbol{\beta}}=\left\{\sum_{i} \mathbf{X}_{i}^{\prime}\left(\mathbf{Z}_{i} \mathbf{G} \mathbf{Z}_{i}^{\prime}+\mathbf{R}_{i}\right)^{-1} \mathbf{X}_{i}\right\} \sum_{i} \mathbf{X}_{i}^{\prime}\left(\mathbf{Z}_{i} \mathbf{G} \mathbf{Z}_{i}^{\prime}+\sigma^{2} \mathbf{R}\right)^{-1} \mathbf{y}_{i},
$$

with asymptotic covariance matrix

$$
\operatorname{var}(\hat{\boldsymbol{\beta}})=\left\{\sum_{i} \mathbf{X}_{i}^{\prime}\left(\mathbf{Z}_{i} \mathbf{G} \mathbf{Z}_{i}^{\prime}+\sigma^{2} \mathbf{R}\right)^{-1} \mathbf{X}_{i}\right\}^{-1} .
$$

A design $\xi_{q}$ is an element of the design space $\Xi$ with all possible designs having $q$ time points. The amount of information in a design is measured by the Fisher information matrix, which is equal to the inverse of the asymptotic covariance matrix of the parameter estimators. The optimal design $\xi_{q}^{*}$ is the design among all possible designs $\xi_{q}$ with $q$ time points for which a function of the covariance matrix is minimized. The most commonly used function of the covariance matrix is the determinant function and the corresponding optimality criterion is known as the D-optimality criterion. We will denote a D-optimality criterion for the $\xi_{q}$ designs by $\mathrm{D}_{q}$. The $\mathrm{D}_{q}$-optimal design minimizes the determinant of the covariance matrix, $\operatorname{det}\left\{\operatorname{var}\left(\hat{\boldsymbol{\beta}}_{\xi_{q}}\right)\right\}$, where $\operatorname{var}\left(\hat{\boldsymbol{\beta}}_{\xi_{q}}\right)$ is the asymptotic covariance of the parameter estimators in equation (2.3) under the design $\xi_{q}$. We will restrict ourselves to this criterion as it has some nice properties. It is equal to minimizing the volume of the confidence ellipsoid of the parameter estimators. $\mathrm{D}_{q}$-optimal designs also have a nice property that they do not depend on the chosen time interval [a, b], i.e., a linear transformation of the time interval leaves the D-optimal design unchanged.

\subsection{Cost consideration and relative efficiency}

Taking more measurements per subject obviously provides additional information and increases the precision of parameter estimators. Still, the number of repeated measurements that may be taken for each subject should be restricted in practice because of an increased probability of drop-out, and for logistical and ethical reasons. The inclusion of an additional observation increases the costs of the study. Therefore, it is reasonable to take the costs of measuring and analyses into account when the design is evaluated in terms of efficiency.

Suppose that the costs of the initial setup of a cohort study which also includes the 
costs of maintaining the research during the study period is $c_{0}$, that the costs of recruiting a new subject is $c_{1}$, and the costs of obtaining an additional measurement per subject is $c_{2}$. Then the total costs of the study with $q$ repeated measurements for $N$ subjects can be given by the linear cost function:

$$
C=c_{0}+N\left\{c_{1}+c_{2}(q-1)\right\} .
$$

Note that the total costs $\mathrm{C}$ will usually be large as compared to $c_{0}$, because the sample size $N$ is large. We will assume that recruiting a new subject costs the same or more than the costs of obtaining an additional measurement per subject, i.e. we will consider cases where the cost ratio is $k=c_{1} / c_{2} \geq 1$.

To fairly compare different designs we will consider designs with the same costs of measurement $C-c_{0}=N\left\{c_{1}+c_{2}(q-1)\right\}$. This means that the number of subjects $N$ and the number of repeated measurements $q$ may vary in the comparison. First, optimal designs for a given number of time points is identified and then the optimal designs for different number of time points and a total costs are compared. It is known that when $k=c_{1} / c_{2}=1$, an optimal design is a design with $q=p$ time points, and that this optimal design is more efficient than the $\mathrm{D}_{q}$-optimal design $\xi_{q}^{*}$ with $q>p$ time points (see Tan and Berger (1999) among others). In the present article, the more general cost ratio $k=c_{1} / c_{2} \geq 1$ will be considered in comparing an optimal design with $q>p$ time points and optimal design with $q=p$ time points. Let $\xi_{p}^{*}$ be the optimal design with $p=q$ time points, and $\xi_{q}^{*}$ be the optimal design with $q>p$ time points. Then relative efficiency (RE) of an optimal design $\xi_{q}^{*}$ compared to an optimal design $\xi_{p}^{*}$ is given by (Tan and Berger, 1999; Moerbeek, 2005):

$$
\mathrm{RE}_{\xi_{q}^{*} \xi_{p}^{*}}=\frac{\left\{c_{1}+c_{2}(p-1)\right\}}{\left\{c_{1}+c_{2}(q-1)\right\}}\left\{\frac{\operatorname{det}\left\{\operatorname{var}\left(\hat{\boldsymbol{\beta}}_{\xi_{p}^{*}}\right)\right\}}{\operatorname{det}\left\{\operatorname{var}\left(\hat{\boldsymbol{\beta}}_{\xi_{q}^{*}}\right)\right\}}\right\}^{1 / p} .
$$

Note that the inverse RE represents the number of times design $\xi_{q}^{*}$ needs to be replicated to have the same value of the determinant of the covariance matrix as design $\xi_{p}^{*}$ (Atkinson and Donev, 1996). $\mathrm{RE}_{\xi_{q}^{*} \xi_{p}^{*}}<1$ implies that the design $\xi_{p}^{*}$ is more efficient than design $\xi_{q}^{*}$. It is assumed in equation (2.5) that data will be available for all the subjects in the study period. 
From equation (2.5), the total costs of the study $C$, and the initial setting up costs $c_{0}$ will not play a role in the comparison of two designs. The RE in (2.5) depends on the values of the costs $c_{1}, c_{2}$, the numbers of time points $p$ and $q$, and the determinant of the covariance matrix of the regression parameters of the model. Note that the covariance matrix in (2.3) depends on the design matrix $\mathbf{X}$, the parameter values in the covariance matrices $\mathbf{G}$ and $\mathbf{R}$. In the next section a numerical study is described for different values of the covariance matrices $\mathbf{G}$ and $\mathbf{R}$.

\subsection{Numerical study and results}

\subsubsection{Models and parameter values}

A number of different models and parameter values were included in our numerical study. Mixed effects models with linear $(p=2)$, quadratic $(p=3)$, and cubic $(p=4)$ polynomials that are common in practice were considered. We have the following four classes of models:

- The model in equation (2.1) reduces to a fixed effect model with autocorrelated errors when the covariance matrix $\mathbf{G}_{1}=\mathbf{0}$.

- A random intercept model with the covariance matrix $\mathbf{G}_{2}=\left[\begin{array}{ll}1 & 0 \\ 0 & 0\end{array}\right]$. We have fixed the variance of the random intercept $g_{11}=1$ without loss of generality.

- A random intercept and random slope model with the covariance matrix $\mathbf{G}_{3}=\left[\begin{array}{cc}1 & 0 \\ 0 & 0.25\end{array}\right]$. The variances of random intercept $g_{11}=1$ and random slope $g_{22}=0.25$. The correlation between random intercept and random slope is assumed to be zero.

- A random intercept and random slope model with covariance matrix $\mathbf{G}_{4}=\left[\begin{array}{ll}1 & 0.5 \sqrt{0.25} \\ 0.5 \sqrt{0.25} & 0.25\end{array}\right]$, i.e. a random intercept and random slope model with correlation between the random intercept and random slope $r_{12}=0.5$. Note that the covariance between random intercept and random slope $g_{12}=r_{12} \sqrt{g_{11} g_{22}}$.

A literature scan of longitudinal studies showed that in many studies the variance of the 
random errors $\sigma^{2}$ is often less than the variance of the random intercept $g_{11}$ (see for example results of vorozole study, prostate data, rat data and height of school girls in Verbeke and Molenberghs (2000)). Therefore we have chosen $\sigma^{2}=0.25$ in this numerical study. A first order autoregressive (AR(1)) correlation structure is assumed for the error terms, i.e. the $\left(j, j^{\prime}\right)$ element of the covariance matrix $\mathbf{R}$ is equal to $\sigma^{2} \rho^{\left|t_{j}-t_{j^{\prime}}\right|}$, where $\rho$ is the autocorrelation coefficient that gives the correlation between two time points that are one unit of time apart. Since time structured data are likely to be positively correlated, values $\rho \in[0.00,0.95]$ are considered.

The cost ratio $k=c_{1} / c_{2}$ is the relative size of the costs of adding a new subject to the costs of an additional observation per subject. Values $k=1,2,5,10$ and 20 are considered. This means that, for instance when $k=10$, the costs of recruiting a new subject is ten times more expensive than the costs of an additional measurement per subject.

Since a linear transformation of the time interval leaves the D-optimal design unchanged, the time interval for the optimization procedure can be arbitrarily fixed to [-1, 1]. Many large scale longitudinal studies often measure the subjects over more than 5 to 10 time points. Therefore, we restricted the number of time points to $q \leq 15$. Since the patterns of the allocation of time points and RE are similar for different values of the number of time points $q$, we will only present the results for number of time points $q=p, 5,10$, and 15.

\subsubsection{Results}

\subsubsection{Optimal allocations of time points}

The optimal allocations of time points for models with the different covariance structures $\mathbf{G}$ of the random parameters are almost the same. Therefore, we have only presented the optimal allocations of $q$ time points for the random intercept and random slope model with covariance matrix $\mathbf{G}_{4}$ in Figure 2.1.

The plots in the columns in Figure 2.1 represent the D-optimal design points corresponding to polynomial models with $p=2$, 3, and 4. Each set of row plots corresponds to a different number of time points $q=p, q=5, q=10$, and $q=15$ respectively. In each plot, the horizontal axis represents the D-optimal design points and the vertical axis corresponds to the autocorrelation parameter $\rho$. Tan and Berger (1999) 
have reported similar plots for the optimal allocations of time points for the fixed effects model only. It can be seen from the plots that the optimal time points for linear and quadratic models are approximately equally spaced, provided that the serial correlation $\rho$ is not too small (i.e. $\rho>0.10$ ). Although the optimal time points for cubic models are not equally spaced in general, Figure 2.1 shows that, as the number of time points $q$ increases and the serial correlation is not too small $(\rho>0.10)$, they all become approximately equally spaced.

linear $(p=2)$
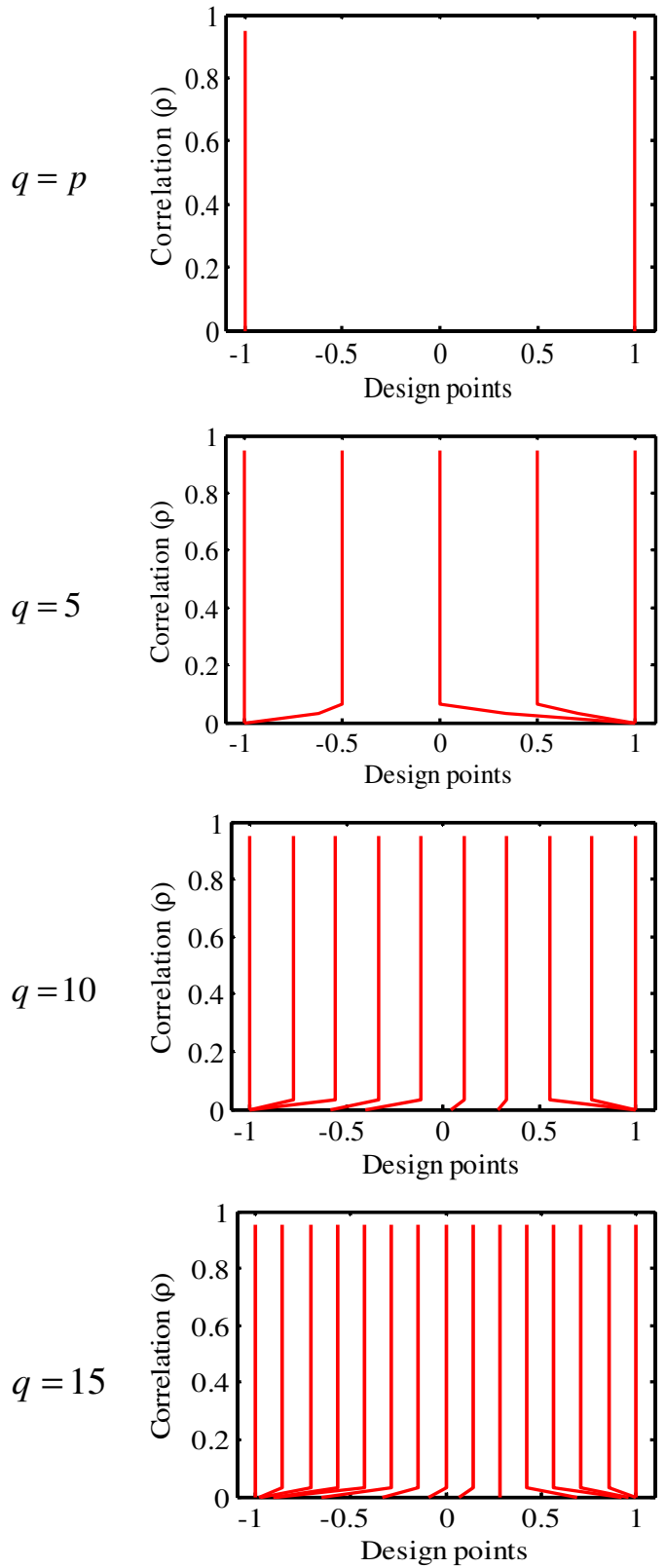

quadratic $(p=3)$
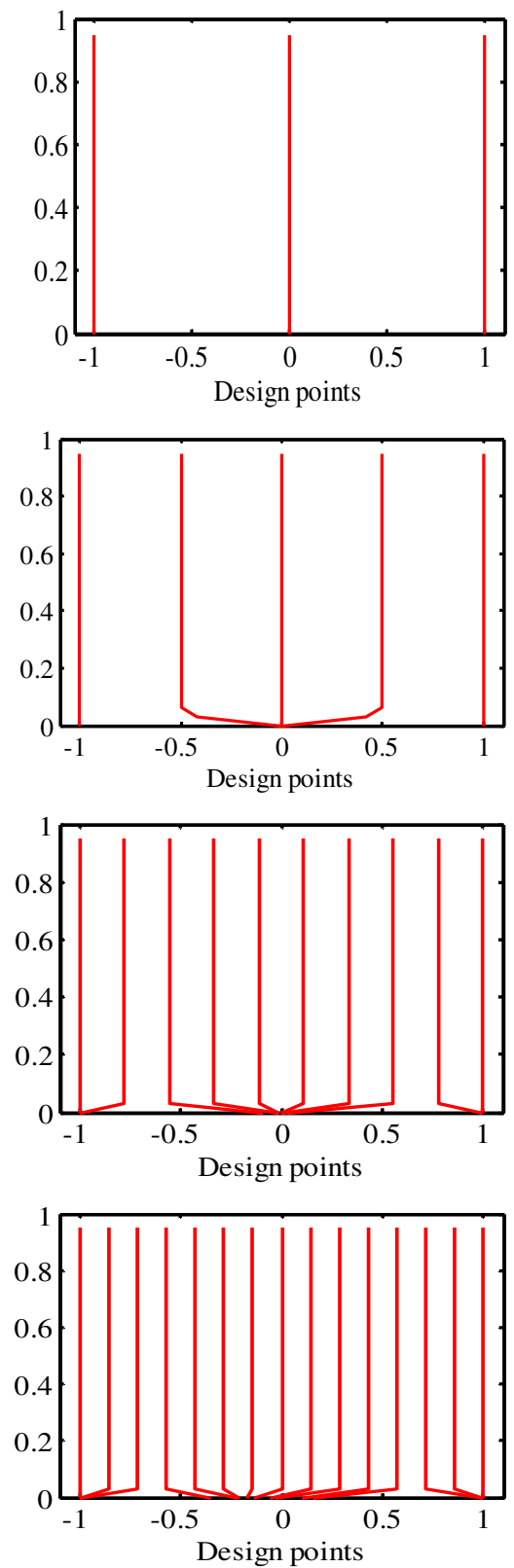

cubic $(p=4)$
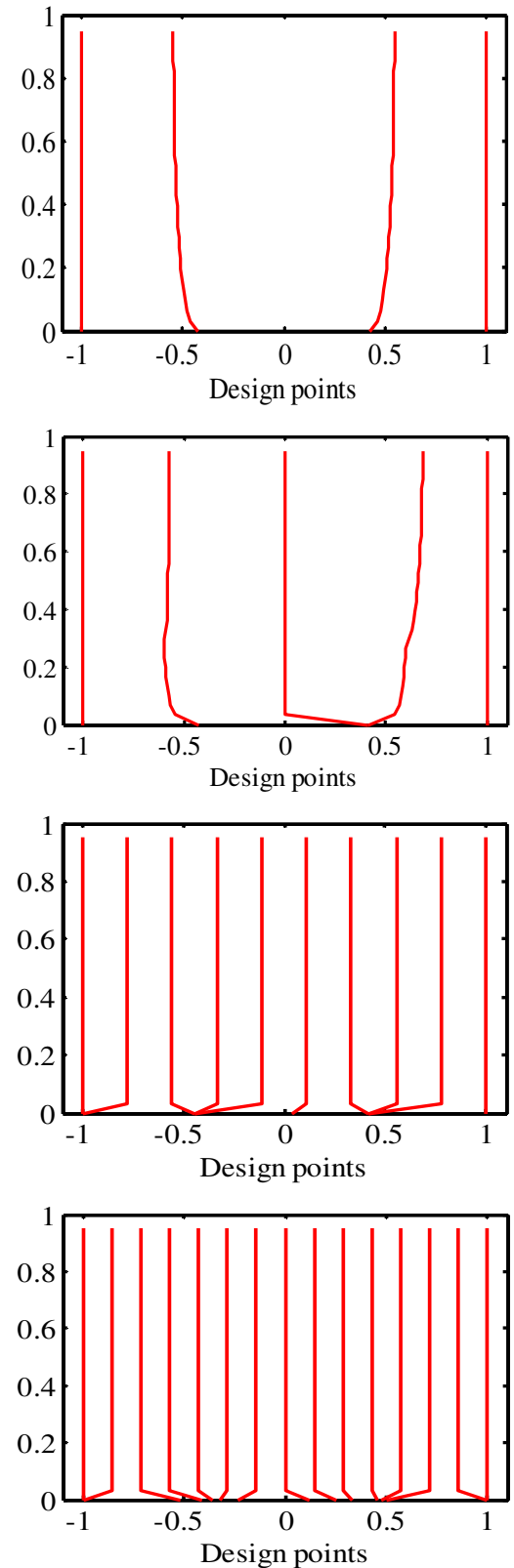

Figure 2.1. Optimal time allocations of time points for polynomial models with $q$ time points. 


\subsubsection{Relative efficiency}

Figures 2.2-2.4 present the RE for different values of cost ratio $k=c_{1} / c_{2}$ and mixed effects models with four different covariance matrices $\mathbf{G}$ of the random parameters, for number of time points $q=5,10$, and 15 , respectively.

Each column in Figures 2.2-2.4 represents the RE corresponding to polynomial models with $p=2,3$, and 4 , respectively, and each row represents a different covariance matrix $\mathbf{G}$. For example, the upper left plot of Figure 2.2 corresponds to the RE of a fixed linear model with $p=2$ parameters, with covariance matrix $\mathbf{G}_{1}=\mathbf{0}$. In each plot, the horizontal axis corresponds to the autocorrelation parameter $\rho$ values and the vertical axis corresponds to the RE. The separate curves in the plots represent the RE for different cost ratios $k=1,2,5,10$ and 20 . The relative efficiencies decrease in general as the serial correlation $\rho$ increases.

Tan and Berger (1999) have shown that for $k=1$ the relative efficiency decreases to $p / q$ as $\rho \rightarrow 1$ for fixed effects models. As follows from Figures 2.2-2.4, this also applies to mixed effects models with random intercept and random slope (see the drawn black lines for $\mathbf{G}_{2}, \mathbf{G}_{3}$ and $\mathbf{G}_{4}$ with $k=1$ ). In general, a design with $p$ time points is the most efficient design when the costs of recruiting a new subject is the same as the costs of an additional observation per subject, i.e. for $k=1$, the $\mathrm{RE}<1$.

A comparison between fixed effects and mixed effects models shows that the relative efficiency decreases when the random effects terms are introduced to the model. This implies that relatively more subjects are needed for mixed effects models compared to the fixed effects models to achieve the same efficiency.

Figures 2.2-2.4 show that the RE increases as the cost ratio $k$ increases. As the costs of a new subject gets higher as compared to the costs of an additional observation per subject, a design with more repeated measurements will gain efficiency. As is stated in the appendix, the $\mathrm{RE} \geq 1$ when the cost ratio $k \rightarrow \infty$ and $\rho \rightarrow 0$, and $\mathrm{RE}=1$ when the cost ratio $k \rightarrow \infty$ and $\rho \rightarrow 1$. 

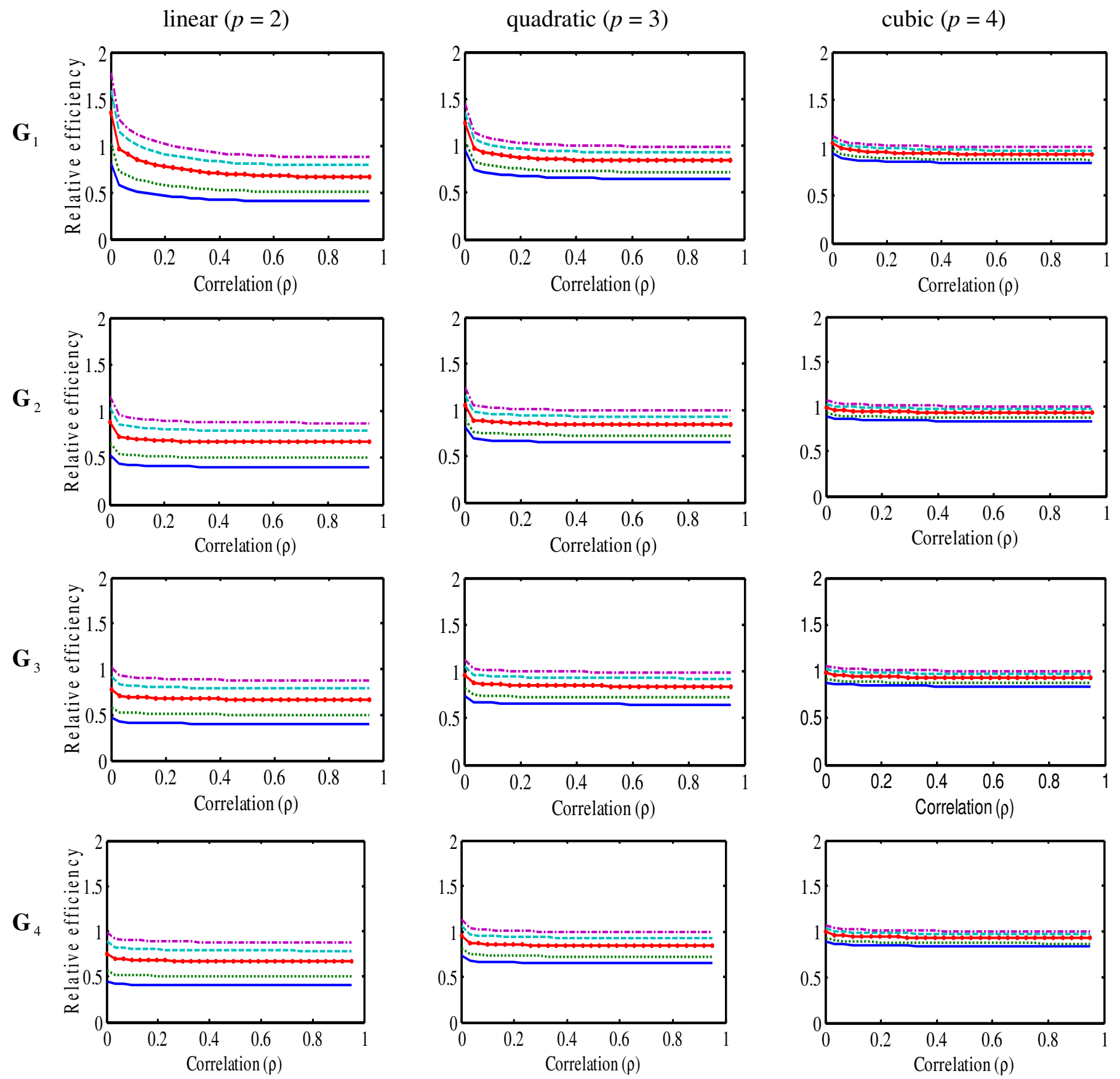

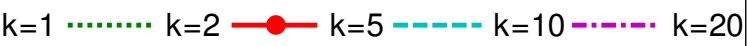

Figure 2.2. RE of designs with $q=5$ time points compared to $q=p$ time points for different cost ratios, $k$. 

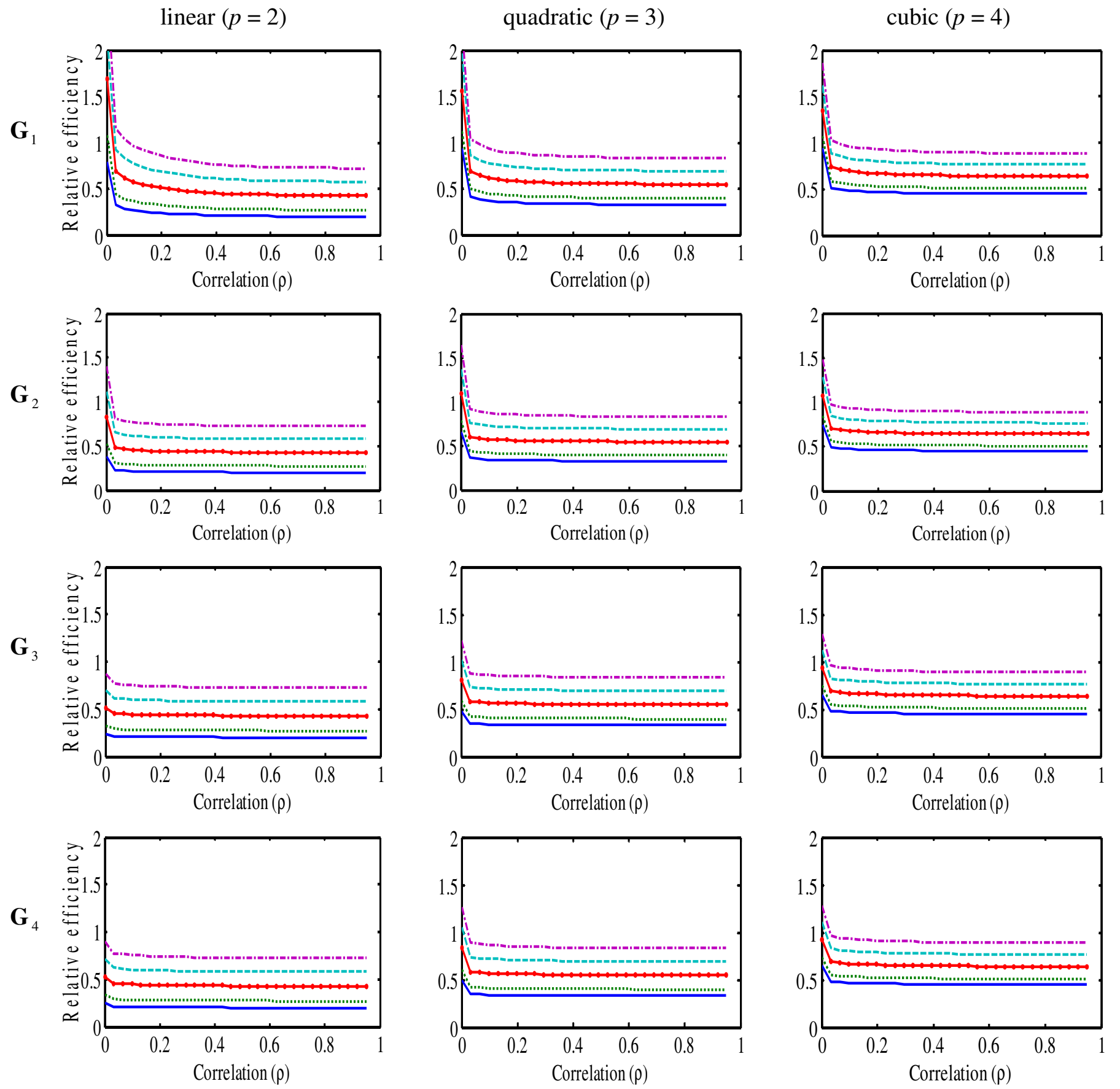

$$
\longrightarrow \mathrm{k}=1 \ldots \ldots \ldots \mathrm{k}=2 \longrightarrow \mathrm{k}=5-\cdots \mathrm{k}=10-\cdots \mathrm{k}=20
$$

Figure 2.3. RE of designs with $q=10$ time points compared to $q=p$ time points for different cost ratios, $k$. 

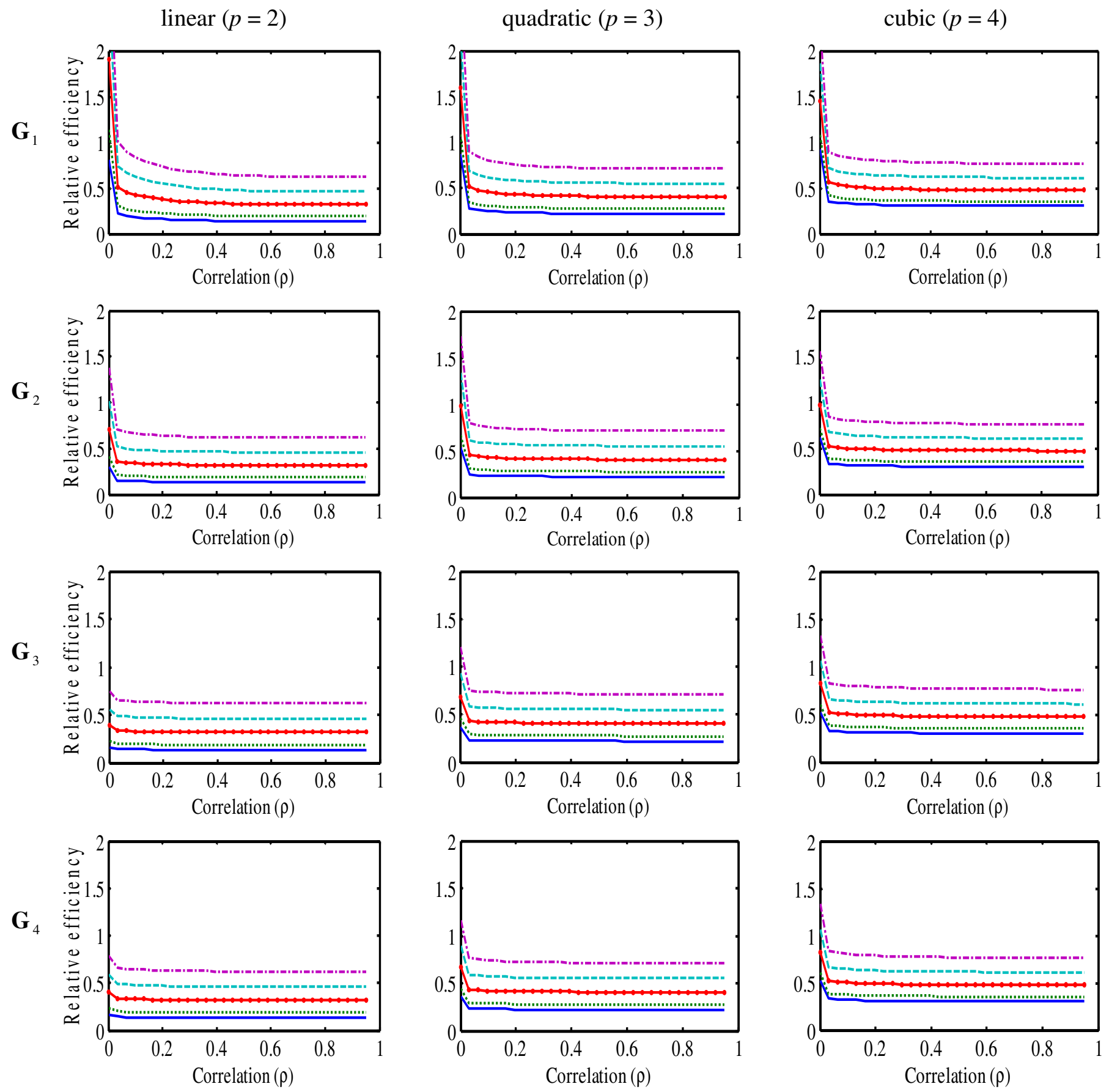

$$
\longrightarrow \mathrm{k}=1 \ldots \ldots \ldots \mathrm{k}=2 \longrightarrow \mathrm{k}=5-\cdots+-\mathrm{k}=10-\cdots \cdot \mathrm{k}=20
$$

Figure 2.4. RE of $q=15$ time points compared to $q=p$ time points with different cost ratios, $k$. 


\subsection{An application}

The growth data introduced by Potthoff and Roy (1964) will be used in this section to illustrate the advantages of optimal designs. These data, presented by Verbeke and Molenberghs (2000) among others, contain growth measurements for 11 girls and 16 boys. For each subject, the distance from the center of the pituitary to the maxillary fissure (head circumference) was recorded at ages $8,10,12$, and 14. The profile plot of each subject is presented in Figure 2.5. Verbeke and Molenberghs (2000) have done extensive analysis on these data and found that a random intercept model without serial correlation can best summarize the head circumferences over time.
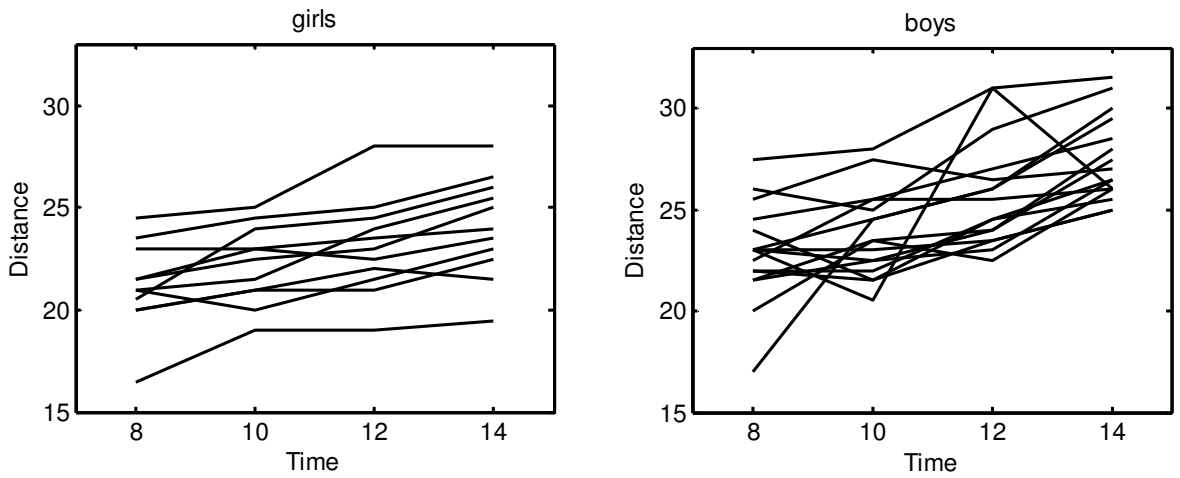

Figure 2.5. Profile plots for the growth data example.

We have computed the RE of the original design compared to the D-optimal design for the number of time points $q$ equal to the number of regression parameters in the linear random intercept model $(p=2)$ with the assumption of $\operatorname{AR}(1)$ correlation structure. No information is given about the costs of the original study. Therefore, we have assumed different values of the cost ratio $k=c_{1} / c_{2}=1,2,5,10$ and 20. The RE values are given in Figure 2.6 for different values of the cost ratios, $k$. 


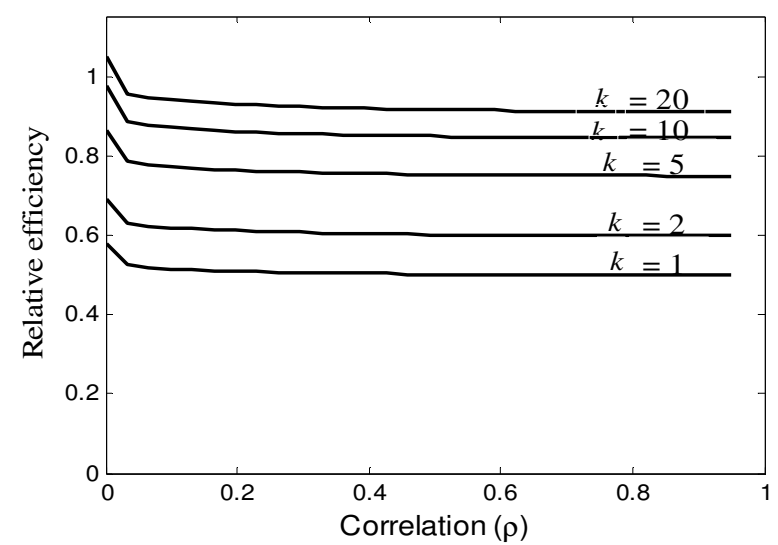

Figure 2.6. RE of the original design at 4 time points for growth data compared to an optimal design with $\mathrm{q}=\mathrm{p}=2$ time points.

Each line in Figure 2.6 represents the RE for a given cost ratio, $k$. In all cases, the RE < 1 which implies that the original design is less efficient as compared to the optimal design. From Figure 2.6, the RE of the observed design is close to 1 when there is no correlation between measurements per subject and $k=c_{1} / c_{2}=20$. When the cost ratio $k<20$, the $\mathrm{RE}$ becomes smaller than 0.90. Especially, when the cost ratio $k=1$, the $\mathrm{RE}$ is about 0.50 which implies that the observed design needs $1 / 0.50=2$ times more subjects in each group of subjects to have the same efficiency for the parameter estimators as the optimal design with 2 time points. Hence, under the assumption of $k=1$, the total costs can be reduced by $1 / 2$ by using the optimal design. The optimal design will save resources in terms of money and time.

\subsection{Discussion and conclusion}

Researchers of repeated measurements studies often face the problem of an adequate selection and allocation of the repeated measurements in time. Usually the number of repeated measurements and the selection of the time points at which the measurements are taken are done on a more or less ad-hoc basis and too many repeated measurements are taken. It is important to consider the costs of the study in identifying efficient designs for longitudinal studies. In addition to the condition of the costs parameters, the efficiency of a design also depends on the number of fixed effect parameters in the model.

When adding a new subject is as expensive as adding an observation per subject, an optimal design with the number of time points $(q)$ equal to the number of fixed effects 
parameters $(p)$ with optimal allocations of time points is the most efficient design. Using more time points $q>p$ leads to a minimum efficiency equal to $p / q$. However; when the costs of adding a new subject is much higher than the costs of adding an additional observation per subject, a design with a number of time points $q>p$ can be as efficient as a design with $p$ time points if the autocorrelation between successive responses is close to 1. If on the other hand, the autocorrelation is close to 0 , then a design with a number of time points $q>p$ can be more efficient than a design with $p$ time points. However, the superiority of a design with more than $p$ time points in terms of efficiency is generally not large in the latter case. Thus, an optimal design with $p=q$ time points can be recommended in general for longitudinal studies. There is also a benefit in terms of probability of drop-out when only a few repeated measurements are planned.

Usually a researcher will have a fairly good notion of the degree of a polynomial that will fit the data best. In case of doubt about the degree of the polynomial, whether it is linear, quadratic, cubic or quartic model, an optimal design with 5 time points could be considered and the fit of the model can be checked. As is shown in Figure 2.2, the RE of this design compared to the optimal designs with $p$ time points is high in most cases. Alternatively, pilot studies may provide accurate information about the order of the polynomial.

This article focuses on the optimal designs for the estimation of parameters which describe the change over time within one group. The results could be extended for the purpose of comparing two or more groups over time. Although we expect the results not to be very different in these cases, further study will be needed to confirm this conjecture. We only used a linear cost function in this study, because this cost function is expected to apply to most cohort studies. It is assumed in this thesis that all subjects are available for the study over the study period and there are no dropouts. Although it needs more research, a recent study concluded that if one uses a D-optimal design based on complete data and dropout is encountered during data collection stage, the loss in efficiency doe not exceed $15 \%$. Thus, one can compensate for the small loss by increasing the initial sample size by at most 15\% (Ortega-Azurdy et al., 2007). 
Appendix: Properties of RE at $\rho=0$ and $\rho=1$ when the cost ratio $k=c_{1} / c_{2} \rightarrow \infty$ From equations (2.5):

$$
\lim _{k \rightarrow \infty} \operatorname{RE}\left(\xi_{q}^{*}, \xi_{p}^{*}\right)=\left\{\frac{\operatorname{det}\left\{\left(\mathbf{X}_{(p)}^{\prime}\left(\mathbf{Z}_{(p)} \mathbf{G} \mathbf{Z}_{(p)}^{\prime}+\sigma^{2} \mathbf{R}_{(p)}\right)^{-1} \mathbf{X}_{(p)}\right)^{-1}\right\}}{\operatorname{det}\left\{\left(\mathbf{X}_{(q)}^{\prime}\left(\mathbf{Z}_{(q)} \mathbf{G} \mathbf{Z}_{(q)}^{\prime}+\sigma^{2} \mathbf{R}_{(q)}\right)^{-1} \mathbf{X}_{(q)}\right)^{-1}\right\}}\right\}^{1 / p},
$$

where $\mathbf{X}_{(p)}, \mathbf{Z}_{(p)}$, and $\mathbf{R}_{(p)}$ are the corresponding design and covariance matrices for subjects with $p$ time points; and $\mathbf{X}_{(q)}, \mathbf{Z}_{(q)}$, and $\mathbf{R}_{(q)}$ are accordingly the design and covariance matrices for subjects with $q$ time points.

Based on this result, the following properties are given at the boundaries of $\rho$ :

i) $\lim _{k \rightarrow \infty} \lim _{\rho \rightarrow 1} \operatorname{RE}\left(\xi_{q}^{*}, \xi_{p}^{*}\right)=1$

If the correlation between successive responses approaches 1 , then the addition of a measurement per subject does not affect the amount of information on the parameters. It follows, that close to $\rho=1$ the determinants in the equation become equal and then the RE equal to 1 .

ii) $\lim _{k \rightarrow \infty} \lim _{\rho \rightarrow 0} \operatorname{RE}\left(\xi_{q}^{*}, \xi_{p}^{*}\right) \geq 1$

In the uncorrelated situation with fixed effects, the addition of a measurement per subject is the same as an addition of a new measurement from a new subject. Thus, the design with $q$ time points will have more extra information and the determinant in the denominator of the equation will be greater or equal to the determinant in the numerator. Thus, when $\rho=$ 0 and the model is fixed effects, the RE will be greater or equal to 1 . 



\section{D-optimal cohort designs for linear mixed effects models}

This chapter (in slightly reduced form) is published in Statistics in Medicine, 27:2586-2600 and available online since 29 August 2007 (DOI: 10.1002/sim.3045), Authors Tekle, F.B., Tan, F.E.S., and Berger, M.P.F. 


\begin{abstract}
:
The D-optimality criterion is used to construct optimal designs for different number of independent cohorts, which constitute a number of repeated measurements per subject over time. A cost function for longitudinal data is proposed, and the optimality criterion is optimized taking into account the cost of the study. First, an optimal number of design points for a given number of cohorts and cost were identified. Then, an optimal number of cohorts is identified by comparing relative efficiencies. A numerical study shows that for models describing the trend of a continuous outcome over time by polynomials, the most efficient number of repeated measurements is equal to the sum of the total number of cohorts and the degree of the polynomial in the model. Relative efficiencies of purely longitudinal cohort design with only one cohort, mixed longitudinal and cross-sectional cohort designs with more cohorts are compared. The results show that, a purely longitudinal cohort design with only one cohort of subjects measured at the optimal time points is the most efficient design. The findings in this chapter show that, with only a few repeated measurements one can obtain a highly efficient design for parameter estimation. The results of this study will reduce the cost of data collection and ease the logistical burdens in cohort studies.
\end{abstract}

Key words: D-Optimal design; Cohort design; Relative efficiency; Longitudinal design; Repeated measurements; Cost. 


\subsection{Introduction}

Researchers in health sciences and medicine often are interested in estimating changes over time. For example, if a researcher is interested in describing change of health status over time, then it would be possible to design a study in which health status of a cohort of people is assessed at different time points. In the past, many large-scale longitudinal cohort studies have been set up for this purpose. Some examples of such studies are the Framingham heart study (Dawber, 1980), the national cancer prevention study (Calle et al., 2002), the nurses' health study (Colditz, 1994; Camargo and Willett, 1999), the New York state cohort study (Bandera et al., 1997), the longitudinal aging study of Amsterdam (Dik et al., 2000), and the Survey of Medical Information and Life style study in Eindhoven (SMILE) (Van den Akker et al., 2008).

These large scale studies all have in common that different cohorts of people are measured several times over a long period of time. For example, the national cancer prevention study used 68000 volunteers and measured them over a time span of 12 years (Calle et al., 2002), and the SMILE project choose 35000 patients from different general practitioners offices and plans to measure them every year over 10-15 years. These studies also encounter irregular drop-out and are very expensive. The costs of these large scale studies may be reduced by choosing the design more carefully and by selecting samples and cohorts more efficiently. Moreover, the number of repeated measures may also be selected to optimize the information. It may therefore be worthwhile to apply optimal design procedures to design these large scale studies.

Depending on the number of cohorts in the design, the design may be purely longitudinal, with only one cohort measured over the complete time interval, mixed longitudinal design, with several cohorts measured over a shorter time interval for each cohort and cross-sectional, where groups of subjects are measured at a single time point for each group (Rao and Rao, 1966; Woolson et al., 1978).

In this chapter, we will consider the optimal cohort designs for a regression model that relates the expected values of a continuous outcome variable with a polynomial function of time of at most degree 3. Berger (1986) compared the efficiencies of the longitudinal, mixed longitudinal and cross-sectional designs and found that the degree of the polynomial of the fixed effects model is crucial for the selection of the most efficient design. Using equidistance time points, Berger (1986) found that a longitudinal cohort design is the most efficient one when the degree of the polynomial approaches the number of repeated 
measurements, and that the cross sectional design is the most efficient one when a linear function is assumed and the correlations between repeated measurements are not too large. Rao and Rao (1966) compared the standard errors of the growth rate estimates obtained from a mixed longitudinal design with those obtained from a cross-sectional design in their study of growth rates of height and body weight of Indian school boys. The comparison revealed that the mixed longitudinal design was more efficient than the cross-sectional design. A similar result was found by Woolson et al. (1978). Tan and Berger (1999) gave an optimal number of repeated measurements and allocations for longitudinal studies with one cohort. Ouwens et al. (2002) also gave D-optimal designs for some over-lapping cohort designs.

Three extensions of previous works will be made. The first extension is that a general linear mixed effects model will be considered to describe data of cohort studies and an optimal design approach will be used to find the optimal number of repeated measurements and allocations to maximize the information for the estimation of the parameters in the model. The second extension is that more than one non-overlapping cohorts (independent groups of subjects) for the linear mixed effects model will be considered, i.e., in addition to longitudinal studies, the mixed longitudinal and cross-sectional studies will also be considered. The third extension is to consider cost constraints in the optimization problem by proposing a cost function for cohort designs.

\subsection{Optimal design problems for cohort studies}

The three typical cohort designs for cohort studies are shown in Figure 3.1. The purely longitudinal cohort design in Figure 3.1 has subjects within a cohort measured over the complete time interval. The mixed longitudinal cohort design has three cohorts of subjects measured at three different time intervals, and the cross-sectional cohort design has three groups of subjects each of them measured at one time point within the time interval of the study. As it can be seen from Figure 3.1, a cohort design has data missing by design, that is, it has complete blocks of missing data.

These designs can be distinguished by the following notations. Let cohort $s$ consist of $n_{s}$ subjects that are measured at $q_{s}$ time points $\mathbf{t}_{s}=\left(t_{s 1}, \cdots, t_{s q_{s}}\right)^{\prime}$ in the interval $T_{s}$ for $s=1, \cdots, S$ with the total number of subjects $N=\sum_{s=1}^{S} n_{s}$, and total number of repeated 
measurements $q=\sum_{s=1}^{S} q_{s}$, where $S$ is the total number of cohorts for the study. If the time intervals $T_{s}$ completely overlap, then all subjects can be considered as members of one cohort and $S$ reduces to one. It may be possible to make the cohorts overlap without having a complete overlap. However, for simplicity, we will assume non-overlapping time interval $T_{s}$ for separate cohorts, i.e., $T_{s} \cap T_{s^{\prime}}=\varnothing$, for $s \neq s^{\prime}=1, \cdots, S$.

For a mixed longitudinal cohort design with number of cohorts $S>1$, the $n_{s}$ subjects are measured at $q_{s} \geq 1$ time points $\mathbf{t}_{s}=\left(t_{s 1}, \cdots, t_{s q_{s}}\right)^{\prime} \in T_{s}$. A purely longitudinal design is a special case of a mixed longitudinal cohort design with number of cohorts equal to $S=1$. For a cross-sectional cohort design with number of cohorts equal to the total number of repeated measurements, i.e. $S=q$, the $n_{s}$ subjects are measured at only $q_{s}=1$ time point $\mathbf{t}_{s}=t_{s 1} \in T_{s}$.

The design problem for the cohort studies can be translated into several questions.

- What is the optimal number of cohorts in a cohort design?

$\circ$ What is the optimal number of repeated measurements or time points for each cohort?

- What is the optimal allocation of the time points within a cohort?

In general a cohort design should be chosen in such a way, that the parameters describing the change of time are estimated as efficiently as possible and that the tests of the overall trend over time have maximum power. 


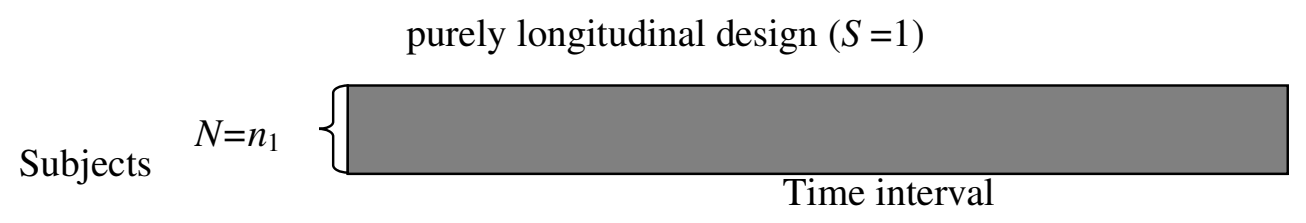

mixed longitudinal design $(S=3)$

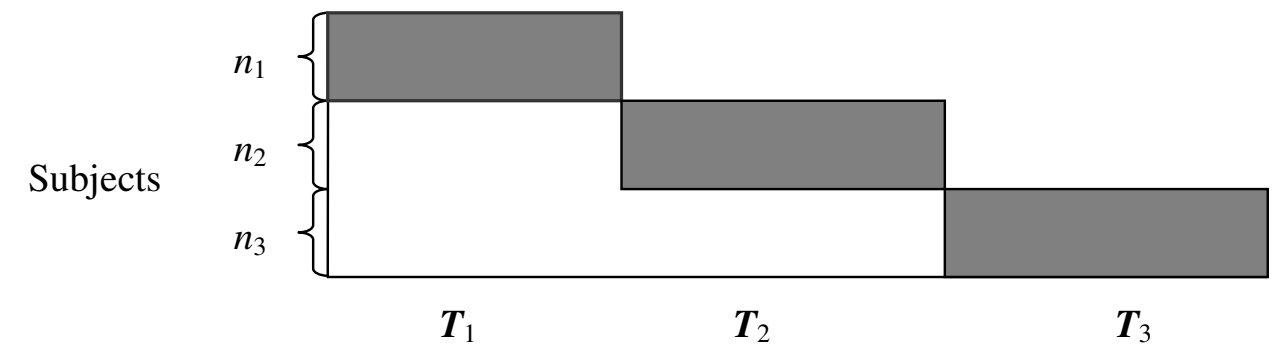

Time interval

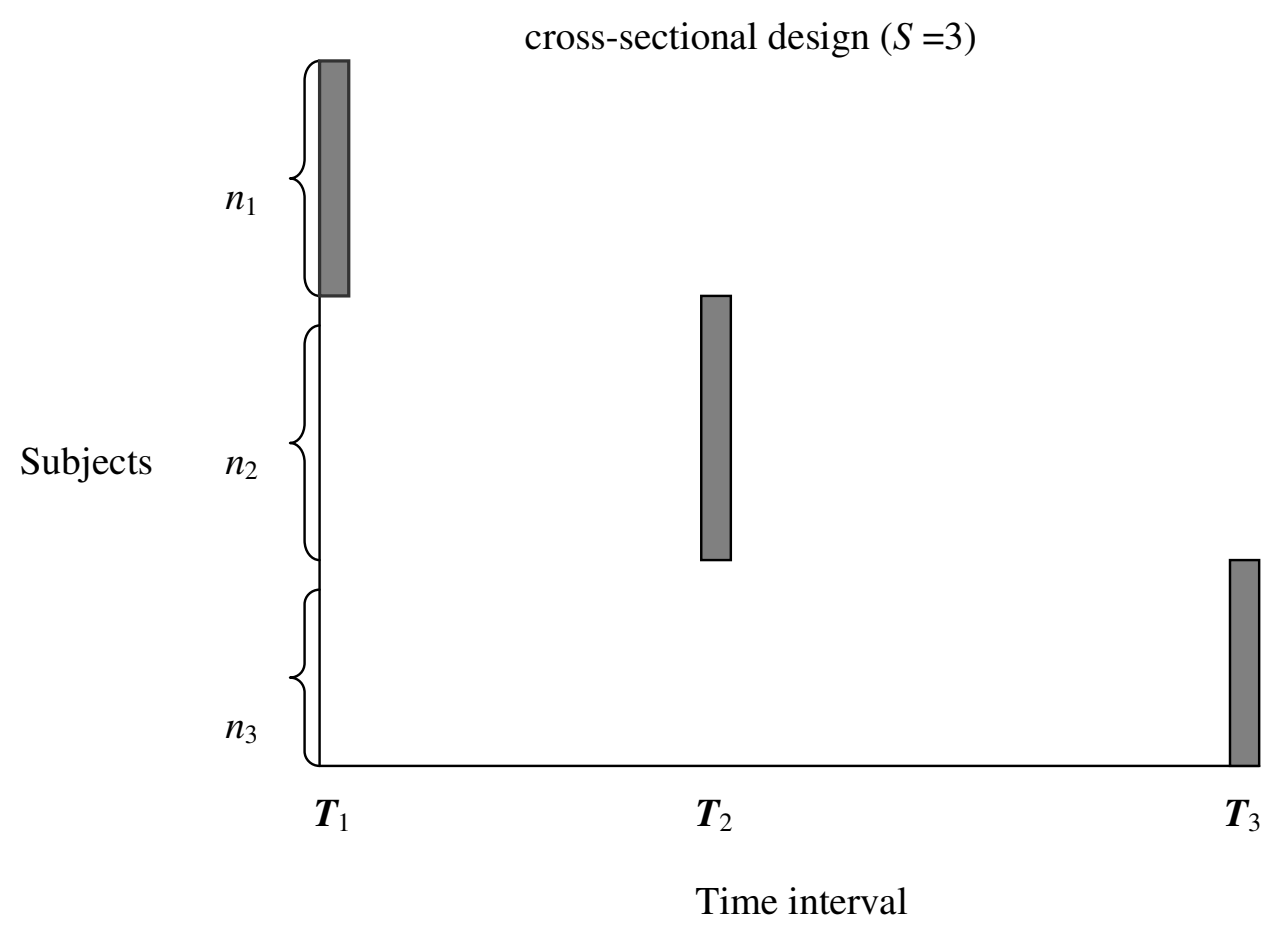

Figure 3.1. Three typical cohort designs.

\subsection{Linear mixed model}

A linear mixed model is proposed and discussed by Diggle (1988), Diggle et al. (1994), Cnaan et al. (1997), and Verbeke and Molenberghs (2000), among others, for the analysis of repeated measures and longitudinal data. The model is general in a sense that it can handle unbalanced repeated measures data obtained in a longitudinal study. As mentioned by Cnaan et al. (1997), there is no requirement for the linear mixed model that all subjects 
have an equal number of repeated measurements, or that subjects are measured at the same time points.

Let $\mathbf{y}_{s i}=\left(y_{s i 1}, \cdots, y_{s i q_{s}}\right)^{\prime}$ be the $q_{s} \times 1$ vector of repeated measurements of a continuous response variable for person $i, i=1, \cdots n_{s}$, at $q_{s}$ time points $\mathbf{t}_{s}=\left(t_{s 1}, \cdots, t_{s q_{s}}\right)^{\prime} \in T_{s}$ in cohort $s, s=1, \cdots, S$. The class of linear regression models with random effects is given by:

$$
\mathbf{y}_{s i}=\mathbf{X}_{s} \boldsymbol{\beta}+\mathbf{Z}_{s} \mathbf{b}_{s i}+\boldsymbol{\varepsilon}_{s i},
$$

where, for each subject $i$ in cohort $s, \mathbf{X}_{s}$ is the $q_{s} \times p$ matrix of explanatory variables and $\mathbf{Z}_{s}$ is the $q_{s} \times r$ submatrix of $\mathbf{X}_{s} ; p$ and $r$ are numbers of fixed and subject specific regression parameters, respectively. The matrices $\mathbf{X}_{s}$ and $\mathbf{Z}_{s}$ consist of polynomial coefficients based on $\mathbf{t}_{s}$. The $p \times 1$ vector $\boldsymbol{\beta}$ is a vector of fixed regression coefficients. The $r \times 1$ vector $\mathbf{b}_{s i}$ is the vector of subject specific coefficients of subject $i$ within cohort $s$, with mean zero and $r \times r$ variance-covariance matrix $\mathbf{G}$. The $q_{s} \times 1$ vector $\boldsymbol{\varepsilon}_{s i}$ is the vector of random errors for subject $i$ within cohort $s$, with mean zero and variancecovariance matrix $\sigma^{2} \mathbf{R}$, where the $q_{s} \times q_{s}$ matrix $\mathbf{R}_{s}$ is the correlation matrix of the error vector and $\sigma^{2}$ is a common variance for error components. Further, it is assumed that the vectors $\mathbf{b}_{s i}$, and $\boldsymbol{\varepsilon}_{s i}$ are independent. Due to the consequence of this last assumption, the variance-covariance matrix of $\mathbf{y}_{s i}$ is given by:

$$
\mathbf{V}_{s}=\mathbf{Z}_{s} \mathbf{G} \mathbf{Z}_{s}^{\prime}+\sigma^{2} \mathbf{R}_{s} .
$$

The matrix $\mathbf{V}_{s}$ is determined by the structure of $\mathbf{R}_{s}$ and by the variance-covariances of the random effects in $\mathbf{G}$.

We will restrict ourselves to a first order autoregressive correlation structure (AR1) with parameter $\rho$ for $\mathbf{R}_{s}$ and three different cases for random effects. The first case has no random effects $\left(\mathbf{Z}_{s}=\mathbf{0}\right)$, the second case has a single subject effect (intercept) where $\mathbf{Z}_{s}$ is a vector of ones, and the third case for random effects has subject specific intercepts and subject specific slopes where $\mathbf{Z}_{s}$ is a matrix whose first column is 1's and the second column contains the vector of time points $\mathbf{t}_{s}$.

To describe the changes over time the $q_{s} \times p$ design matrix $\mathbf{X}_{s}$ contains polynomial coefficients on the vector of time points $\mathbf{t}_{s}$. For example, $\mathbf{X}_{s}$ for cubic coefficients is given by 


$$
\mathbf{X}_{s}=\left[\begin{array}{cccc}
1 & t_{s 1} & t_{s 1}^{2} & t_{s 1}^{3} \\
\vdots & \vdots & \vdots & \vdots \\
1 & t_{s q_{s}} & t_{s q_{s}}^{2} & t_{s q_{s}}^{3}
\end{array}\right]
$$

The best linear unbiased estimator of $\boldsymbol{\beta}$ has variance-covariance matrix (Cnaan et al., 1997; Rao, 1973)

$$
\operatorname{var}(\hat{\boldsymbol{\beta}})=\left[\sum_{s=1}^{S} n_{s}\left\{\mathbf{X}_{s}^{\prime} \mathbf{V}_{s}^{-1} \mathbf{X}_{s}\right\}\right]^{-1},
$$

and the determinant $\operatorname{det}\{\operatorname{var}(\hat{\boldsymbol{\beta}})\}$, which is often referred as the generalized variance, will be used for the definition of the optimal cohort design. First, however, a cost function is presented.

\subsection{Cost consideration}

Taking more measurements per subject obviously provides additional information and increases the precision of parameter estimators. However, the number of measurements per subject usually needs to be restricted because of monetary cost, subject fatigue, and other logistical reasons. Therefore, it is reasonable to take the cost of measuring into account when a cohort design is optimized.

Suppose that the costs of the initial setup of a cohort design is $c_{0}$. This quantity $c_{0}$ may also include the costs of maintaining the research staff during the study period. Let the costs of recruiting a new subject be $c_{1}$, and the costs of obtaining a repeated measurement per subject be $c_{2}$. Then the total costs of the study with $S$ cohorts and $q_{s}$ repeated measurements for $n_{s}$ subjects within a cohort may be represented by the linear cost function

$$
C=c_{0}+\sum_{s=1}^{S} n_{s}\left(c_{1}+c_{2}\left(q_{s}-1\right) .\right.
$$

Note that both $c_{1}$ and $c_{2}$ could vary from cohort to cohort but for simplicity, we will keep them the same in each cohort. In practice, recruiting a subject for a study will often cost more than the cost of obtaining a measurement per subject, i.e., $c_{1} \geq c_{2}$. The cost of maintaining the research could become more expensive with time. In this chapter, however, the cost function in (3.5) assumes a constant cost $c_{0}$ of maintaining the research regardless of the study duration. 


\subsection{D-optimal Cohort Design}

The cohort designs $\xi \in \Xi_{S q}$ are of the form

$$
\xi=\left\{\begin{array}{llll}
\mathbf{t}_{1} & \mathbf{t}_{2} & \cdots & \mathbf{t}_{S} \\
w_{1} & w_{2} & \cdots & w_{S}
\end{array}\right\},
$$

where $\Xi_{S q}$ is the design space of all cohort designs with $S$ cohorts such that the total number of repeated measurements equal to $q=\sum_{s=1}^{S} q_{s}, w_{s}$ is the relative size of cohort $s$ such that $\sum_{s=1}^{S} w_{s}=1$ and $\mathbf{t}_{s}=\left(t_{s 1}, \cdots, t_{s q_{s}}\right)^{\prime} \in T_{s}$.

The cost of measuring a subject depends on the number of repeated measurements, i.e., the cost of measuring a subject with many repeated measurements is generally more than the cost of measuring a subject with relatively few repeated measurements. Thus, the variance-covariance matrix given in (3.4) under the design $\xi$ should be normalized for the cost. Fedorov et al. (2002) proposed to normalize the variance-covariance matrix for each cohort for the total cost of data collection. The normalized variance-covariance matrix is (Fedorov et al., 2002; Gagnon and Leonov, 2005):

$$
\operatorname{var}\left(\hat{\boldsymbol{\beta}}_{\xi}\right)=\left[\sum_{s=1}^{s} w_{s}\left(\frac{\mathbf{X}_{s}^{\prime} \mathbf{V}_{s}^{-1} \mathbf{X}_{s}}{c_{1}+c_{2}\left(q_{s}-1\right)}\right)\right]^{-1},
$$

with $w_{s}=\frac{n_{s}\left(c_{1}+c_{2}\left(q_{s}-1\right)\right)}{C-c_{0}}$. Note that the normalized variance-covariance matrix $\operatorname{var}\left(\hat{\boldsymbol{\beta}}_{\xi}\right)$ is equal to $\left(C-c_{0}\right) \operatorname{var}(\hat{\boldsymbol{\beta}})$.

If the parameters in the variance-covariance matrix $\mathbf{V}_{s}$ and the costs $c_{1}$ and $c_{2}$ are known, then the generalized variance of $\operatorname{var}\left(\hat{\boldsymbol{\beta}}_{\xi}\right)$ in equation (3.7) can be minimized over the design space $\Xi_{S q}$ by choosing a D-optimal cohort design $\xi^{*}$ such that, for each design $\xi \in \Xi_{S q}$,

$$
\operatorname{det}\left\{\operatorname{var}\left(\hat{\boldsymbol{\beta}}_{\xi^{*}}\right)\right\} \leq \operatorname{det}\left\{\operatorname{var}\left(\hat{\boldsymbol{\beta}}_{\xi}\right)\right\},
$$

where $\hat{\boldsymbol{\beta}}_{\xi^{*}}$ and $\hat{\boldsymbol{\beta}}_{\xi}$ are estimators of $\boldsymbol{\beta}$ under $\xi^{*}$ and $\xi$, respectively i.e., an optimal design is a design which for a given total cost has the smallest generalized variance of model parameter estimators.

The optimal design problem for a cohort study is to identify the optimal time locations of the vector $\mathbf{t}_{s}$, the associated optimal weights $w_{s}{ }^{\prime} s$, and the number of repeated 
measurements in each cohort, $q_{s}$. Once the optimal vector of time locations $\mathbf{t}_{s}{ }_{s}^{\prime} s$, the weights $w_{s}^{*}$ 's, and the $q_{s}^{*}$ 's are obtained, the optimal number of subjects within cohort $s$ can be obtained by

$$
n_{s}^{*}=\frac{w_{s}^{*}\left(C-c_{0}\right)}{c_{1}+c_{2}\left(q_{s}^{*}-1\right)},
$$

which in practice can be rounded to the nearest integer ((Fedorov et al., 2002; Gagnon and Leonov, 2005).

For a purely longitudinal cohort design with only one cohort, it follows that the weight of that single cohort will be $w_{1}=1$ and the optimal design problem will reduce to searching for the optimal locations of $\mathbf{t}_{s}$ within the design region. The total number of subjects will then become:

$$
N^{*}=n_{1}^{*}=\frac{C-c_{0}}{c_{1}+c_{2}\left(q_{1}^{*}-1\right)},
$$

where $q_{1}^{*}$ is the optimal number of repeated measurements for the purely longitudinal cohort design.

For a cross-sectional design with the number of cohorts equal to the total number of measurements, and with one measurement per subject in each cohort, the optimal number of subjects within cohort $s$ will become:

$$
n_{s}^{*}=\frac{w_{s}^{*}\left(C-c_{0}\right)}{c_{1}} .
$$

To compare the efficiencies of two designs $\xi_{1}$ and $\xi_{2}$, the relative efficiency (RE) can be used. The RE of a design $\xi_{2}$ compared to a reference design $\xi_{1}$ is given by the ratio (Atkinson and Donev, 1996):

$$
R E_{\xi_{2} \xi_{1}}=\left[\frac{\operatorname{det}\left\{\operatorname{var}\left(\hat{\boldsymbol{\beta}}_{\xi_{1}}\right)\right\}}{\operatorname{det}\left\{\operatorname{var}\left(\hat{\boldsymbol{\beta}}_{\xi_{2}}\right)\right\}}\right]^{1 / p} .
$$

If the ratio is greater than 1 , then $\xi_{2}$ is more efficient than $\xi_{1}$ and is to be preferred compared to the reference design $\xi_{1}$. The ratio of the determinants between bracket is raised to the power $1 / p$ so that the relative efficiency can be interpreted in terms of the sample size: for example, twice of the number of observations in design $\xi_{2}$ are needed in design $\xi_{1}$ to be as efficient as design $\xi_{2}$, when $R E_{\xi_{2} \mid \xi_{1}}=2$. 


\subsection{Numerical study and results}

The purpose of this numerical study is to find the most efficient cohort design which minimizes the determinant of the normalized variance-covariance matrix of the parameter estimators given the required parameter values for the optimization procedure. The optimal weights are derived numerically for each possible combination of time points using the Broyden-Fletcher-Goldfarb and Shanno (BFGS) algorithm (Fletcher, 1987) and then the derived designs are evaluated based on their relative efficiencies. The optimization and computation procedure used in this numerical study is described in the Appendix. Matlab ${ }^{\odot}$ codes are available from the authors upon request.

\section{Time interval and the order of the polynomial}

The referred large scale cohort studies often measure the subjects over more than 10 to 30 time points. We will assume for our numerical comparison that the maximum number of time point is $q=15$, i.e., the set of time points is $\{1,2, \cdots, 15\}$. From findings of Berger [10], among others, the order of the polynomial is crucial in identifying the most optimal design. Mixed effects models with linear $(p=2)$, quadratic $(p=3)$, and cubic $(p=4)$ polynomials, which are common in practice, will be considered in the numerical study.

\section{Parameter values for elements of variance-covariance matrix $\mathbf{V}_{s}$}

The efficiency of a design depends on the elements of the variance-covariance matrix $\mathbf{V}_{s}$. Note, that the variance-covariance matrix $\mathbf{V}_{s}$ given in equation (3.2) is determined by the structure of $\mathbf{G}$, and by the covariance matrix $\sigma^{2} \mathbf{R}_{s}$. We will consider the following cases for $\mathbf{G}$ :

1) $\mathbf{G}_{1}=\mathbf{0}$, which implies that there are no random effects. The model in equation (3.1) reduces to a fixed effect model with autocorrelated errors.

2) $\mathbf{G}_{2}=\left[\begin{array}{ll}1 & 0 \\ 0 & 0\end{array}\right]$, i.e., a random intercept model with variance of the random intercept $g_{11}$, which can be set to $g_{11}=1$ without loss of generality. 
3) $\mathbf{G}_{3}=\left[\begin{array}{cc}1 & 0 \\ 0 & 0.25\end{array}\right]$, a random intercept and random slope model with variances for the random intercept $g_{11}=1$ and random slope $g_{22}=0.25$. The correlation between random intercept and random slope is assumed zero.

4) $\mathbf{G}_{4}=\left[\begin{array}{ll}1 & 0.5 \sqrt{0.25} \\ 0.5 \sqrt{0.25} & 0.25\end{array}\right]$, a random intercept and random slope model with correlation between the random intercept and random slope $r_{12}=0.5$. Note that the covariance between random intercept and random slope $g_{12}=r_{12} \sqrt{g_{11} g_{22}}$.

A literature scan on longitudinal studies shows that the variance of the random errors $\sigma^{2}$ is often less than the variance of the random intercept $g_{11}$ in many studies. We have chosen $\sigma^{2}=0.25$ in this numerical study. Since time structured data are likely to be positively correlated, the autocorrelation $\rho$ in $\mathbf{R}_{s}$ is chosen to range from 0.0 to 0.9 .

\section{Cost function parameters}

The total cost of the study $C$, and the initial setting up cost $c_{0}$ are arbitrarily fixed at $\$ 100,000$ and $\$ 10,000$, respectively. Note that the values of $C$ and $c_{0}$ will not affect the optimization results. However, $\mathrm{C}$ should be large as compared to $c_{0}$ because studies usually have many subjects in each cohort. The relative size of $c_{1}$ compared to $c_{2}$ is given by $c_{1}=k c_{2}$, and we will consider cases when $k=1$ and $k=10$. This means that the cost of recruiting a subject is equal to the cost of one measurement per subject when $k=1$ and the cost of recruiting a subject is ten times more expensive than the cost of one measurement per subject when $k=10$.

\section{Results}

The main results of the numerical study are summarized as follows. The first summary results are shown in Figures 3.2-3.7 where the relative efficiencies of the designs with the optimal allocation of $q$ time points for a given number of cohorts are compared to a design with $q=15$ time points. Note that the number of time points $q=15$ is arbitrarily chosen for the reference design to compare with other designs with the number of time points $q<15$. The conclusions given in this chapter will not change if other numbers of time points are considered for the reference design. The second summary results are shown in Figure 3.8 
and Table 3.1, where the D-optimal time points and weights are given for a selected case. The third summary results are shown in Figures 3.9-3.10 where the efficiencies of cohort designs given the most efficient number of repeated measurements for each cohort design are compared. We will discuss these results briefly:

\section{i) Optimal number of repeated measurements}

Figures 3.2-3.4 show the relative efficiency (RE) plots of $M$ optimal time points compared to a design with $q=15$ time points for linear $(p=2)$, quadratic $(p=3)$, and cubic $(p=4)$ polynomial models, respectively. The costs $c_{1}$ and $\mathrm{c}_{2}$ are assumed to be equal, i.e., $k=1$. Figures 3.5-3.7 show similar relative efficiency plots for $c_{1}=10 c_{2}$. In these figures, the number of cohorts vary across the columns and the covariance matrix $G$ varies across the rows, while the number of repeated measurements vary within each plot. Since the relative efficiencies are computed with respect to a reference design with 15 time points, these relative efficiencies can be used to compare the designs with different number of time points. As mentioned in section 3.5, a design with large RE is most efficient and preferable. Different markers are used and shown in the legend to make distinction between the relative efficiency lines representing the RE of a design with different number of time points $q$ compared to the design with 15 time points.

It can be seen from Figures 3.2-3.7 that a design with $q=15$ is clearly very inefficient and the choice of the most efficient number of repeated measurements $q *$ depends on the order of the polynomial $p-1$, and on the number of cohorts $S$. In most cases, the optimal number of repeated measurements $q^{*}$ is equal to the sum of the number of cohorts $S$ and the order of the polynomial of the model $(p-1)$, i.e., $q^{*}=S+(p-1)$ : for example, the optimal number of repeated measurements for purely longitudinal cohort design with $S=1$ is the same as the number of fixed effect regression parameters $p$.

An exception was found for the $S=2$ cohort design and a fixed effects linear model with autocorrelation $\rho \leq 0.7$, and the costs $c_{1}$ and $\mathrm{c}_{2}$ assumed to be equal. See the plot in row 1 and column 2 of Figure 3.2. The optimal number of repeated measurements for this case is $q^{*}=2$. This means that the cross-sectional cohort design with $S=q=2$ for $\rho \leq 0.7$ and equal costs $c_{1}=\mathrm{c}_{2}$, is more efficient than the mixed longitudinal cohort design. A similar result was found by Machin (1975) and Berger (1986); however, there is no clear explanation for this exceptional case at the moment. 

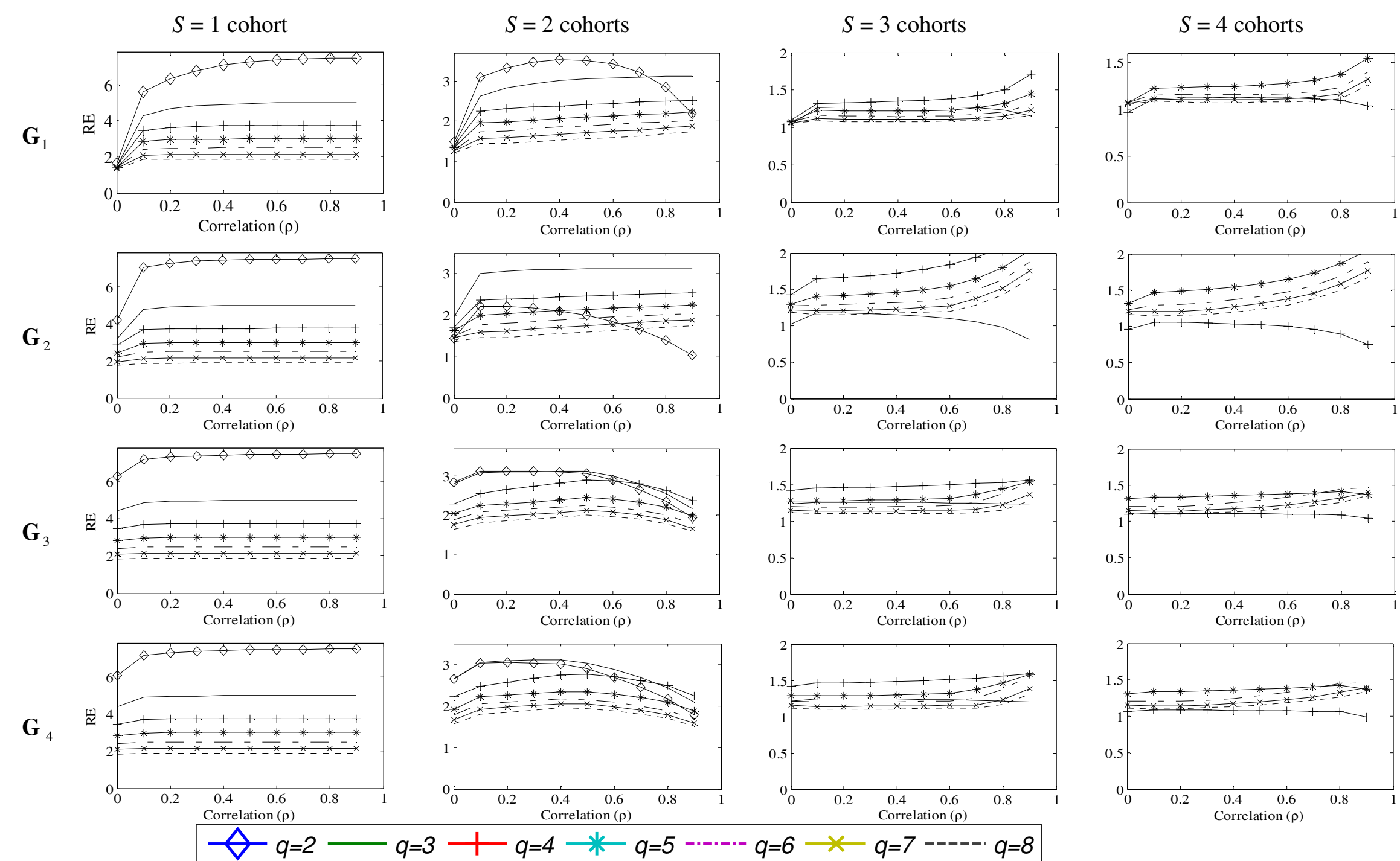

Figure 3.2. RE of optimally allocated $q$ time points compared to $q=15$ time points for a linear model $(p=2)$ with $c_{1}=c_{2}$. 

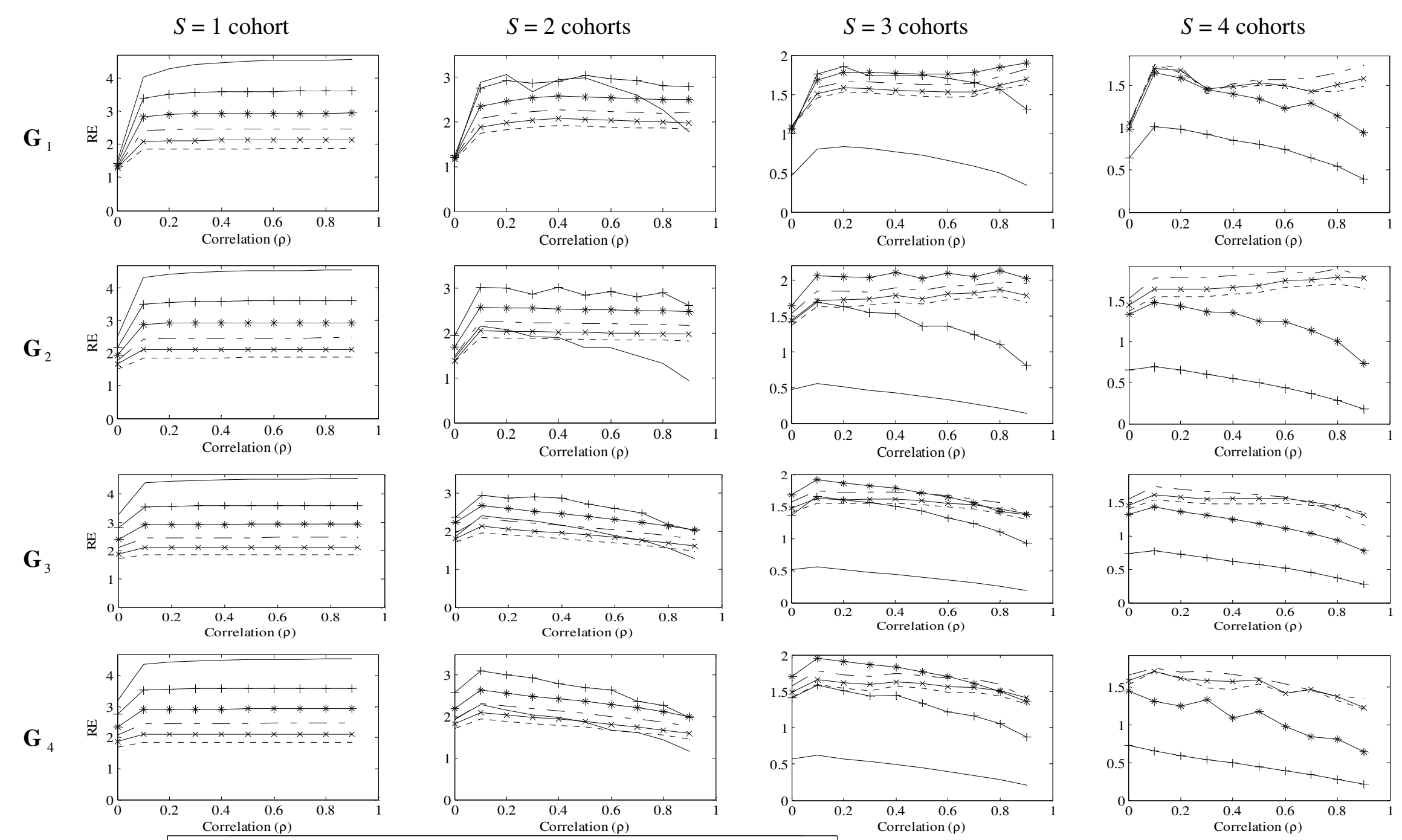

Figure 3.3. RE of optimally allocated $q$ time points compared to $q=15$ time points for a quadratic model $(\mathrm{p}=3)$ with $c_{1}=c_{2}$. 

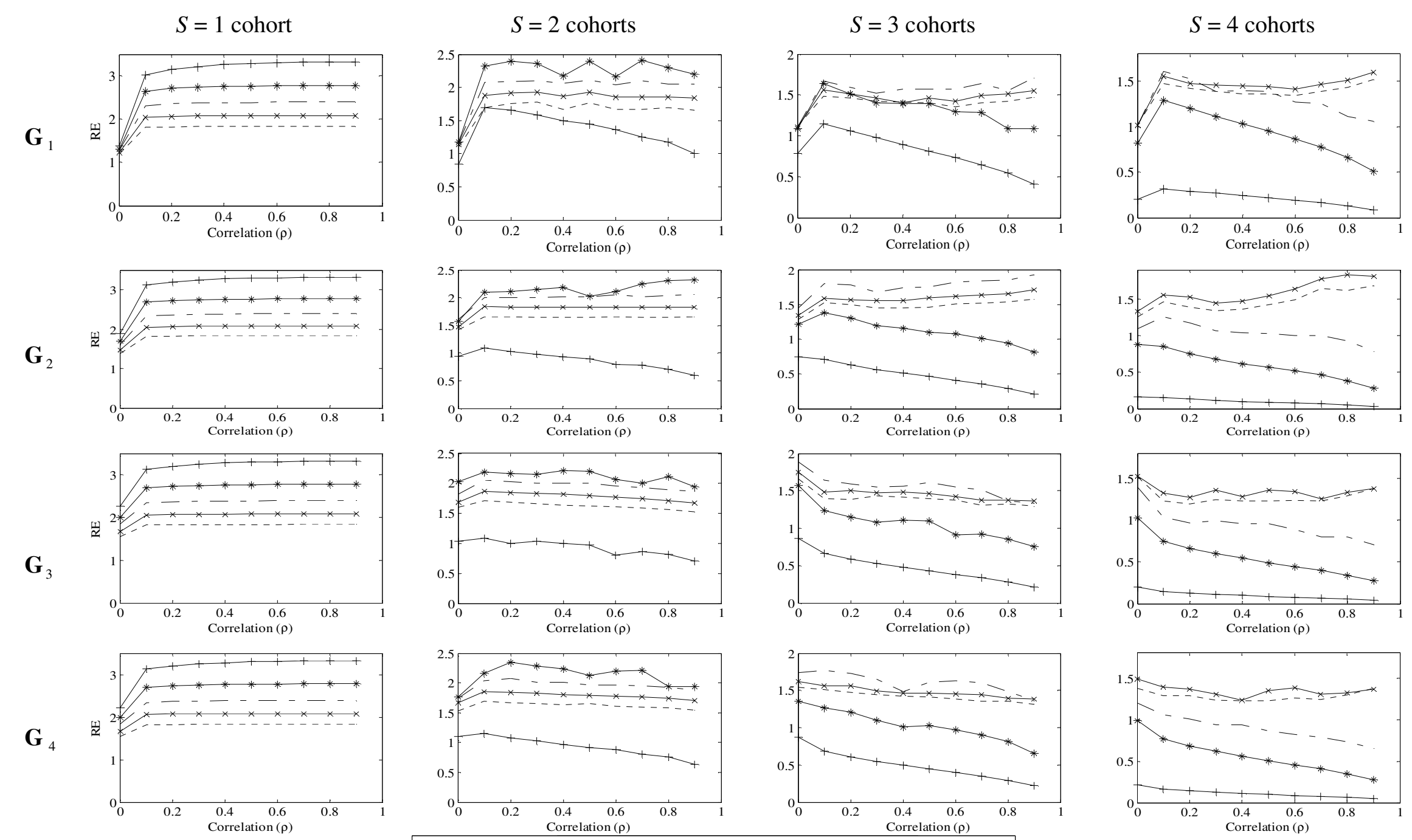

$$
+q=4 \text { * } q=5 \text { - -.-. } q=6 \div q=7 \text {----- } q=8
$$

Figure 3.4. RE of optimally allocated $q$ time points compared to $q=15$ time points for a cubic model $(p=4)$ with $c_{1}=c_{2}$. 

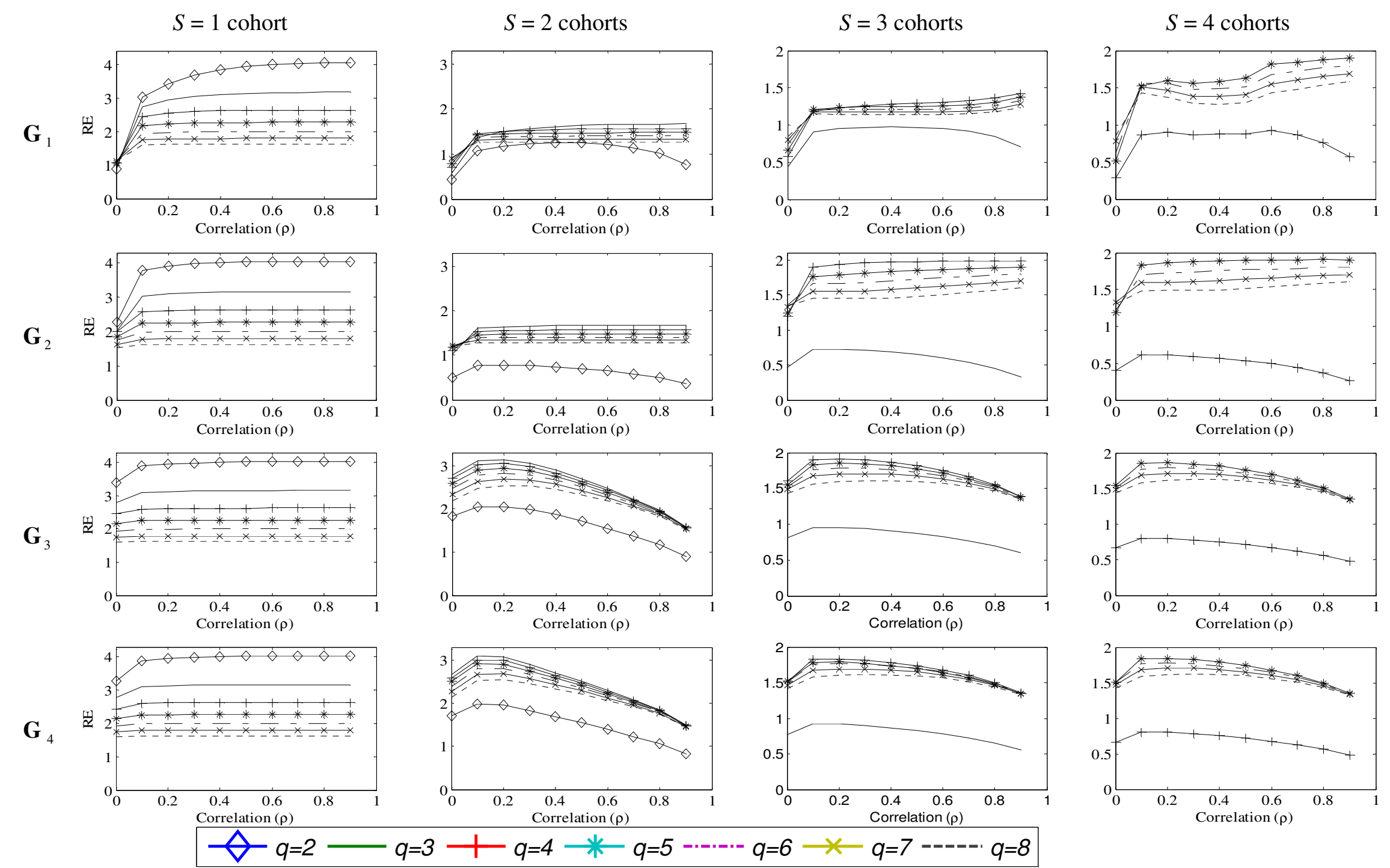

Figure 3.5. RE of optimally allocated $q$ time points compared to $q=15$ time points for a linear model $(p=2)$ with $c_{1}=10 c_{2}$. 

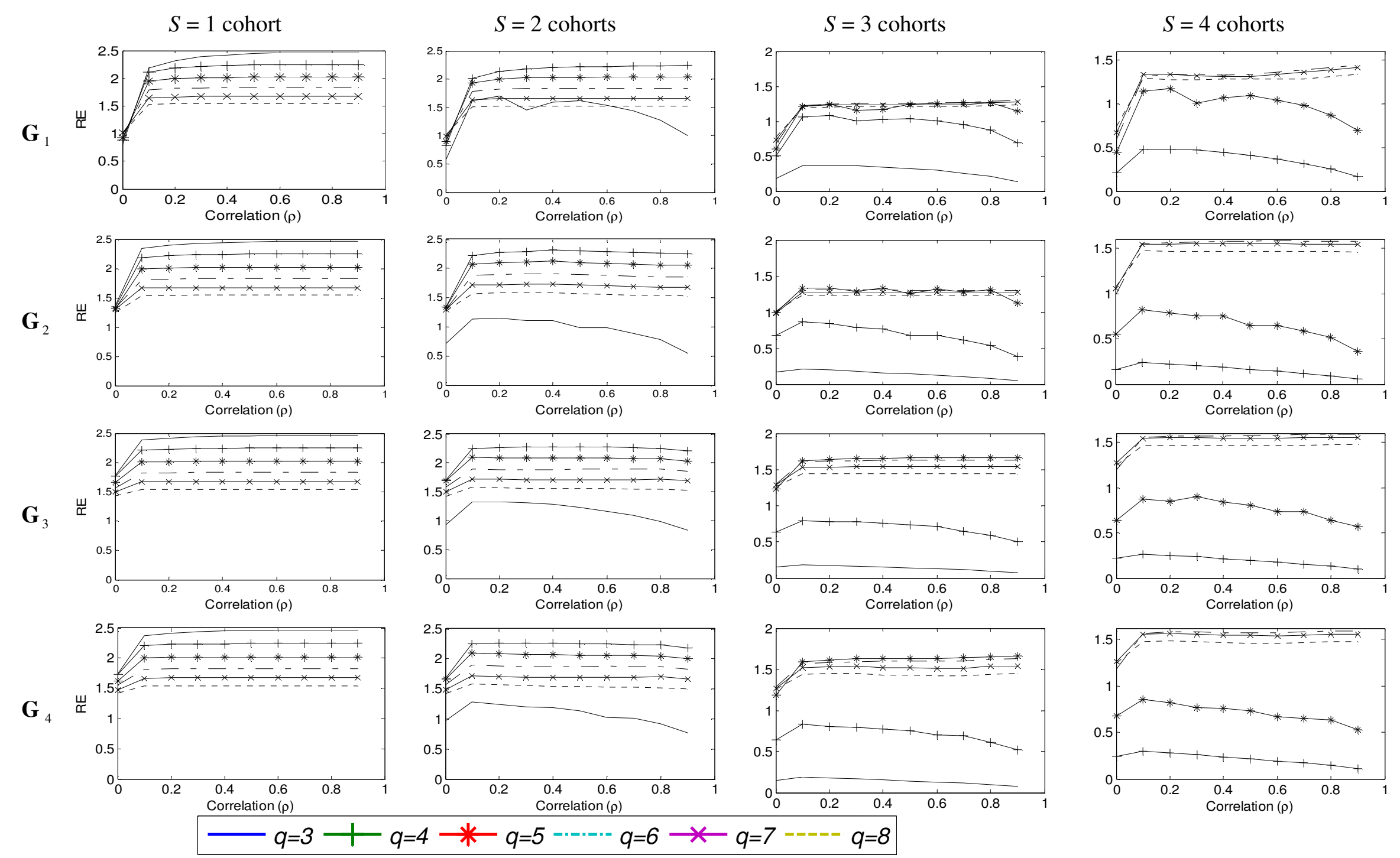

Figure 3.6. RE of optimally allocated $q$ time points compared to $q=15$ time points for a quadratic model $(p=3)$ with $c_{1}=10 c_{2}$. 


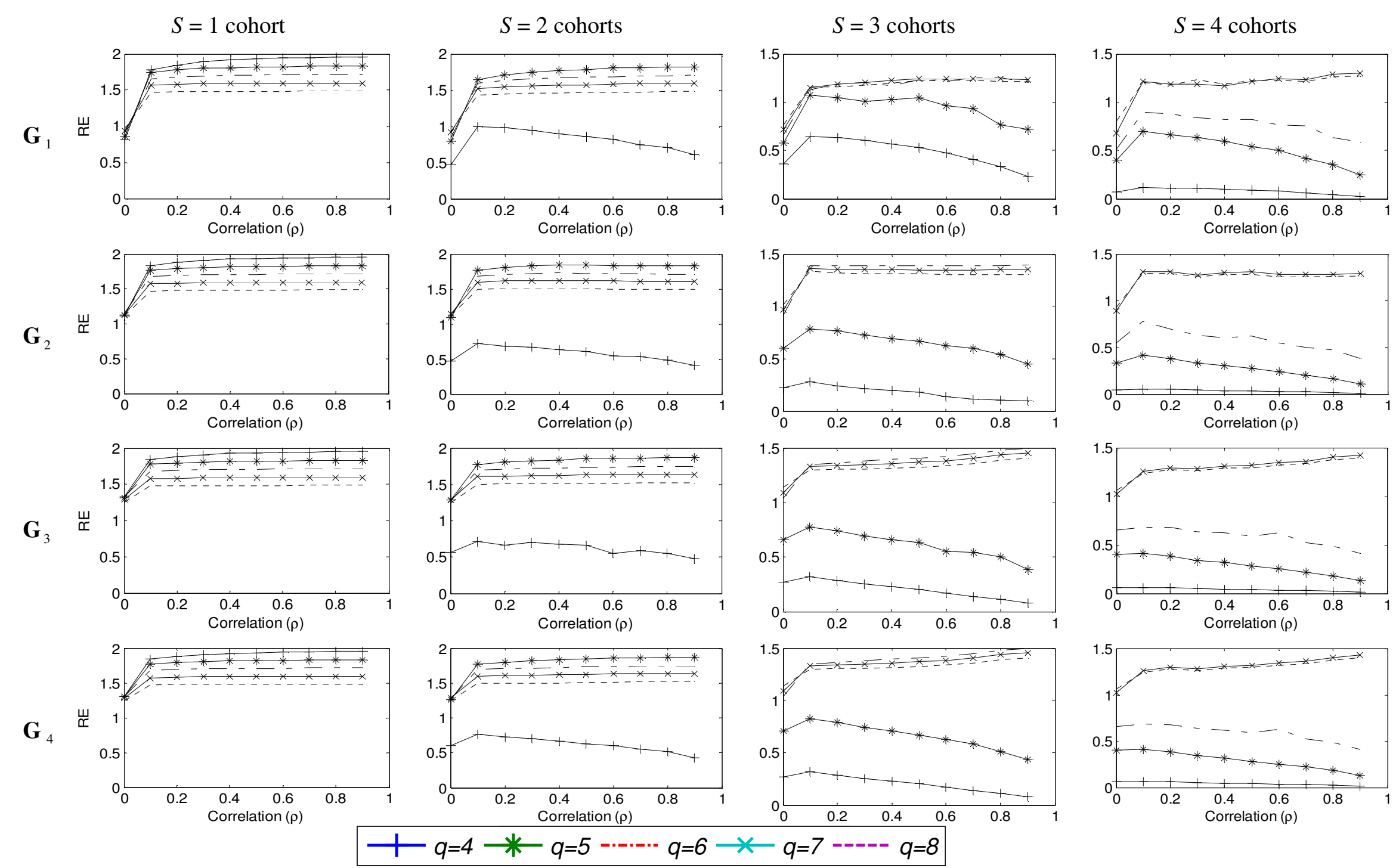

Figure 3.7. RE of optimally allocated $q$ time points compared to $q=15$ time points for a cubic model $(p=4)$ with $c_{1}=10 c_{2}$ 


\section{ii) Optimal allocations of time points}

The optimal allocation of time points for all cohort designs were identified in this numerical study. Actually, the patterns of the allocation of the optimal time points are very similar for all the longitudinal, mixed longitudinal and cross-sectional designs considered in this study.

The optimal allocations of time points for a purely longitudinal cohort design with $S=1$ have been reported by Tan \& Berger (1999). The optimal time points for linear and quadratic models are approximately equally spaced. Although the optimal time points for cubic models are not equally spaced in general, as the number of time points $q$ increases and the serial correlation is not too small $(\rho>0.10)$, they all become approximately equally spaced.

The pattern of the allocation of time points for mixed longitudinal cohort designs with $S>1$ resembles those for the purely longitudinal design. For one of the $S$ cohorts these are $q_{1}^{*}=p \quad$ optimal time points, which are similar to the optimal points for a purely longitudinal design with $S=1$ cohort. The other $(S-1)$ cohorts have $q_{s}^{*}=1$ optimal time point at one of the extreme ends of the time interval. An example of the optimal allocation of time points for the mixed longitudinal cohort design with $S=3$ is given in Figure 3.8. The corresponding optimal weights are given in Table 3.1. It can be seen, that the time point in the third cohort does not have any weight.

For the cross-sectional cohort designs with $S>1$, the pattern of the allocations of the optimal time points is well known in the literature (Pukelsheim, 1993). If the number of fixed regression parameters $p$ is equal to the number of cohorts $S$, then the optimal weight

for the design points is $\frac{1}{p}$. If the number of fixed regression parameters $p$ is less than the number of cohorts $S$, then $p$ of the design points have optimal weight close to $\frac{1}{p}$ while the remaining $S-p$ design points have weight close to 0 . 
Optimal number of time points $q^{*}=4$ for linear model $(p=2)$

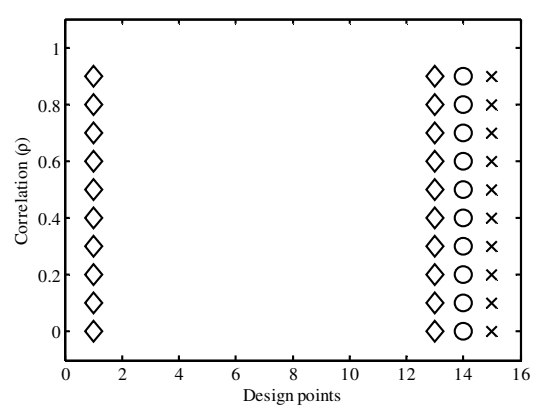

Optimal number of time points $q^{*}=5$ for quadratic model $(p=3)$

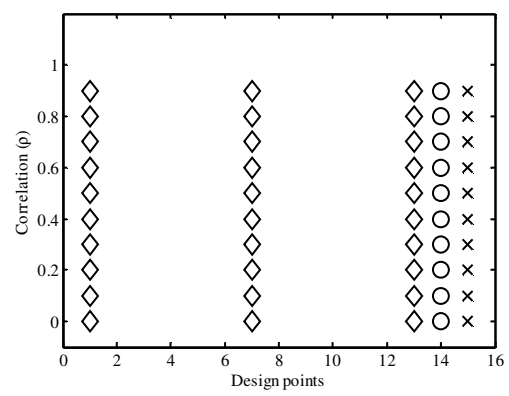

\begin{tabular}{|ll|}
\hline$\searrow$ & First cohort \\
$\bigcirc$ & Second cohort \\
$\times$ & Third cohort \\
\hline
\end{tabular}
Optimal number of time points $q^{*}=6$ for cubic model $(p=4)$

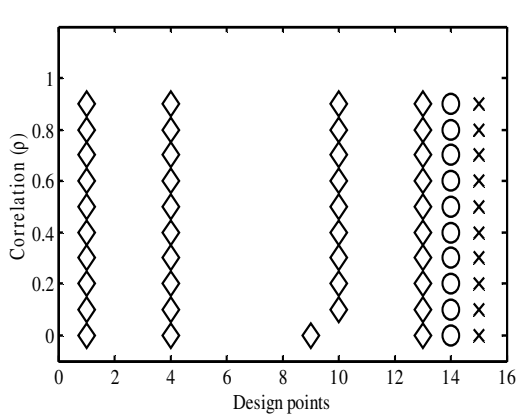

Figure 3.8. Optimal allocations for mixed longitudinal cohort design with $S=3$.

Table 3.1. Optimal weights $w_{s}$ for mixed longitudinal cohort design with $S=3$.

\begin{tabular}{|c|c|c|c|c|c|c|c|c|c|}
\hline \multirow{2}{*}{$\begin{array}{l}\text { autocor } \\
\text { relation }\end{array}$} & \multicolumn{3}{|c|}{$\begin{array}{c}\text { linear model }(p=2) \\
\text { with } q^{*}=4\end{array}$} & \multicolumn{3}{|c|}{$\begin{array}{c}\text { quadratic model }(p=3) \\
\text { with } q^{*}=5\end{array}$} & \multicolumn{3}{|c|}{$\begin{array}{c}\text { cubic model }(p=4) \text { with } \\
q^{*}=6\end{array}$} \\
\hline & $w_{1}^{*}$ & $w_{2}^{*}$ & $w_{3}^{*}$ & $w_{1}^{*}$ & $w_{2}^{*}$ & $w_{3}^{*}$ & $w_{1}^{*}$ & $w_{2}^{*}$ & $w_{3}^{*}$ \\
\hline 0.0 & 0.9139 & 0.0860 & 0.0001 & 0.9059 & 0.0940 & 0.0001 & 0.8826 & 0.1173 & 0.0001 \\
\hline 0.1 & 0.9116 & 0.0883 & 0.0001 & 0.9169 & 0.0830 & 0.0001 & 0.9030 & 0.0969 & 0.0001 \\
\hline 0.2 & 0.9112 & 0.0887 & 0.0001 & 0.9162 & 0.0837 & 0.0001 & 0.9575 & 0.0424 & 0.0001 \\
\hline 0.3 & 0.9109 & 0.0890 & 0.0001 & 0.9242 & 0.0757 & 0.0001 & 0.9675 & 0.0324 & 0.0001 \\
\hline 0.4 & 0.9131 & 0.0868 & 0.0001 & 0.9570 & 0.0429 & 0.0001 & 0.9568 & 0.0431 & 0.0001 \\
\hline 0.5 & 0.9131 & 0.0868 & 0.0001 & 0.9310 & 0.0689 & 0.0001 & 0.9666 & 0.0333 & 0.0001 \\
\hline 0.6 & 0.9197 & 0.0802 & 0.0001 & 0.9748 & 0.0251 & 0.0001 & 0.9721 & 0.0278 & 0.0001 \\
\hline 0.7 & 0.9199 & 0.0800 & 0.0001 & 0.9792 & 0.0207 & 0.0001 & 0.9761 & 0.0238 & 0.0001 \\
\hline 0.8 & 0.9227 & 0.0772 & 0.0001 & 0.9575 & 0.0424 & 0.0001 & 0.9846 & 0.0153 & 0.0001 \\
\hline 0.9 & 0.9299 & 0.0700 & 0.0001 & 0.9553 & 0.0446 & 0.0001 & 0.9944 & 0.0055 & 0.0001 \\
\hline
\end{tabular}

\section{iii) Optimal number of cohorts}

Figures 3.9 and 3.10 show the relative efficiencies of optimal cohort designs with $S>1$ compared to the purely longitudinal cohort design with $S=1$, which have an optimal number of repeated measurements for each cohort with $c_{1}=c_{2}$ and $\mathrm{c}_{1}=10 c_{2}$, respectively. In these Figures, the order of the polynomial of the model varies across the columns and the covariance matrix $\mathbf{G}$ varies across the rows, while the cohort design with its corresponding optimal number of repeated measurements varies within each plot.

From Figures 3.9 and 3.10, it can be seen that the $\mathrm{RE}<1$ and a purely longitudinal cohort design with $S=1$ is the most efficient design in all cases. This result can be 
explained by the fact that adding more correlated observations in purely longitudinal design is giving more information in describing the polynomial trend of change over time than adding more independent subjects or a cohort with independent subjects.
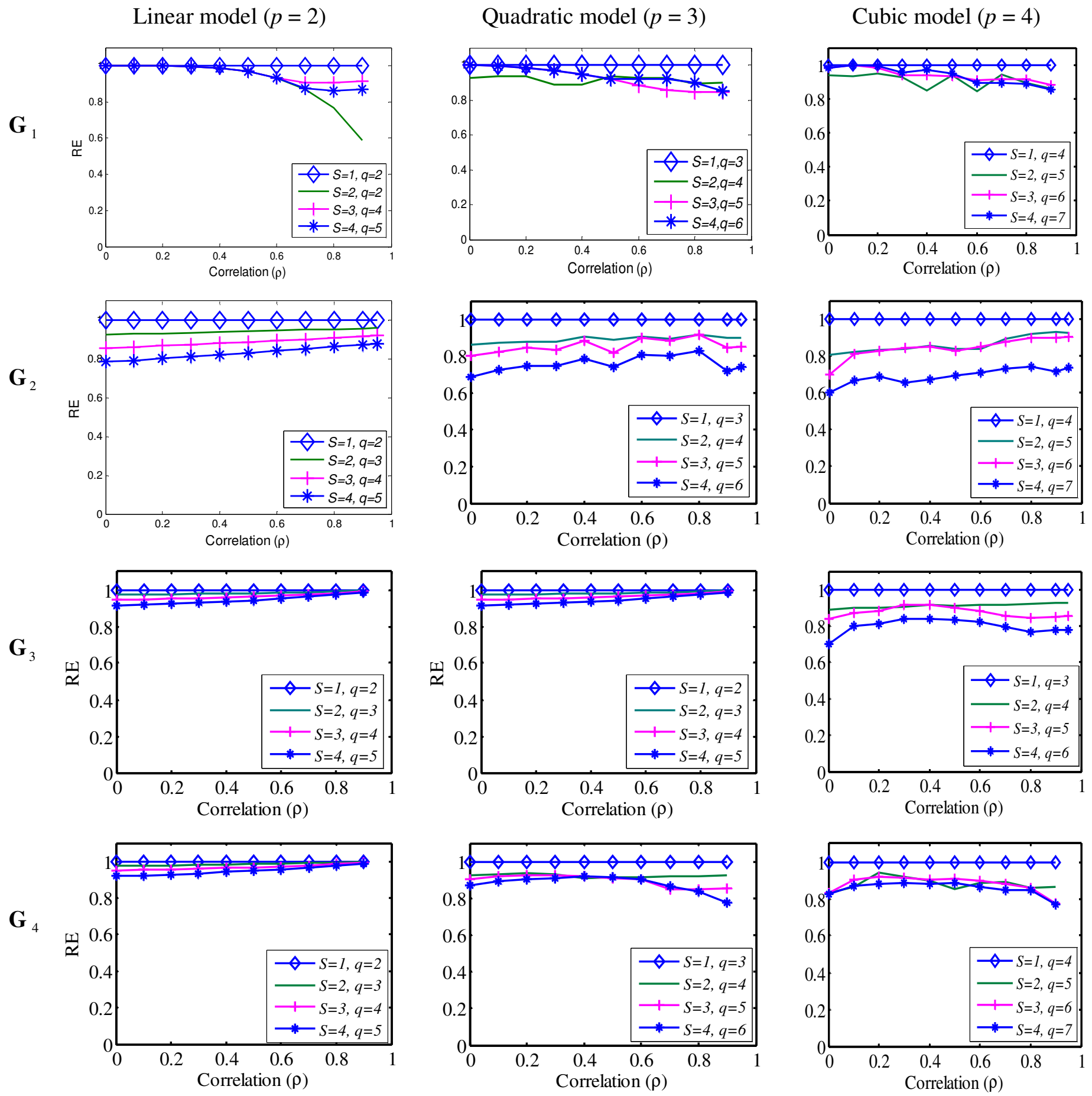

Figure 3.9. RE plots of optimal cohort designs compared to a purely longitudinal design with $c_{1}=c_{2}$. 
Linear model $(p=2)$

$\mathbf{G}_{1}$
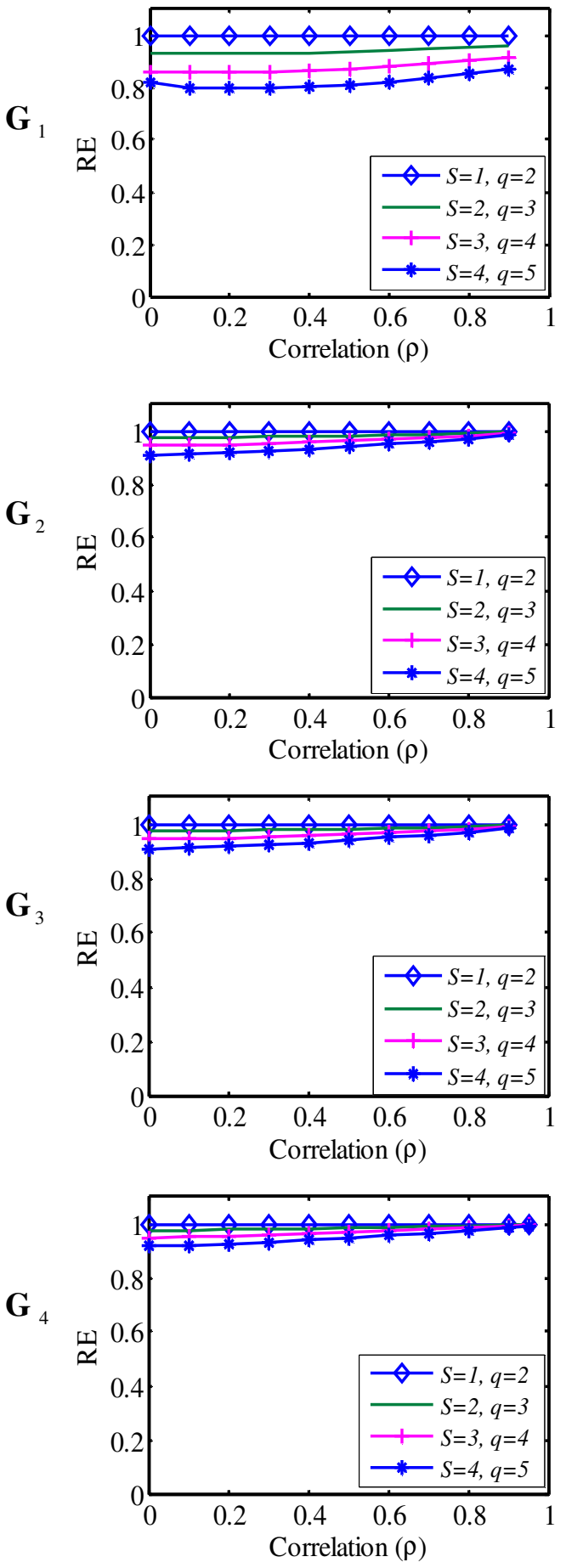

Quadratic model $(p=3)$
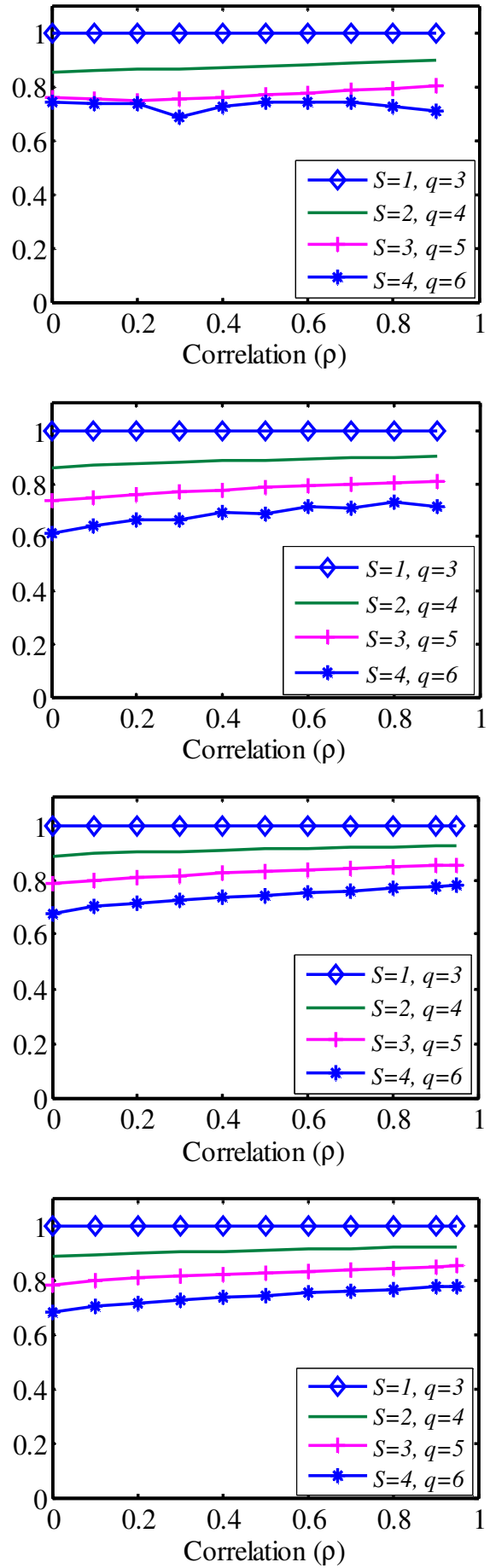

Cubic model $(p=4)$
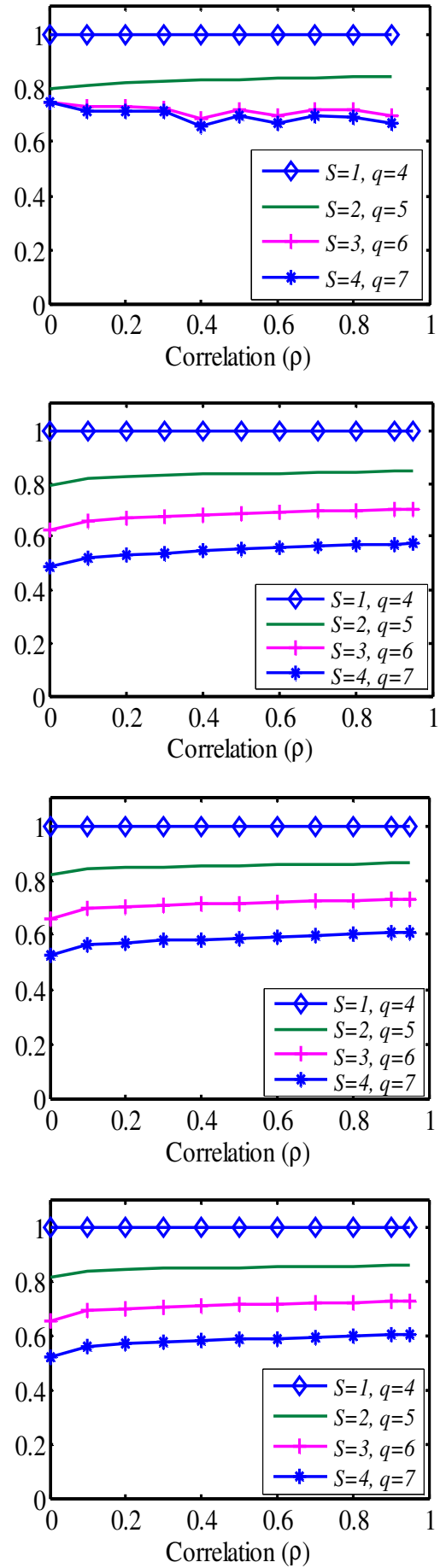

Figure 3.10. RE plots of optimal cohort designs compared to a purely longitudinal design with $c_{1}=10 c_{2}$. 


\subsection{Discussion and Conclusion}

This chapter addresses the problem of the choice of the most efficient cohort design. It emphasizes that the choice of a design should be based on the relative efficiencies of a cohort design and on the costs of the study. The results in this chapter indicate that in most cases, the most efficient number of repeated measurements is equal to $q^{*}=S+(p-1)$, i.e., the sum of the number of cohorts $S$ and the degree of the polynomial of the model ( $p$ 1). Taking $S+(p-1)$ repeated measurements at the optimal time points is not only maximizing the efficiency of the design but also saving money and easing other logistical burdens from the researchers or the subjects in the study.

The comparison of the mixed longitudinal cohort and the cross-sectional cohort designs with $S>1$ cohorts with the purely longitudinal cohort design with only $S=1$ cohort shows that the purely longitudinal cohort design is the most efficient one in all cases. The optimal allocations are similar to the findings reported by Tan and Berger (1999). The general recommendation to health science researchers is that the purely longitudinal cohort design with $p$ repeated measurements at the optimal time points is the most efficient one. If it is decided to consider more cohorts, due to more methodological considerations, then taking $S+(p-1)$ repeated measurements at the optimal time points is recommended.

It should be emphasized that the results in this chapter are restricted to models with autocorrelation, and a mixed effects structures for the random intercept, and random slope. Even though these are the commonly used models to describe longitudinal data in practice, the same methodology could be used to find optimal cohort designs for models with more complicated structures of the random effects.

The results in this chapter are also limited to the description of change over time using polynomials of at most degree 3 . These are the commonly used polynomials considered to fit most longitudinal data structures (Verbeke and Molenberghs, 2000). The general guideline in choosing $S+(p-1)$ repeated measurements at the optimal time points could also be used when higher order polynomials are considered.

This chapter focuses on the optimal designs for the estimation of parameters which describe the change over time. The results could be extended for the purpose of comparing two or more groups over time. Although we expect the results not to be very different in these cases, further study will be needed to confirm this conjecture. 


\section{Appendix: Description of the algorithm for the numerical study}

Let $\mathbf{t}_{q}^{*}=\left(t_{1}^{*}, \cdots, t_{q}^{*}\right)$ be the optimal allocation of $q$ time points,

$q^{*}$ be the optimal number of time points,

$S^{*}$ be the optimal number of cohorts,

the number of cohorts $S=1, \cdots, 4$, and

the number of time points $q \geq 2$.

The algorithm follows below for a given total cost of the study:

Step 1.

For each number of cohort $S$ and number of time points $q$,

- determine the optimal weights according to BFGS [21] local search algorithm at each combination of time points $\left\{t_{1}, \cdots t_{q}\right\}, t_{i} \in 1, \cdots, 15$, for $i=1, \cdots, q$,

- Determine the D-optimal time points $t_{q}^{*}$ based on equation (3.8) in the text.

Step 2.

Compare the RE at the D-optimal time points from step 1, and determine the most optimal number of time points $q^{*}$ for each number of cohorts $S$.

Step 3.

Compare the RE at the most optimal number of time points for each cohort from step 2, and determine the most optimal number of cohorts $S^{*}$.

Optimality check

We have used the following procedure to check optimality and we found the same optimal weights under the BFGS algorithm:

- Make a grid of weights within [0.01,0.99] at each possible combination of time points,

- compute the D-optimal solution for each possible combination of weights within the grid and determine the optimal weights. 

4 Interactive Computer Program for Optimal Designs of Longitudinal Cohort Studies

Submitted for publication 


\begin{abstract}
:
Many large scale longitudinal cohort studies have been carried out or are ongoing in different fields of science. Such studies need a careful planning to obtain the desired quality of results with the available resources. In the past, a number of researches have been performed on optimal designs for longitudinal studies. However, there was no computer-program yet available to help researchers to plan their longitudinal cohort design in an optimal way. A new user-interface computer-program for the optimization of designs for longitudinal cohort studies is therefore presented. Users can specify the model and certain parameter values for the data analysis and the program will produce the optimal design results. The computer program helps users to identify the optimal cohort design with an optimal number of repeated measurements per subject and an optimal allocations of time points within a given study period. Further, users can compute the loss in relative efficiencies of any other alternative design compared to the optimal one. The computer program is described and illustrated using a practical example.
\end{abstract}

Key words: Optimal design; D-optimal; longitudinal studies; computer program; relative efficiency; cohort designs 


\subsection{Introduction}

A user-interface computer-program for the optimization of designs for longitudinal cohort studies is presented. A longitudinal cohort study is a study that involves repeated measurements over a period of time for a group (cohort) of subjects. Longitudinal cohort studies are becoming increasingly important in many areas of psychology, health sciences and medicine among others. They are often used in psychology to study developmental trends across the life span (example, early childhood longitudinal study (http://nces.ed.gov/ecls/)). Longitudinal studies are also used in health sciences and medicine to examine changes in outcome measures over time in groups of subjects/patients and to identify associated risk factors. Examples of such studies are the Framingham heart study (Dawber, 1980), the national cancer prevention study (Calle et al., 2002), the nurses' health study (Colditz, 1994; Camargo and Willett, 1999), the New York state cohort study (Bandera et al., 1997), the longitudinal aging study of Amsterdam (Dik et al., 2000), the Survey of Medical Information and Life style study in Eindhoven- SMILE (Van den Akker et al., 2008), and the LifeLines study that follows development of health of 165,000 people in the north of the Netherlands over thirty years (http://www.lifelines.nl/).

These large scale studies all have in common that cohorts of people are measured several times over a long period of time. Researchers of such large scale studies often face the problem of how to allocate repeated measurements over time. These problems can be addressed via optimal design theory. However, these large scale studies usually have not benefited from optimal design theory. The choice of number of repeated measurements and selection of the time points at which measurements are taken in most large scale studies is often done on more or less ad-hoc basis. The costs of these large scale studies may be reduced by choosing a design more carefully and by selecting samples and cohorts more efficiently. Moreover, the number of repeated measures may also be selected to optimize the information.

The problems of optimal designs for longitudinal cohort studies have been discussed in the literature by Berger (1986), Bischoff (1993), Diehr et al. (1995), Abt et al.(1997), Mentre et al.( 1997), Atkins and Cheng (1999), Tan and Berger (1999), Ouwens et al. (2002), Berger and Tan (2004), and Tekle et al. (2007, chapter 3 of this thesis) among others. However, no user friendly software for the optimization of longitudinal studies has been developed so far. In this chapter we will introduce and describe a new interactive 
computer program for the optimal design of longitudinal studies. The basic concepts behind the program are discussed by Tekle et al. (2007). This program is self contained and will facilitate the computations.

The chapter is organized as follows. In section 4.2, design problems for longitudinal cohort studies are briefly discussed. The generalized mixed effects model and optimal designs will be described in section 4.3. Relative efficiency is defined as a measure of comparison of two designs. In section 4.4, the computer program for the optimal design of longitudinal studies will be described. A practical longitudinal study will be used to illustrate the application of the program in section 4.5. Finally, conclusion is given in section 4.6.

\subsection{Design problem for longitudinal cohort studies}

A longitudinal cohort study samples a cohort, which is defined as a group experiencing some event (birth, geographic boundary, age, sex) in a selected time period, and measures them through a time interval or study period $T$. Planners of cohort studies often face the problem of choosing between a purely longitudinal, cross-sectional or mixed longitudinal design. Depending on the number of cohorts, the cohort design may be purely longitudinal, cross-sectional or mixed longitudinal. In a purely longitudinal design one group(cohort) of subjects is measured over the study period. Alternatively, in a cross-sectional cohort design different samples (cohorts) of subjects are selected and measured at different points of time within the study period, i.e., the number of cohorts is the same as the number of time points at which the samples are taken. Sometimes, the follow up period for the purely longitudinal cohort design is considered to be too long to complete data collection. As the follow up period gets longer, the probability of dropout and non compliance may increase. If longitudinal information for subjects is still required, a mixed longitudinal design with several cohorts measured over a shorter time interval can be considered. The time intervals of these cohorts could overlap; however, for simplicity, we will assume non-overlapping time intervals.

Tekle et. al. (2007) have considered a numerical study to compare the efficiencies of these cohort designs, under certain cost considerations and found that a purely longitudinal cohort design is the most efficient one for linear mixed-effects models. As mentioned earlier, planners also face the problem of the optimal number of repeated measurements 
and their allocations in time. Tan and Berger (1999) showed that the optimal number of repeated measurements for purely longitudinal cohort design should be the same as the number of regression parameters in the model. Tekle et al. (2007) extended this result for other cohort designs and concluded that the optimal number of repeated measurement seems equal to the sum of the number of regression parameters in the model and the number of cohorts in the study minus one. The allocations of measurements within the study period mostly depend on the correlation between repeated measurements and the degree of the polynomial that describes the trend over time. If the repeated measurements are correlated and the degree of the polynomial is either linear or quadratic, then the allocation of repeated measurements is approximately equally spaced. However, if the repeated measurements are not correlated or the degree of the polynomial is cubic, then the allocation depends on the size of the correlations between the repeated measurements. If the number of repeated measurements is larger than 6 , the optimal allocations of the correlated repeated measurements are also approximately equally spaced for the cubic model.

The computer program that we will describe helps users to identify the optimal cohort design with an optimal number of repeated measurements and optimal allocations of time points. It computes the loss in efficiencies of any alternative design compared to the optimal one. The user has to specify the random effects model and the costs parameters to obtain optimal design results. In the next section, we will discuss the linear mixed-effects model to analyse the data for cohort designs. Then, the computer program will be described in section 4.4 .

\subsection{The model and optimal cohort Designs}

Suppose a researcher is interested in a longitudinal trend of a continuous response variable over time for $N$ subjects. Linear mixed effects models have been proposed by Diggle (1988), Diggle et al. (1994), Cnaan et al. (1997), and Verbeke and Molenberghs (2000), among others, for the analysis of such longitudinal data. The model is an additive model of fixed and random parts, including the error terms. The fixed part describes the group effects while the random parts describe the subject-specific effects. For a cohort design with the number of cohorts equal to $S$, the model is given by: 


$$
\mathbf{y}_{s i}=\mathbf{X}_{s} \boldsymbol{\beta}+\mathbf{Z}_{s} \mathbf{b}_{s i}+\boldsymbol{\varepsilon}_{s i},
$$

where the $q_{s} \times 1$ vector $\mathbf{y}_{s i}$ is a vector of repeated measurements on a continuous response variable for person $i, i=1, \cdots n_{s}$, at $q_{s}$ time points $\mathbf{t}_{s}=\left(t_{s 1}, \cdots, t_{s q_{s}}\right)^{\prime}$ in cohort $s, s=1, \cdots, S$ such that $\sum_{s=1}^{S} n_{s}=N$ and the total number of time points $q=\sum_{s=1}^{S} q_{s}, \mathbf{X}_{s}$ is the $q_{s} \times p$ matrix of explanatory variables including polynomials of the measurement times and $\mathbf{Z}_{s}$ is the $q_{s} \times r$ submatrix of $\mathbf{X}_{s} ; p$ and $r$ are numbers of fixed and subjectspecific regression parameters, respectively. The $p \times 1$ vector $\boldsymbol{\beta}$ is a vector of fixed regression coefficients. The $r \times 1$ vector $\mathbf{b}_{s i}$ is the vector of subject-specific coefficients with mean zero and $r \times r$ variance-covariance matrix $\mathbf{G}$. The $q_{s} \times 1$ vector $\boldsymbol{\varepsilon}_{s i}$ is the vector of random errors for subject $i$ within cohort $s$, with mean zero and variance-covariance matrix $\sigma^{2} \mathbf{R}_{s}$, where the $q_{s} \times q_{s}$ matrix $\mathbf{R}_{s}$ is the correlation matrix of the error vector and $\sigma^{2}$ is a common variance for error components. Further, it is assumed that the vectors $\mathbf{b}_{s i}$, and $\boldsymbol{\varepsilon}_{s i}$ are independent. A first order autoregressive correlation structure (AR1) is considered in the computer program for $\mathbf{R}_{s}$.

The regression parameters can be estimated using the maximum likelihood method. The interest of optimal design theory is to optimize a certain function of the variancecovariance function of the parameter estimators. The variance-covariance of the parameter estimators is given by (Cnaan et al., 1997; Tekle et al., 2007):

$$
\operatorname{var}(\hat{\boldsymbol{\beta}})=\left[\sum_{s=1}^{s} n_{s}\left(\mathbf{X}_{s}^{\prime} \mathbf{V}_{s}^{-1} \mathbf{X}_{s}\right)\right]^{-1},
$$

where $\mathbf{V}_{s}$ is the variance-covariance of the response variable in cohort $s$ and given by:

$$
\mathbf{V}_{s}=\mathbf{Z}_{s} \mathbf{G} \mathbf{Z}_{s}^{\prime}+\sigma^{2} \mathbf{R}_{s}
$$

Let the cohort designs $\xi \in \Xi_{S q}$ be defined by the form:

$$
\xi=\left\{\begin{array}{llll}
\mathbf{t}_{1} & \mathbf{t}_{2} & \cdots & \mathbf{t}_{S} \\
w_{1} & w_{2} & \cdots & w_{S}
\end{array}\right\},
$$

where $\Xi_{S q}$ is the design space of all cohort designs with $S$ cohorts such that the total number of time points within a study period is equal to $q, \mathbf{t}_{s}$ is a vector of time points $\mathbf{t}_{s}=\left(t_{s 1}, \cdots, t_{s q_{s}}\right)^{\prime}$ in cohort $s, s=1, \cdots, S$, and $w_{s}$ is the relative size of cohort $s$ such that 
$\sum_{s=1}^{S} w_{s}=1$. The interest is now to identify an optimal design among elements of $\Xi_{S q}$ that minimizes a certain function of the variance-covariance matrix of the parameter estimators. In this chapter we will restrict ourselves to optimal designs using the D-optimality criterion. The popular D-optimal design minimizes the determinant of the variancecovariance matrix of the parameter estimators.

Equation (4.2) is given for an arbitrary number of time points (repeated measurements), however, the number of measurements per subject usually needs to be restricted because of monetary cost, subject fatigue, and other logistical reasons. Therefore, it is reasonable to take the cost of measuring into account when a cohort design is optimized. Fedorov et al. (2002), Gagnon and Leonov (2005) and Tekle et al. (2007) have considered a variancecovariance matrix of the parameter estimates that is normalized for the cost parameters.

The following cost function is proposed for the total costs of a study with $S$ cohorts and $q_{s}$ repeated measurements for $n_{s}$ subjects within a cohort (Tekle et al., 2007):

$$
C=c_{0}+\sum_{s=1}^{S} n_{s}\left(c_{1}+c_{2}\left(q_{s}-1\right)\right),
$$

where $C$ is the total budget of a study, $c_{0}$ is the initial setup costs of a study and may also include the costs of maintaining the research staff during the study period, $c_{1}$ is the costs of recruiting a new subject, $c_{2}$ is the costs of a measurement at each time point for each subject.

The normalized variance-covariance matrix for a design $\xi$ which takes into account the cost parameters is:

$$
\begin{aligned}
\operatorname{var}\left(\hat{\boldsymbol{\beta}}_{\xi}\right) & =\left[\sum_{s=1}^{s} w_{s}\left(\frac{\mathbf{X}_{s}^{\prime} \mathbf{V}_{s}^{-1} \mathbf{X}_{s}}{c_{1}+c_{2}\left(q_{s}-1\right)}\right)\right]^{-1}, \\
& =\left[\sum_{s=1}^{S} w_{s}\left(\frac{\mathbf{X}_{s}^{\prime} \mathbf{V}_{s}^{-1} \mathbf{X}_{s}}{k+q_{s}-1}\right)\right]^{-1}
\end{aligned}
$$

with $w_{s}=\frac{n_{s}\left(k+q_{s}-1\right)}{C-c_{0}}, k$ is the ratio of the costs of recruiting a new subject $c_{1}$ and the costs of each measurement $c_{2}$, i.e, $k=c_{1} / c_{2}$. As can be seen in Equation (4.6), the variance-covariance matrix of the parameter estimators depends only on $k$. We will assume that the cost ratio $k=c_{1} / c_{2}>0$.

If the parameters in the variance-covariance matrix $\mathbf{V}_{s}$ and the ratio of the costs $c_{1}$ and 
$c_{2}$ are known, then the generalized variance of $\operatorname{var}\left(\hat{\boldsymbol{\beta}}_{\xi}\right)$ in equation (4.6) can be minimized over the design space $\Xi_{S q}$ by choosing a D-optimal cohort design $\xi^{*}$ such that, for each design $\xi \in \Xi_{S q}$,

$$
\operatorname{det}\left\{\operatorname{var}\left(\hat{\boldsymbol{\beta}}_{\xi^{*}}\right)\right\} \leq \operatorname{det}\left\{\operatorname{var}\left(\hat{\boldsymbol{\beta}}_{\xi}\right)\right\},
$$

where $\hat{\boldsymbol{\beta}}_{\xi^{*}}$ and $\hat{\boldsymbol{\beta}}_{\xi}$ are estimators of $\boldsymbol{\beta}$ under $\xi^{*}$ and $\xi$, respectively i.e., an optimal design is a design which for a given total cost has the smallest generalized variance of model parameter estimators.

The optimal design problem for a cohort study is to identify the optimal time allocations of the vector $\mathbf{t}_{s}$, the associated optimal weights $w_{s}{ }^{\prime} \mathrm{s}$, and the number of repeated measurements in each cohort, $q_{s}$ for cost parameters.

To compare the efficiency of two designs $\xi_{1}$ and $\xi_{2}$, the relative efficiency (RE) can be used. The RE of a design $\xi_{2}$ compared to a reference design $\xi_{1}$ is given by the ratio (Atkinson and Donev 1996):

$$
R E_{\xi_{2} \mid \xi_{1}}=\left[\frac{\operatorname{det}\left\{\operatorname{var}\left(\hat{\boldsymbol{\beta}}_{\xi_{1}}\right)\right\}}{\operatorname{det}\left\{\operatorname{var}\left(\hat{\boldsymbol{\beta}}_{\xi_{2}}\right)\right\}}\right]^{1 / p} .
$$

If the ratio is less than 1 , then $\xi_{2}$ is less efficient than $\xi_{1}$ and design $\xi_{1}$ is to be preferred compared to $\xi_{2}$. The reciprocal of the $R E_{\xi_{2} \mid \xi_{1}}$ is equal to the relative amount of extra observations that must be taken for $\xi_{2}$ to obtain the same efficiency as design $\xi_{1}$ (Atkinson and Donev 1996). For example, if $R E_{\xi_{2} \mid \xi_{1}}=0.80$, then $(1 / 0.80-1) \times 100 \%=25 \%$ more observations will be needed under design $\xi_{2}$ to have the same efficiency as under design $\xi_{1}$.

\subsection{POLS program}

\subsubsection{Introduction}

Program for Optimal design of Longitudinal Studies (POLS) is an interactive program that helps to obtain optimal cohort designs. The program runs in a MATLAB environment. The core element of the program is optimization of the generalized variance of the parameter estimates. This has been done using the fmincon function from MATLAB optimization toolbox. Fmincon is a function that finds a constrained minimum of a function of several 
variables by using a sequential quadratic programming (SQP) method (Matlab, 2004). Some of the steps of the algorithm are given in Tekle et al. (2007).

The program requires the user to choose the degree of the polynomial of a model and to provide input values on the cost parameters, the variance-covariance parameters for the random effects and error terms. Then, the user can obtain optimal allocations of time points and relative efficiencies of designs by choosing the buttons given in the main menu of the program. The output for each selected button will be displayed in a figure and/or Excel spreadsheet.

\subsubsection{Description and use of the program}

Type mainmenu in the command window of MATLAB and press the Enter key to begin with the program. Then, the menu shown in Figure 4.1 will appear. The user should give the necessary input parameters.

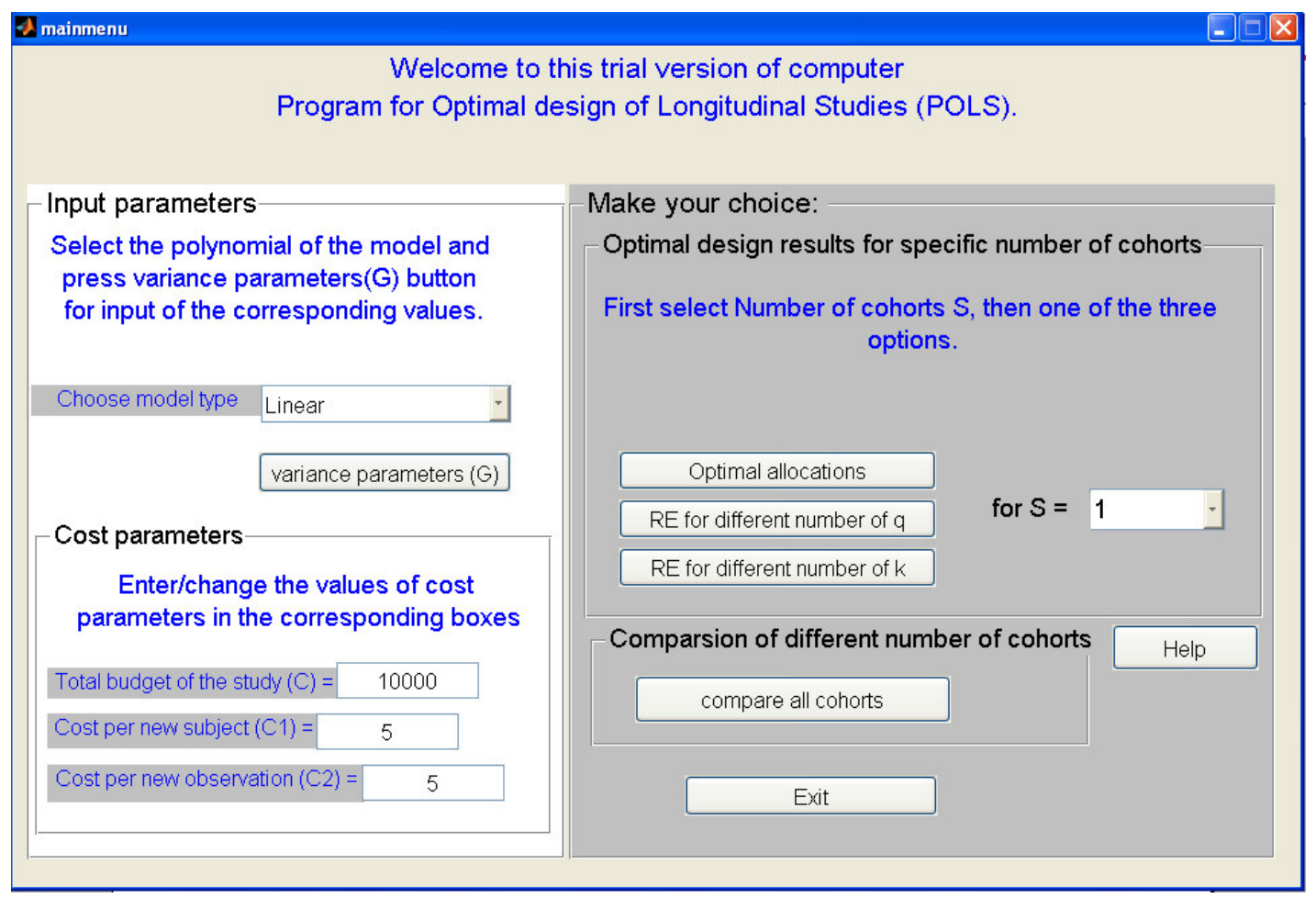

Figure 4.1. The main menu with the default input values for POLS.

The input parameters for the main menu shown in Figure 4.1 are discussed below.

\section{Input}

- Choice of a polynomial: Linear, quadratic or cubic models. This choice can be based on knowledge from previous studies, a pilot study, biological/scientific expectations or expert opinion. The default polynomial of the mixed effects model is linear. 
- Values of the variance-covariance parameters in $\boldsymbol{G}$ and the error terms: If a fixed effects model is assumed, then all the values required in $\mathbf{G}$ should be set to zero. A fixed effects model can be assumed when it is expected that the parameters that describe the trend over time do not differ between subjects. Previous numerical studies have shown that the choice of the parameter values has little or no effect on optimal designs (Tan and Berger (1999), Tekle et al. (2007)). The user will find a sub-menu, which is shown in Figure 4.2, for input of the values for the variance components in $\mathbf{G}$ matrix and the variance of the error terms.

- Values for costs parameters: the costs parameters are the total budget of the study $C$, the cost per new subject $c_{1}$, and the cost of measurement (new observation) $c_{2}$. The initial setup costs parameter $c_{0}$ is a constant in the program. As mentioned in section 4.3, the ratio $k=c_{1} / c_{2}$ plays a role in the optimization and this ratio is assumed to be greater than zero.

- The number of cohorts: the number of cohorts for a study determines the type of the cohort design. If the number of cohorts $S=1$, then the design is a purely longitudinal cohort design. On the other hand, the design will be either a mixed longitudinal or a cross-sectional cohort design when $S>1$. The design will be cross-sectional cohort design when the number of time points $q$ is equal to the number of different cohorts $S$.

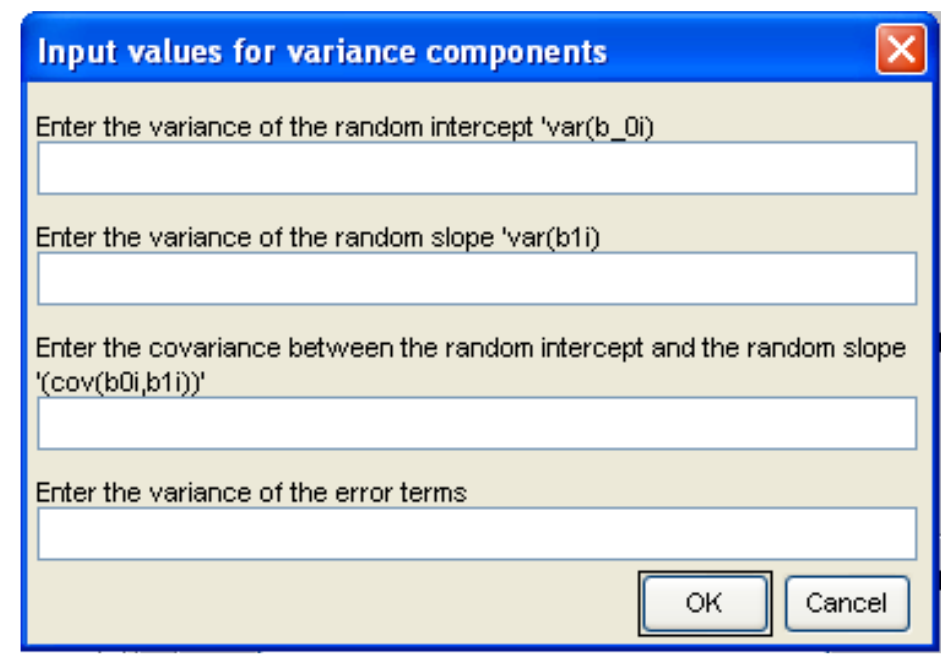

Figure 4.2. A sub-menu of POLS for input of the variance components in the G-matrix and the error terms for mixed effects models. 


\section{Buttons/options to obtain output}

- 'Optimal allocations': the user will find dialog boxes to fill the specific number of time points, and the starting and end period for the study time interval $(T)$. The optimal allocations within the selected study period for the specified number of time points will be computed.

- 'RE for different number of $q$ ': this option gives the relative efficiency of designs with the number of time points $q>p$ compared to an optimal design with the number of time points $q=p$ for the given cost parameters.

- 'RE for different number of $k$ ': the user will find an input dialog box to fill the specific number of time points $q$ at which the comparison of the values of cost ratios will be made. The relative efficiency of a design with the selected number of time points at different values of cost ratios $k$ compared to a design with number of time points $q=p$, will be computed.

- 'compare all cohorts': a sub-menu shown in Figure 4.3 will appear to provide the number of time points $q$ for each cohort design. Then, the relative efficiencies of cohort designs will be obtained.

\section{$\bullet$}

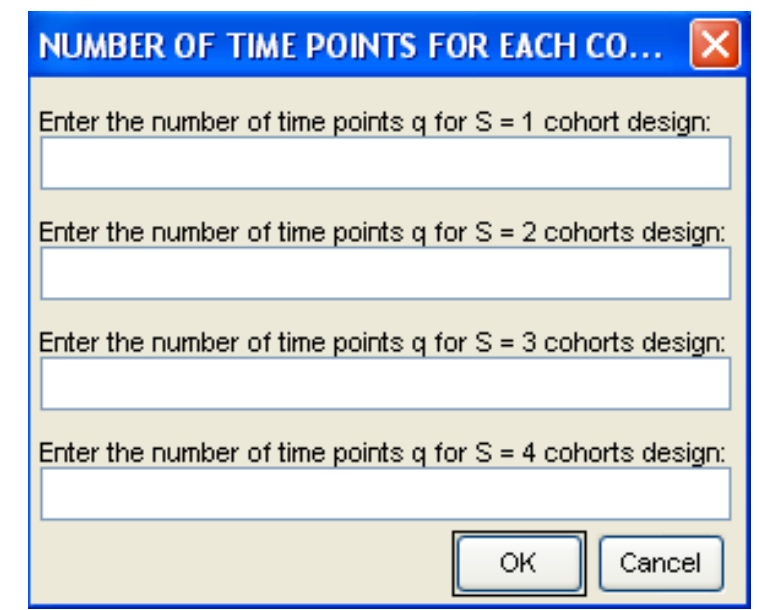

Figure 4.3. A sub-menu of POLS for input of the number of time points $q$ for cohort designs with different number of cohorts $S$.

Values with $q \geq p$ and $q \geq S$ can be filled in the boxes of the sub-menu shown in Figure 4.3. It should be noted that computer time increases exponentially as the number of cohorts and time points increases. 


\section{Main output}

The allocations of the time points and the relative efficiencies are the main results of the program. The optimal allocations and relative efficiencies are displayed in figures as a function of the autocorrelation parameter in [0, 0.95) for $\mathrm{AR}(1)$ correlation structure (Figure 4.4). These results are displayed in an editable matlab figure and if required also in an excel table. Upon request, the program opens an excel file to print the results. The output of the program contains the optimal allocations and weights $w_{s}$ of cohort designs. The output also contains the relative efficiencies comparing efficiencies of

- optimal design for $q>p$ with optimal design for $q=p$ at a specific cost ratio,

- equidistance design for $q>p$ with optimal design for $q=p$ at a specific cost ratio,

- optimal design for $q>p$ with optimal design with $q=p$ at different values of cost ratios, $k$,

- cohort designs for $S>1$ with a purely longitudinal cohort design $(S=1)$.

Once the POLS computer program has started, a user can change input values or obtain results by pressing the corresponding buttons on the main menu (Figure 4.1) as many times as one wishes. A 'Help' button is also available for guidance. The 'Exit' button in the main menu stops the program.

\subsection{Illustration: Design on height of schoolgirls}

Verbeke and Molenberghs (2000) have used mixed effects model to analyse the growth curves of 20 preadolescent schoolgirls previously reported and analysed by Goldstein (1979) with a fixed effect model. The heights of the girls are measured on a yearly basis from age 6 to 10. The design is a purely longitudinal design with 5 repeated measurements within the time interval $[6,10]$. Verbeke and Molenburghs (2000) have assumed that the growth of girls is a linear function of age (time) and they have found that a random intercept and random slope model gives the best fit to the data. We adopt this model and use the POLS program to compute the relative efficiency of this design.

The values reported from the analysis of the data by Verbeke and Molenburghs (2000) are used for the parameters in G, i.e., $\operatorname{var}\left(b_{0}\right)=7.603, \operatorname{var}\left(b_{1}\right)=0.133$, $\operatorname{cov}\left(b_{0}, b_{1}\right)=-0.444$ and $\sigma^{2}=0.476$. For this illustration we will assume that these 
values are available from previous data and they are reliable estimates for the design of a future study. Note that the choice of these values will not have a noticeable difference in the optimal allocations of time points (Tan and Berger, 1999; Tekle et al., 2007). The default values given in Figure 4.1 for the cost parameters with $S=1$ cohort are used.

Since there are $p=2$ regression parameters in the mixed effects model with linear trend, the optimal number of time points is also $q=2$. The allocation of $q=p=2$ optimal design points is at the end points of the time interval (Figure 4.4a).

The relative efficiency of a design with more repeated measurements $q>p$ can be obtained by choosing the 'RE for different number of $q$ ' button. Figure $4.4 \mathrm{~b}$ shows the RE for all designs with number of time points $q \geq p$.

a) optimal allocation of design points

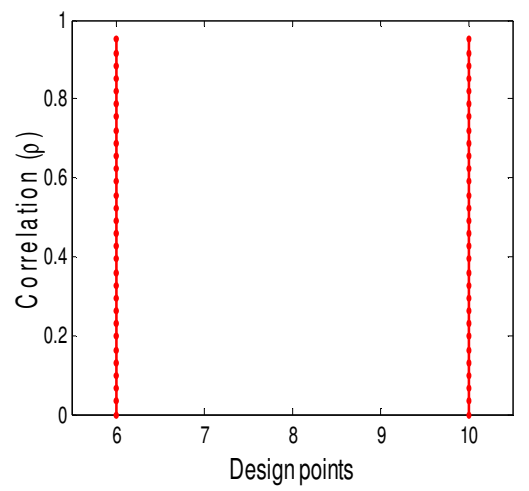

b) RE of designs with $q$ different time points

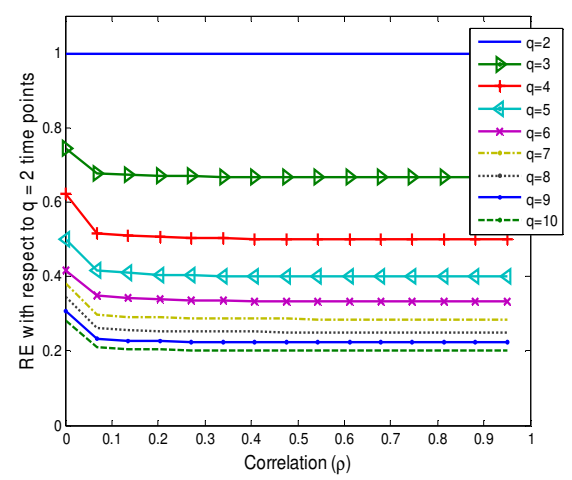

c) RE of the design with $q=5$ time points compared to the optimal design with $q=2$ time points for different $k$

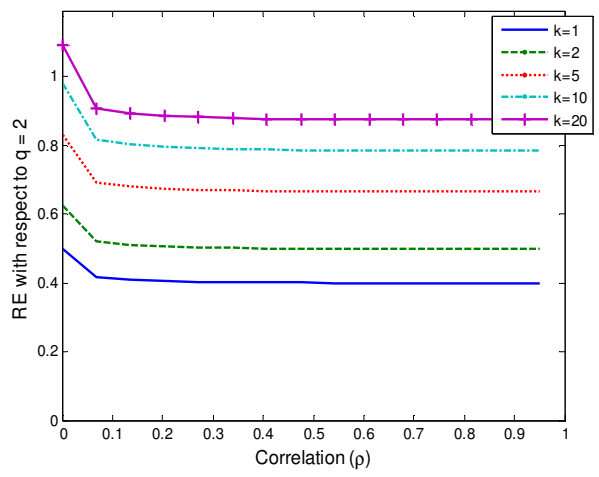

Figure 4.4. Results of the school girls example based on linear mixed effects model with $p=2$.

It is also possible to obtain the relative efficiency of a design with 5 measurements compared to the optimal number of repeated measurements for different costs ratios $k$. Figure 4.4c, the RE at different values of the costs ratio for a design with five repeated measurements compared to a design with 2 repeated measurements. As it can be seen from the plot in Figure 4.4c that the RE increases as the costs ratio increases, because obtaining an additional observation becomes relatively cheaper as $k$ increases. However, the RE remains below one for correlated measurements. When the correlation parameter is zero (uncorrelated measurements), the RE could become greater than 1 for costs ratio $k>20$. Tekle et al. (2008) have shown that when the costs ratio $k=c_{1} / c_{2} \rightarrow \infty$, the RE can be greater than 1 for $\rho=0$; however, it remains below 1 if $\rho>0$.

Tekle et al. (2007) have shown that the optimal number of time points $q$ is mostly equal 
to $S+(p-1)$ for a cohort design with $S$ cohorts, where $p$ is the number of regression parameters in the model. Suppose that cohort designs with more cohorts are planned for the growth of girls' study. The efficiency of the cohort designs can be compared by using values $q=2,3,4$, and 5 for cohorts with number of cohorts $S=1,2,3$, and 4, respectively, in the submenu shown in Figure 4.3. Note that these values of $q$ are the optimal number of time points at the corresponding number of cohorts $S$ for linear mixed effects model with number of regression parameters $p=2$. Figure 4.5 shows the relative efficiencies of cohort designs with different number of cohorts $S$. From this figure, the relative efficiencies are less than 1 for cohorts designs with $S>1$ cohorts implying that the purely longitudinal design with $S=1$ is the most efficient design.

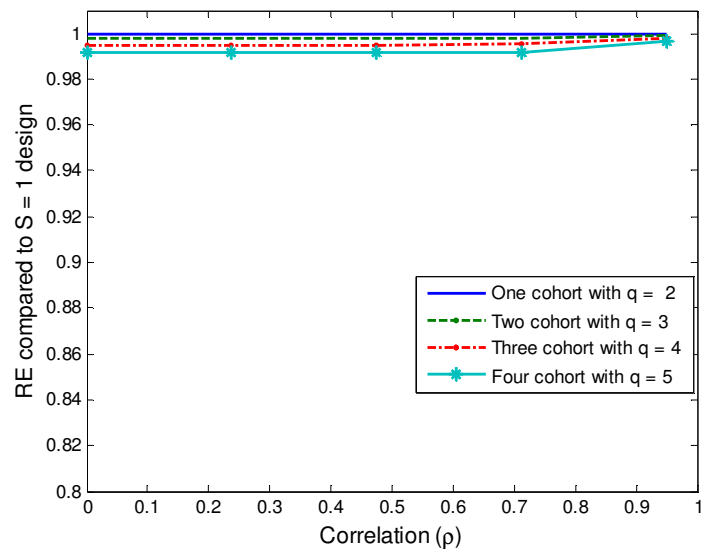

Figure 4.5. REs of optimal cohort designs compared to $S=1$.

\subsection{Conclusion}

Longitudinal cohort studies are usually expensive and they require a careful planning. Planners should use optimal design theory to select the optimal number of repeated measurements and the optimal spacing of the measurements over time. A user-friendly computer program to obtain optimal design results is described in this chapter. The user can specify the model and the cost parameters and obtain results on optimal cohort designs for a mixed-effects model. The computer program can also provide the relative efficiencies of alternative designs which are not optimal.

The current version of the computer program can be obtained from the authors upon request. One of our future works is to make the program available via internet. The current version of the program considers designs based on the D-optimality criterion. Our future work is to include other optimality criteria as well. 
5 Highly Efficient Designs for Logistic Models with Categorical Variables

Tekle, F.B., Tan, F.E.S., and Berger, M.P.F. (2008). Communications in Statistics- Theory and Methods, 37 (5): 746 - 759. 


\begin{abstract}
:
Optimal designs for logistic models generally require prior information about the values of the regression parameters. However, experimenters usually do not have full knowledge of these parameters. We propose a design which is D-optimal on a restricted design region. This design assigns an equal weight to design points which contain more information and ignores those design points which contain less information about the regression parameters. The design can be constructed in practice by means of the rank order of the outcome variances. A numerical study compares the proposed design with the D-optimal and completely balanced designs in terms of efficiency.
\end{abstract}

Keywords: optimal design; logistic model; D-optimal; restricted design space; rank based designs; relative efficiency 


\subsection{Introduction}

In optimal design theory, a design which maximizes parameter information by minimizing the variance of the parameter estimates is chosen as an optimal design. Several optimality criteria have been proposed for the design of experiments for linear models. However, these optimality criteria depend on the unknown parameter values in the case of non-linear models like logistic regression (Fedorov, 1972; Pukelsheim, 1983; Silvey, 1980; Atkinson and Donev, 1996). This parameter dependency of the optimality criteria makes the design of experiments for logistic models more complicated. Three approaches have been suggested to overcome the parameter dependency; a sequential procedure that uses information from previous runs (Sitter and Forbes, 1997; Zhu et al., 1998), a Bayesian procedure that assumes a prior distribution for the parameters (Dette, 1996), and a minimax approach which minimizes the worst possible case over a user selected region of parameters (King and Wong, 2000; Ouwens et al., 2002).

In this chapter, we will present highly efficient designs using an alternative approach especially useful for the logistic models with categorical variables. Our method first imposes a restriction on the design region, and then finds a design that maximizes the optimality criterion in the restricted region. Only information about the rank orders of the outcome variances at the design points are used to construct the designs. Thus, no precise knowledge of the model parameter values is needed in advance. In those cases where a sequential approach may not be feasible in practice and it may not be possible to adequately specify a prior distribution of the parameters in a Bayesian approach, this procedure may be applied.

The chapter is organized as follows. First, we will discuss the model and the optimality criterion in section 5.2. We will describe the D-optimal design for logistic regression models with categorical independent variables and give a lemma on the D-optimal design for saturated models. The proposed designs are described in section 5.3. A property of the proposed designs and the corresponding algorithm to construct these designs in practice are given in the same section. A numerical study to compare the relative efficiencies of the completely balanced and the proposed designs compared to the D-optimal designs is given in section 5.4. Finally a general discussion is given in section 5.5. 


\subsection{The Model and the D-Optimality Criterion}

Assume that there are $s$ independent categorical variables and $h$ design points. Subjects are evaluated for the binary outcome variable with values 0 and 1 at the $h$ design points. Then, the logit link function relates the mean outcome to the linear predictor

$$
\eta_{i}=\log \left(\frac{\pi_{i}}{1-\pi_{i}}\right)=\sum_{j} x_{i j} \beta_{j}, \quad i=1, \cdots, N,
$$

where $\pi_{i}$ is the probability that the outcome is 1 for the $i^{\text {th }}$ subject. In total there are $N$ subjects in the study. Details about the logit link function $\eta_{i}$ are given by McCullagh \& Nelder (1989). It is convenient to express the model as follows:

$$
\boldsymbol{\eta}=\mathbf{X} \boldsymbol{\beta}
$$

where $\boldsymbol{\eta}$ is an $N \times 1$ vector, with the logit link function $\eta_{i}$ as the $i^{\text {th }}$ element. The $N \times p$ design matrix $\mathbf{X}$ has known values 0 and 1 for the intercept, the dummy variables and interactions among the variables, where $p$ is the number of parameters in the model, $p \leq h \leq N$. Row $i$ of $\mathbf{X}$ represents the design point $\mathbf{x}_{i}$ with coordinates $\mathbf{x}_{i}=\left(1, x_{i 1}, \cdots, x_{i(p-1)}\right)^{\prime}$ for each subject. The $p \times 1$ vector $\boldsymbol{\beta}=\left(\beta_{0}, \beta_{1}, \cdots, \beta_{p-1}\right)^{\prime}$ contains the regression parameters.

The asymptotic variance-covariance of the maximum likelihood estimator $\hat{\boldsymbol{\beta}}$ is given by (McCullagh and Nelder, 1989):

$$
\operatorname{var}(\hat{\boldsymbol{\beta}})=\left(\mathbf{X}^{\prime} \mathbf{V X}\right)^{-1},
$$

where $\mathbf{V}=\operatorname{diag}\left\{\pi_{i}\left(1-\pi_{i}\right)\right\}, i=1, \cdots, N$.

An approximate design $\xi$, which is an element of the design space $\Xi$, with $h$ distinct design points for model (5.2) is given by:

$$
\xi=\left\{\begin{array}{llll}
\mathbf{x}_{1} & \mathbf{x}_{2} & \cdots & \mathbf{x}_{h} \\
w_{1} & w_{2} & \cdots & w_{h}
\end{array}\right\}
$$

where $\mathbf{x}_{1}, \mathbf{x}_{2}, \cdots, \mathbf{x}_{h}$ are the design points, and $w_{1}, w_{2}, \cdots, w_{h}$ are the associated weights or proportions of subjects at the $h$ design points, respectively. Since for categorical variables the design points are fixed, the design problem is now to optimally choose the weight $w_{k}$, for $k=1, \cdots, h$, under the constraint that $\sum_{k=1}^{h} w_{k}=1$. The asymptotic variance-covariance of $\hat{\boldsymbol{\beta}}$ which is given in (5.3) can be normalized for the total sample size $N$ and can be expressed as follows: 


$$
\operatorname{var}\left(\hat{\boldsymbol{\beta}}_{\xi}\right)=N \operatorname{var}(\hat{\boldsymbol{\beta}})=\left(\sum_{k=1}^{h} w_{k} v_{k} \mathbf{x}_{k} \mathbf{x}_{k}^{\prime}\right)^{-1},
$$

where $v_{k}$ is the variance of the outcome variable at the $k^{\text {th }}$ design point, which is given by $v_{k}=\pi_{\mathbf{x}_{k}}\left(1-\pi_{\mathbf{x}_{k}}\right)$, where $\pi_{\mathbf{x}_{k}}$ is the probability that the outcome is 1 for the $k^{\text {th }}$ design point. The amount of information in a design is measured by the Fisher information matrix, which is equal to the inverse of the variance-covariance matrix of the parameter estimates.

A design $\xi^{*} \in \Xi$ is said to be D-optimal if (see Atkinson and Donev (1996) among others)

$$
\min _{\xi \in \Xi} \operatorname{Det}\left\{\operatorname{var}\left(\hat{\boldsymbol{\beta}}_{\xi}\right)\right\}=\operatorname{Det}\left\{\operatorname{var}\left(\hat{\boldsymbol{\beta}}_{\xi^{*}}\right)\right\},
$$

where $\operatorname{Det}\left\{\operatorname{var}\left(\hat{\boldsymbol{\beta}}_{\xi}\right)\right\}$ is the determinant of the asymptotic variance-covariance of $\hat{\boldsymbol{\beta}}$ under the design $\xi$.

It should be emphasized that the variances of binary outcomes $v_{k}$ are functions of the regression parameters $\boldsymbol{\beta}$ (Equation 5.2). This means that the variance-covariance matrix in (5.5) and the optimality criterion in (5.6) are also functions of $\boldsymbol{\beta}$. To find and implement the D-optimal design, one therefore must know the values of these parameters. The Doptimal designs that could be obtained at specific values of the parameters are only locally optimal for the specific parameter values (see Atkinson and Donev (1996) among others). An exception to this is when the model is saturated, i.e. when the number of design points is equal to the number of regression parameters. No prior knowledge about the regression parameter values is then needed (see lemma 5.1.3 of Silvey (1980) for linear models and the lemma given below for the logistic models with categorical independent variables). In the next section we will describe D-optimal designs for a restricted design space.

\section{Lemma}

If $\mathbf{Z}$ is a $p \times p$ nonsingular matrix with rank $p$ in the logistic model

$$
\boldsymbol{\eta}=\mathbf{Z} \boldsymbol{\beta}
$$

with $p$ regression parameters, then the D-optimal design is balanced. In the next section we will describe D-optimal designs for a restricted design space.

\subsection{Restricted D-Optimal Design for Logistic Model}

The motivation for the restricted D-optimal design arises from the fact that the variance- 
covariance matrix in equation (5.5) of the parameter estimates for the logistic model is inversely proportional to the variances $v_{k}$ of the outcome of the subjects. Thus, if we have an idea about the variances of the outcome measurements, the determinant of the variancecovariance matrix of the parameter estimates can be minimized by allocating all subjects to a combination of design points to estimate the parameters efficiently in such a way that the product of the variances of the outcomes are expected to be large and no subjects to the remaining design points.

Suppose that we have $h$ distinct design points $\left(\mathbf{x}_{1}, \cdots, \mathbf{x}_{h}\right)$ with $p$ unknown regression parameters in model (5.2). A design which assigns an equal weight to only $p$ design points and no weight to the remaining $(h-p)$ design points, will avoid the problem of parameter dependency. Following the lemma in the previous section, this design will be D-optimal for the restricted design space with $(h=p)$ design points. The problem is now to optimally select $p$ design points out of the available $h$ design points. To enable this we will modify the optimality criterion given in (5.6) by imposing a restriction on the design space. This optimality criterion is used to define a so called restricted D-optimal design.

A design $\xi_{r}^{*} \in \Xi_{R}$ is a restricted D-optimal design if

$$
\min _{\xi_{r} \in \Xi_{R}} \operatorname{Det}\left\{\operatorname{var}\left(\hat{\boldsymbol{\beta}}_{\xi_{r}}\right)\right\}=\operatorname{Det}\left\{\operatorname{var}\left(\hat{\boldsymbol{\beta}}_{\xi_{r}^{*}}\right)\right\}
$$

where $\Xi_{R} \subset \Xi$ is the set of all possible experimental designs with the constraint that nonzero weights are assigned to $p$ independent design points and the remaining $(h-p)$ design points receive zero weights, $\operatorname{var}\left(\hat{\boldsymbol{\beta}}_{\xi_{r}}\right)$ and $\operatorname{var}\left(\hat{\boldsymbol{\beta}}_{\xi_{r}^{*}}\right)$ are the asymptotic variance-covariance of $\hat{\boldsymbol{\beta}}$ under the designs $\xi_{r}$ and $\xi_{r}^{*}$, respectively. Note that the restricted D-optimal design uses the D-optimality criterion. A restricted D-optimal design is D-optimal for a restricted design space, but is sub-optimal for the unrestricted design space.

The following property with proof outlined in the appendix, helps to identify the restricted D-optimal design that satisfies equation (5.8).

\section{Property}

Suppose that $p<h$ and that $\xi_{r}^{*} \in \Xi_{R}$ is a design that assigns equal weights to $p$ independent design points and zero weights to the remaining $(h-p)$ design points, such that $\left(\operatorname{Det}\left\{\mathbf{Z}_{l_{\max }}\right\}\right)^{2} \prod_{k=1}^{p} v_{k}$ is largest among all possible $p$ independent design points out of 
the total $h$ design points, where $\mathbf{Z}_{l_{\max }}$ is the $p \times p$ design matrix for the design $\xi_{r}^{*}$; and $v_{k}$ is the variance of the outcome at the design point $\mathbf{x}_{k}$ with coordinates given in the $k^{\text {th }}$ row of $\mathbf{Z}_{l_{\max }}$. Then, $\xi_{r}^{*}$ is the restricted D-optimal design.

\section{Approximation to restricted optimal design}

The values of the variances $v_{k}$ are usually not exactly known in practice. In many cases a researcher may be able to supply information that could be translated in terms of the rank order of the outcome variances. Then rank orders of the $v_{k}$ 's can be used instead of their actual values to obtain an approximation of the restricted D-optimal design. We will refer to the obtained design as an approximation of the restricted D-optimal design and it is denoted by r-design, because the objective function is based on the rank order of the variances. An algorithm is given in section 5.3.2 for the construction of the r-designs in practice. The following can be noted about the approximate r-design;

- one needs only the rank order of the variances to construct approximate r-designs,

- the efficiency of the restricted D-optimal design is always larger than or equal to that of a design that maximizes the criterion $\left(\operatorname{Det}\left\{\mathbf{Z}_{l_{\max }}\right\}\right)^{2} \prod_{k=1}^{p} \operatorname{rank}\left(v_{k}\right)$, where $\operatorname{rank}\left(v_{k}\right)$ is the rank order of the outcome variance $v_{k}$.

It will be shown in our numerical analysis that such a design is highly efficient in practice. An r-design can be obtained by ranking variances. We will illustrate below, that researchers in applied area usually have some prior information about the direction of the effects in the study which will make it possible to rank the variances at the design points.

\subsubsection{Ranking the Outcome Variances}

To see how information about rank order of outcome variances can be extracted in practice, consider a design of a clinical trial for the treatment of primary breast cancer after a radical mastectomy. In addition to the treatment factor, a major prognostic factor to be controlled is the nodal status (i.e. number of positive axillary nodes). This example is comparable to the one given by Pocock (1983, p 82). By considering patients' disease free status $(1=$ yes, $0=$ no) after two years of the treatment as an end point for the trial, the following logistic model is specified: 


$$
\operatorname{logit}\left(\pi_{i}\right)=\beta_{0}+\beta_{1} \text { treatment }+\beta_{3} \text { nodal status . }
$$

The parameter $\pi_{i}$ is the disease free probability of patient $i=1, \cdots, N$ after two years, treatment has two categories (new drug and placebo), and nodal status has two groups (1-3 and $\geq 4$ ). For model (5.9), there are $h=4$ design points that correspond to the four different combinations of treatment and nodal status levels, i.e. the design points are:

$\mathbf{x}_{1}$ : new drug, nodal status $1-3, \mathbf{x}_{2}$ : new drug, nodal status $\geq 4$,

$\mathbf{x}_{3}$ : placebo, nodal status $1-3$, and $\mathbf{x}_{4}$ : placebo, nodal status $\geq 4$.

The rank ordering of the outcome variances $v_{k}$ 's can be determined by means of the following prior expectations. Clinicians often have prior expectations. In this example the following can be hypothesized:

- the probability that patients are disease free after two years is greater than 0.5;

- for both treatment groups, the probability that patients with nodal status 1-3 are disease free is at least equal to that of the patients with nodal status $\geq 4$;

- the probability that patients with nodal status $\geq 4$ receiving the new drug are disease free after two years is greater than that of patients with nodal status 1-3 not receiving the new drug.

Based on these prior expectations, it can be inferred that $\pi_{\mathbf{x}_{1}}>\pi_{\mathbf{x}_{2}}>\pi_{\mathbf{x}_{3}}>\pi_{\mathbf{x}_{4}}$, where $\pi_{\mathbf{x}_{k}}$ is the disease free probability at the design point $\mathbf{x}_{k}, k=1, \cdots, 4$. Because $v_{k}=\pi_{\mathbf{x}_{k}}\left(1-\pi_{\mathbf{x}_{k}}\right)$, it follows that $v_{k}$ 's can be ordered as $v_{1}<v_{2}<v_{3}<v_{4}$.

\subsubsection{Algorithm}

The following algorithm helps to construct designs that lead to the lower bound efficiency of restricted D-optimal designs.

1. Rank the (approximate) outcome variances of the $h$ design points and assign a rank $\operatorname{order} k=\operatorname{rank}\left(v_{k}\right)$ to each design point, i.e. $v_{1} \leq \cdots \leq v_{h}$, with rank order $k=1, \cdots, h$.

2. Rank the collection $\mathbf{Z}_{l}$ of all possible selections of $p$ design points out of $h$ design points according to the product of the square of the determinant and the ranks of the variances, $\left(\operatorname{Det}\left\{\mathbf{Z}_{l}\right\}\right)^{2} \prod_{k=1}^{p} \operatorname{rank}\left(v_{k}\right)$. Note that the $v_{k}$ 's are the variances corresponding to the rows of $\mathbf{Z}_{l}$. The collection is the $p \times p$ matrix $\mathbf{Z}_{l}$ and it must be of full rank. 
3. Select $\mathbf{Z}_{l_{\max }}$ with $\max \left(\operatorname{Det}\left\{\mathbf{Z}_{l}\right\}\right)^{2} \prod_{k=1}^{p} \operatorname{rank}\left(v_{k}\right)$.

4. Assign equal weights to the design points in $\mathbf{Z}_{l_{\max }}$ and assign zero weights to the remaining $(h-p)$ design points.

The design obtained from this algorithm is a r-design as an approximation of the restricted D-optimal design. By applying this algorithm for the clinical trial example discussed in section 5.3.1, the r-design will assign zero weight to design point $\mathbf{x}_{1}$ and an equal weight to the remaining three design points.

Designs can be compared using the relative efficiencies. The relative efficiency of an arbitrary design $\xi$ relative to the D-optimal design $\xi^{*}$ is defined as (see Atkinson and Donev (1996) among others):

$$
\operatorname{RE}_{\xi \mid \xi^{*}}=\left(\frac{\operatorname{Det}\left\{\operatorname{var}\left(\hat{\boldsymbol{\beta}}_{\xi^{*}}\right)\right\}}{\operatorname{Det}\left\{\operatorname{var}\left(\hat{\boldsymbol{\beta}}_{\xi}\right)\right\}}\right)^{1 / p} .
$$

This efficiency measure is proportional to the sample size and can be applied to determine the required sample size for a design $\xi$ to have the same efficiency as the optimal design $\xi^{*}$. For instance; twice as many observations are needed for a design with $\mathrm{RE}_{\xi \xi \xi^{*}}=0.50$ to be as efficient as the D-optimal design.

\subsection{Numerical Study and Results}

The numerical study will compare the D-optimal, the r-design and completely balanced designs for logistic models. The locally D-optimal designs are constructed for specific parameter values. The corresponding r-designs are constructed using the described algorithm. The completely balanced designs are constructed by assigning equal weights to the $h$ design points. The relative efficiencies of the r-designs and completely balanced designs with respect to the D-optimal design are computed.

\subsubsection{Models for the Numerical Study}

Table 5.1 shows the number of different non-saturated $(p<h)$ logistic models that can be specified with at most three categorical independent variables each having at most three levels. We will consider a total of 23 models in this numerical study. 
Table 5.1. Non-saturated logistic models with different combinations of levels of the independent variables grouped in 7 classes.

\begin{tabular}{|c|c|c|c|c|c|c|}
\hline \multirow{2}{*}{$\begin{array}{l}\text { Class of } \\
\text { models }\end{array}$} & \multicolumn{3}{|c|}{ Number of levels } & \multirow{2}{*}{$\begin{array}{l}\text { Number of only } \\
\text { main effects models }\end{array}$} & \multirow{2}{*}{$\begin{array}{l}\text { Number of models with } \\
\text { two-way interactions }\end{array}$} & \multirow{2}{*}{$\begin{array}{l}\text { Total number } \\
\text { of models }\end{array}$} \\
\hline & $X_{1}$ & $X_{2}$ & $X_{3}$ & & & \\
\hline 1 & 2 & 2 & 0 & 1 & - & 1 \\
\hline 2 & 2 & 3 & 0 & 1 & - & 1 \\
\hline 3 & 2 & 2 & 2 & 1 & 3 & 4 \\
\hline 4 & 2 & 2 & 3 & 1 & 5 & 6 \\
\hline 5 & 3 & 3 & 0 & 1 & - & 1 \\
\hline 6 & 3 & 3 & 2 & 1 & 5 & 6 \\
\hline 7 & 3 & 3 & 3 & 1 & 3 & 4 \\
\hline \multicolumn{4}{|c|}{ Total } & 7 & 16 & 23 \\
\hline
\end{tabular}

There are 7 models with only main effects, one from each class of models, and 16 different types of two-way interaction models. For example, in the sixth class of models, there are two variables $X_{1}$ and $X_{2}$ each having three levels and a third variable $X_{3}$ with two levels. For this class of models, there is one logistic model that contains only main effects and there are five different interaction models that contain the following two-way interactions in addition to the main effects:

i) $X_{1} X_{2}$,

ii) $X_{1} X_{3}$ or $X_{2} X_{3}$,

iii) $X_{1} X_{3}+X_{2} X_{3}$,

iv) $X_{1} X_{2}+X_{1} X_{3}$ or $X_{1} X_{2}+X_{2} X_{3}$,

v) $X_{1} X_{2}+X_{1} X_{3}+X_{2} X_{3}$.

Note that models with the same structure (as the two models in each cases of ii and iv above) are counted as a single model in Table 5.1. Dummy variables for each categorical variable $X$ should be used for the specification of the logistic model. For example, the model in the sixth class that contains the interactions $X_{1} X_{3}$ and $X_{2} X_{3}$ is specified as

$$
\begin{aligned}
\operatorname{logit}(\pi)= & \beta_{0}+\beta_{1} D_{11}+\beta_{2} D_{12}+\beta_{3} D_{21}+\beta_{4} D_{22}+\beta_{5} D_{31} \\
& +\beta_{6} D_{11} * D_{31}+\beta_{7} D_{12} * D_{31}+\beta_{8} D_{21} * D_{31}+\beta_{9} D_{22} * D_{31},
\end{aligned}
$$

where $D_{j m}$ is the $m^{\text {th }}$ dummy variable for the categorical variable $X_{j}$. Note that, $X_{1}$ and $X_{2}$ have two dummy variables and $X_{3}$ has only one.

\subsubsection{Model Parameters for the Numerical Study}

To construct locally D-optimal designs for the models in Table 5.1 we need prior 
knowledge about the values of the regression parameters. Parameter values are related to odds ratios and theoretically they can be any real number. However, in many studies odds ratios were found to be less than twenty (see Astin (1981), Carr et al. (2000), Deeks et al. (1999), Moroldo et al. (2004) among others). Therefore, we will only consider odds ratios less than 20 , i.e. parameter values less than $\ln (20)$. Only positive parameter values need to be specified, because D-optimal designs for logistic models are invariant to scale transformation of the variables. See Ouwens et al. (2006) for a proof.

We have considered all possible combinations of the parameter values within the range $[0.10,3.00]$. When models with interactions are involved, odds ratio values greater than 20 may occur and such cases are excluded.

\subsubsection{Results of the numerical study}

Results of the numerical study have a similar pattern for all models in Table 5.1. Therefore, we will only report results of one model from each class of models. The results are also similar for different values of the intercept parameter and we will report the relative efficiency plots only for $\beta_{0}=0.10$ and 3.00. These values are translated in to outcome probabilities 0.52 and 0.95 , respectively at the reference design point where all dummy variables are zero. Results for main effects models and models with interaction terms will be described separately.

\section{Results for Only Main Effects Models}

The relative efficiency plots for only main effects models are given in Figures 1-5.

In these figures, specific values of odds ratios vary across the columns and the values of the intercept parameter varies across the rows, while the relative efficiencies for the completely balanced (solid lines) and r-designs (dashed lines) are shown at each plot in the cells of the figures. In each plot the relative efficiencies (RE) are given at the vertical axis and the horizontal axis shows the values of the odds ratios for one of the factors in the model. The horizontal axis can also be seen as the values of regression parameter for one of the factors in the model. These regression values range from 0.10 to 3.00. 
the other odds ratio $=$

1.11

$\beta_{0}=0.1$

(outcome

prob. at

reference

$=0.52$ )

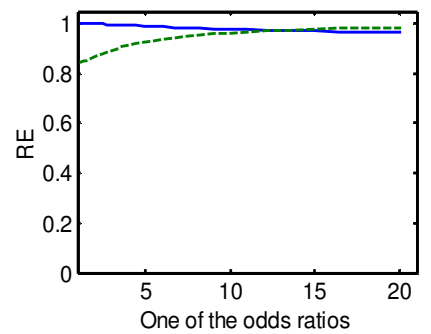

$\beta_{0}=3.0$

(outcome

prob. at

reference

$=0.95$ )

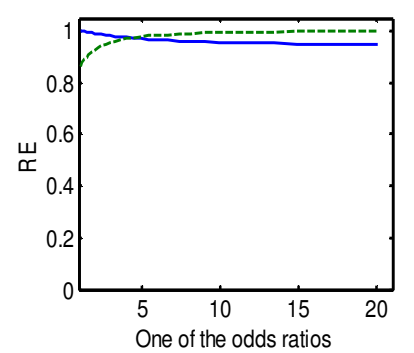

the other odds ratio $=$

2.72
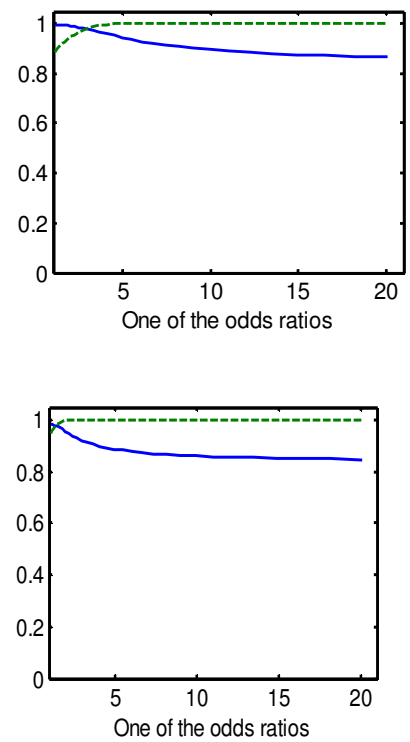

the other odds ratio $=$

7.39
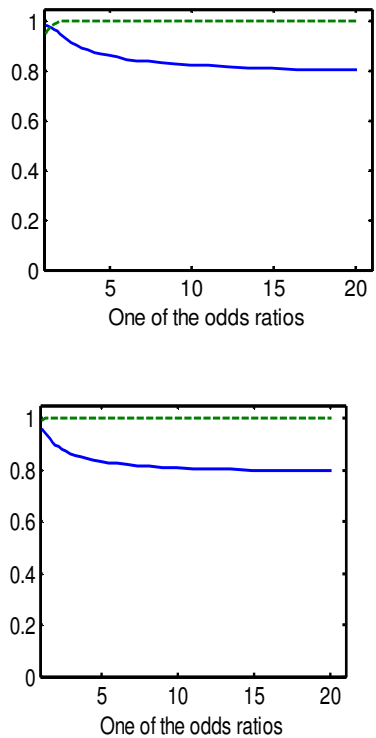

the other odds ratio $=$ 20.09
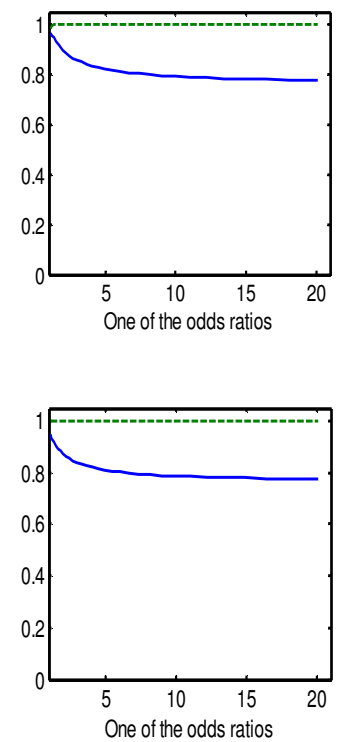

Figure 5.1. Relative efficiencies for main effects logistic model in the first class of models in Table 5.1

(solid and dashed lines for completely balanced and r-designs, respectively).

the other odds ratio $=$

1.11

$\beta_{0}=0.1$

(outcome prob. at reference $=0.52)$

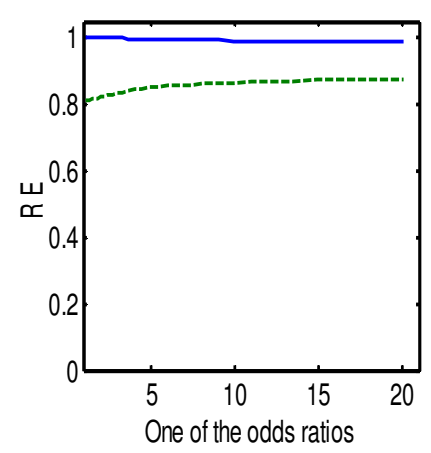

$\beta_{0}=3.0$

(outcome prob. at reference $=0.95)$ the other odds ratio $=$

2.72
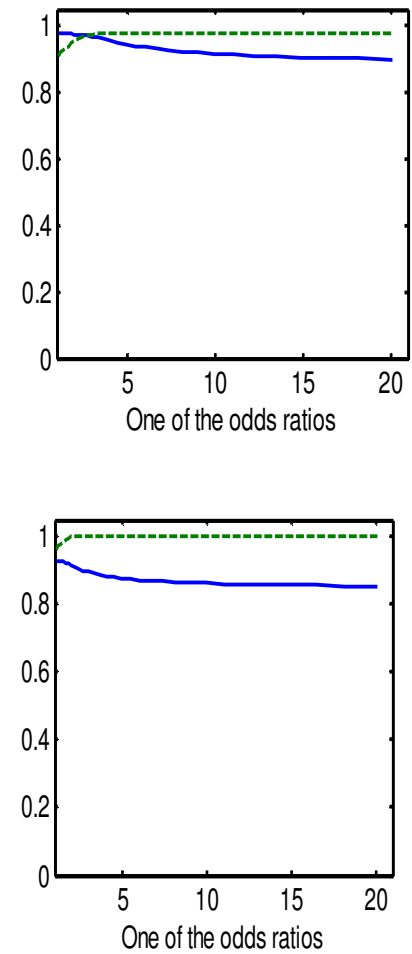

the other odds ratio $=\quad$ the other odds ratio $=$

7.39

20.09
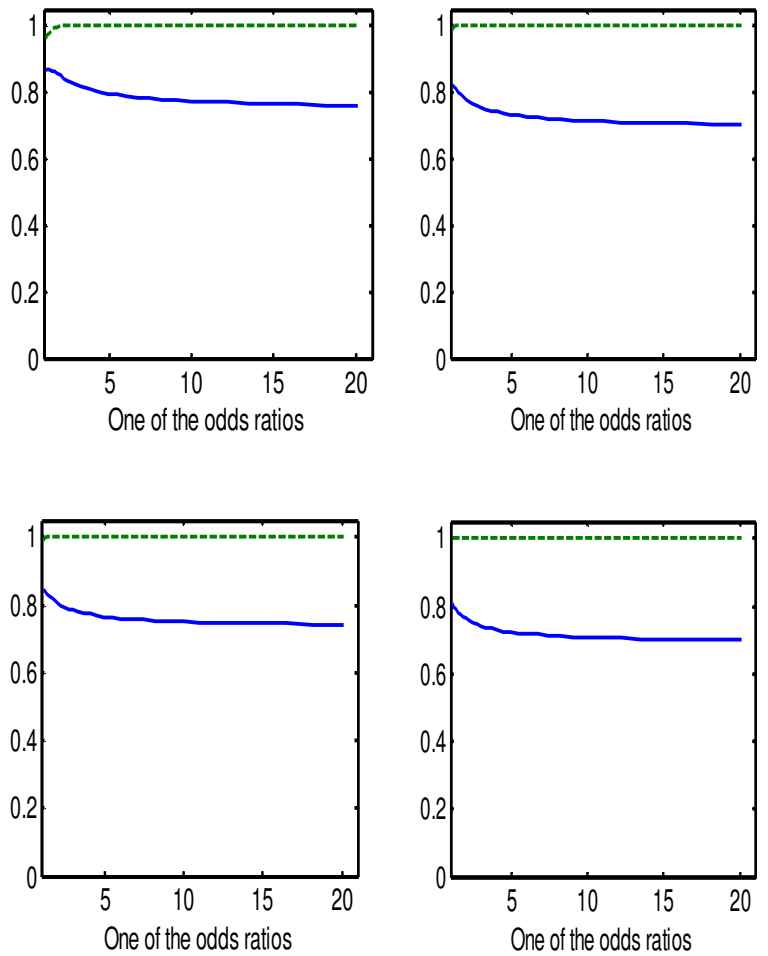

Figure 5.2. Relative efficiencies for main effects logistic model in the second class of models in Table 5.1 (solid and dashed lines for completely balanced and $\mathrm{r}$ designs, respectively). 
The values of the regression parameters for the other factors in the model are fixed at regression parameter values whose corresponding odds ratios are indicated at the top of the columns of the figures. These regression parameter values are $0.10,1.00,2.00$ and 3.00 and their corresponding odds ratios are shown at the top of the columns of the figures. These values of regression parameters are considered as a good representation of regression parameters in practice. Odds ratios are more often reported than the regression parameters found in practice. Odds ratios are more often reported than the regression parameters in the applied areas.

the other odds ratio $=$ 1.11

$\beta_{0}=0.1$

(outcome prob. at reference $=0.52$ )

$\beta_{0}=3.0$

(outcome prob. at reference $=0.95$ ) the other odds ratio $=$ 2.72
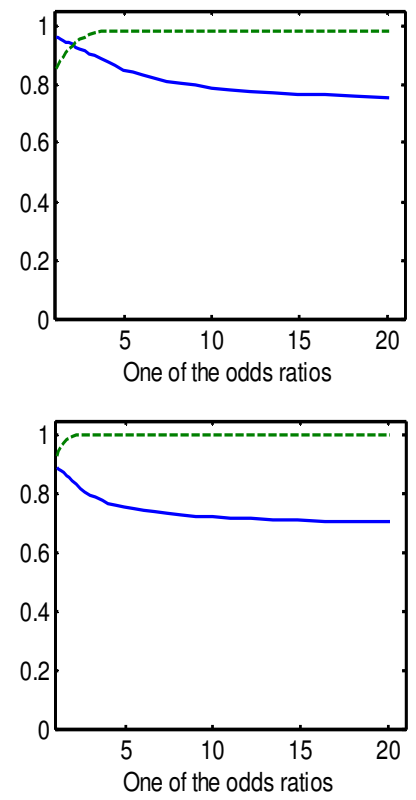

the other odds ratio $=$

7.39
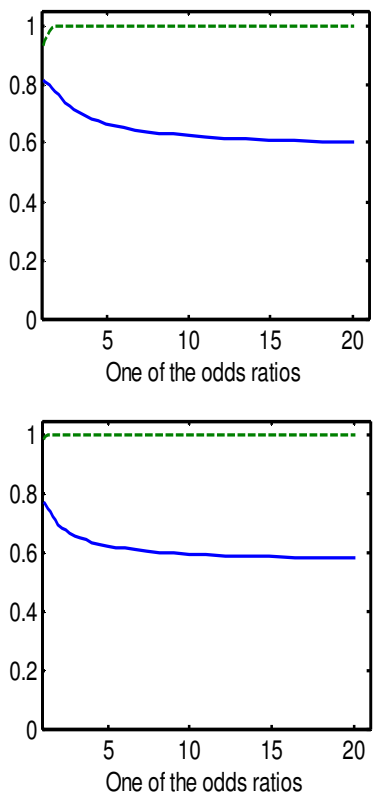

the other odds ratio $=$ 20.09
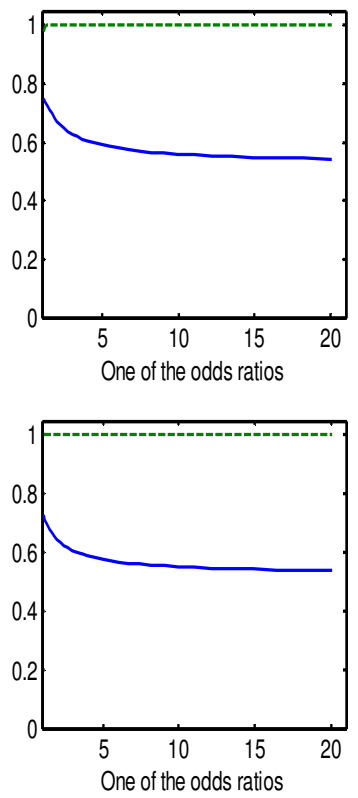

Figure 5.3. Relative efficiencies for main effects logistic model in the third class of models in Table 5.1 (solid and dashed lines for completely balanced and r-designs, respectively). 
the other odds ratio $=$

1.11

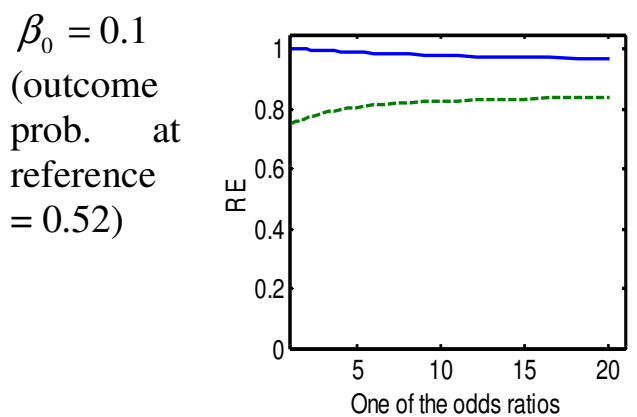

$\beta_{0}=3.0$

(outcome prob. at reference $=0.95$ ) the other odds ratio $=$

2.72
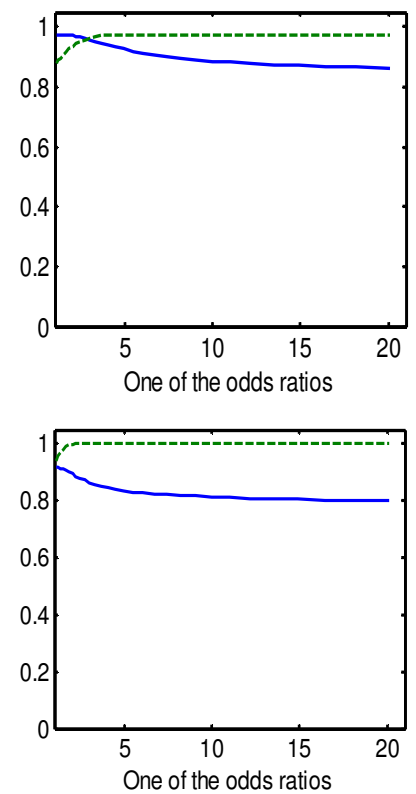

the other odds ratio $=$

7.39
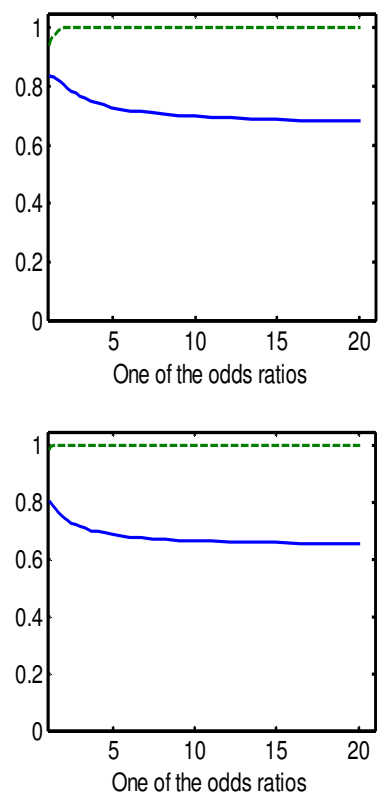

the other odds ratio $=$ 20.09
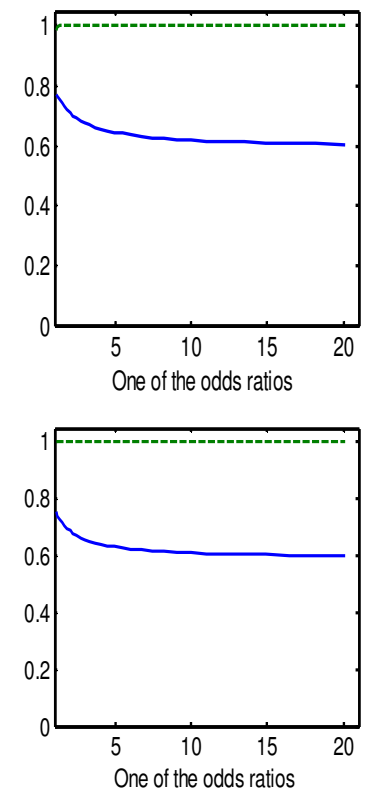

Figure 5.4. Relative efficiencies for main effects logistic model in the fifth class of models in Table 5.1 (solid and dashed lines for completely balanced and r-designs, respectively).

The results can be summarized as follow:

- The completely balanced design is more efficient than the r-design for odds ratios close to 1 (regression parameters close to 0 in absolute value).

- For odds ratios larger than 1, the r-design is more efficient than the completely balanced design. The relative efficiency for the completely balanced design drops while it increases for the r-design as the odds ratios move away from 1.

- For odds ratio values greater than (about) 3.00, the relative efficiency of the r-design is greater than that of completely balanced design and it approaches 1 as the odds ratio increases.

- For odds ratios greater than 12 the relative efficiency of the completely balanced design will drop below 0.80 for models with variables having more than 2 levels.

- The loss in efficiency for completely balanced designs will increase (RE could decline below 0.40), as the number of levels and/or variables in the model increases (see Figure $5)$. 
the other odds ratio $=$

1.11

$\beta_{0}=0.1$

(outcome

prob. at

reference

$=0.52$ )

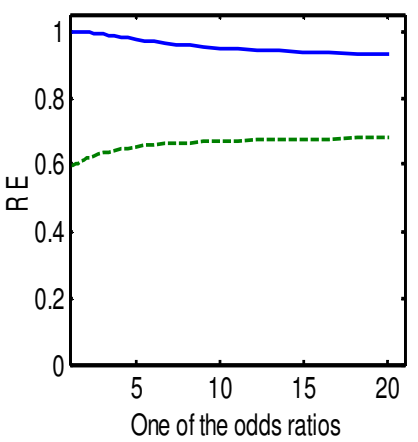

$\beta_{0}=3.0$

(outcome prob. at reference $=0.95$ )

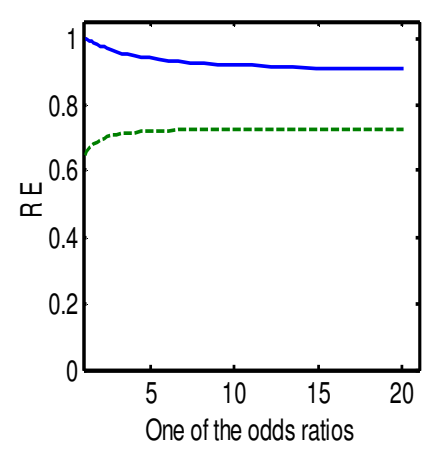

the other odds ratio $=$

2.72
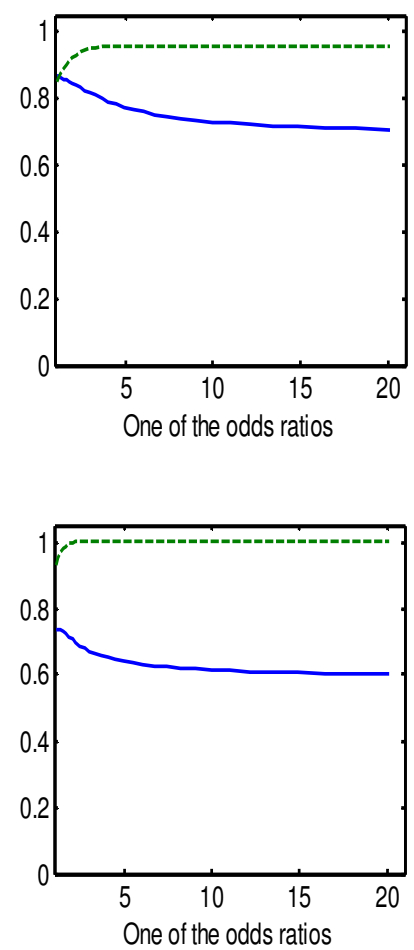

the other odds ratio $=$

7.39
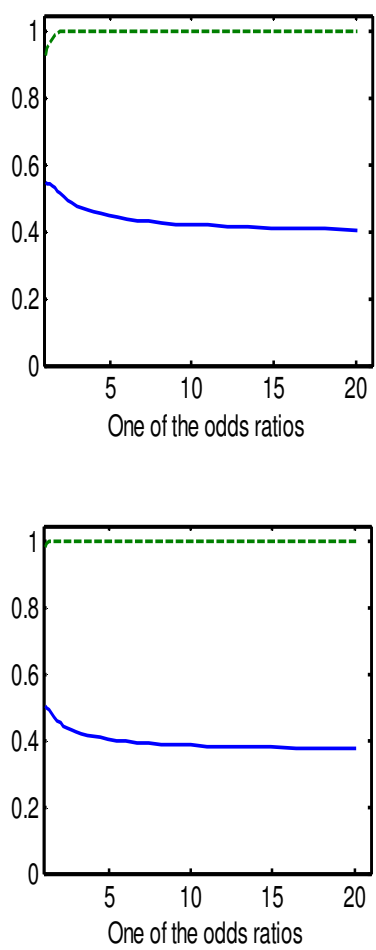

the other odds ratio $=$

20.09
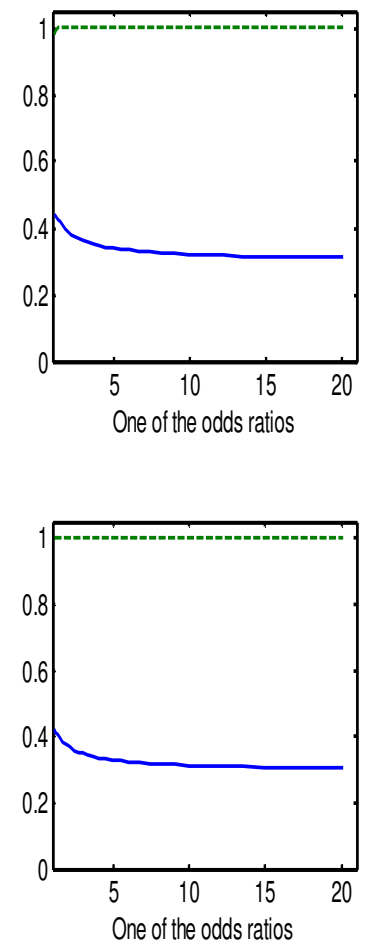

Figure 5.5. Relative efficiencies for main effects logistic model in the seventh class of models in Table 5.1 (solid and dashed lines for completely balanced and r-designs, respectively.

\section{Results for Models with Interactions}

The efficiency plots of some of the models with interaction terms are given in Figures 6-9.

The efficiency plots of the interaction models have similar pattern and that is why only some results of some models will be reported.

Similar to the Figures 1-5 in Figures 6-9, specific values of odds ratios vary across the columns and the values of the intercept parameter vary across the rows, while the relative efficiencies for the completely balanced and r-designs are shown at each plot in the cells of the figures. The value of the regression parameter for one of the factors in the model varies from 0.1 to 3.0 while the values of the other factors are fixed at $0.10,1.00,2.00$ and 3.00, respectively. 
the other odds ratios $=1.11$

$$
\begin{aligned}
& \beta_{0}=0.1 \text { (outcome } \\
& \text { probability at } \\
& \text { reference }=0.52 \text { ) }
\end{aligned}
$$

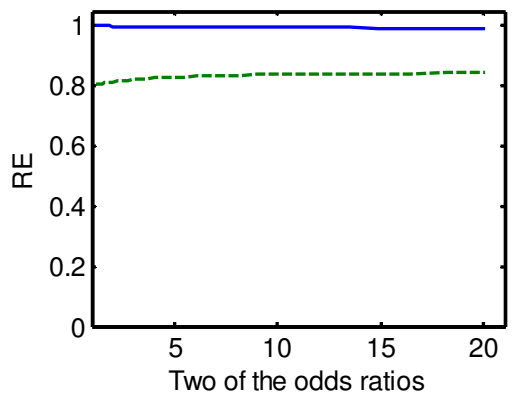

the other odds ratios $=2.72$

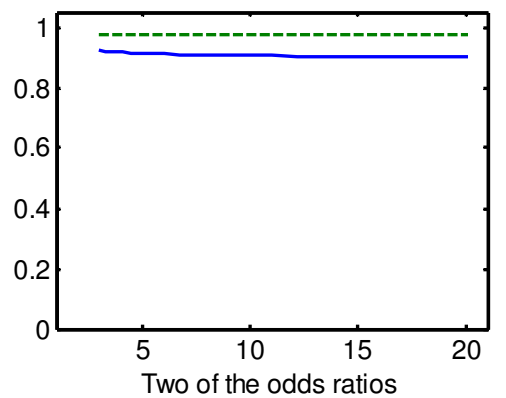

$\beta_{0}=3.0$

(outcome probability at reference $=0.92$ )
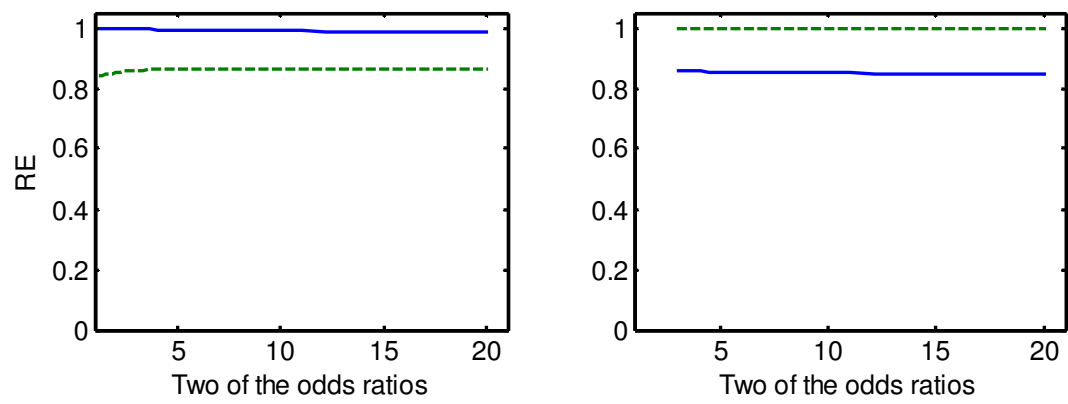

Figure 5.6. Relative efficiencies of a logistic model in the third class of models in Table 5.1 with an interaction between any two of the variables (solid and dashed lines for completely balanced and r-designs, respectively).

Because of the interaction the corresponding odds ratios are computed at cell level of the contingency tables. Thus, there are more odds ratios than the number of regression parameters in the model. The horizontal axis of the plots of Figures 6-8 shows the odds ratio values for any two cells of the contingency tables while other cells are fixed at the values given at the top of the columns of these figures. Similarly, the horizontal axis of the plots in Figure 9 shows the odds ratio values for three of the contingency table cells while the other cells are fixed at the values given at the top of the columns of the figure.

The trends of the efficiency plots are similar to that of the main effects models, i.e. as the odds ratios increase, the relative efficiency of r-designs increases while that of completely balanced designs decreases. Regardless of the values of the odds ratios and the number of levels and/or variables, the relative efficiencies of both the r-design and completely balanced design are greater than 0.80 for all models with interaction terms. The relative efficiency also increases as the number of model parameters $(p)$ becomes closer to the number of design points $(h)$ (comparison of Figures 6 and 7). 
the other odds ratios $=1.11 \& 1.22 \quad$ the other odds ratios $=2.72 \& 7.39$

$\beta_{0}=0.1$

(outcome

probability at

reference $=0.52$ )
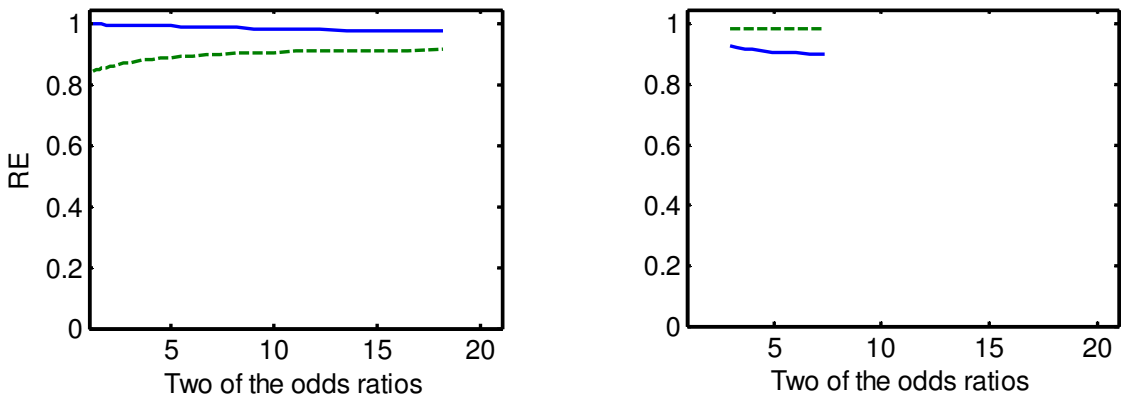

$\beta_{0}=3.0$

(outcome

probability at

reference $=0.92$ )
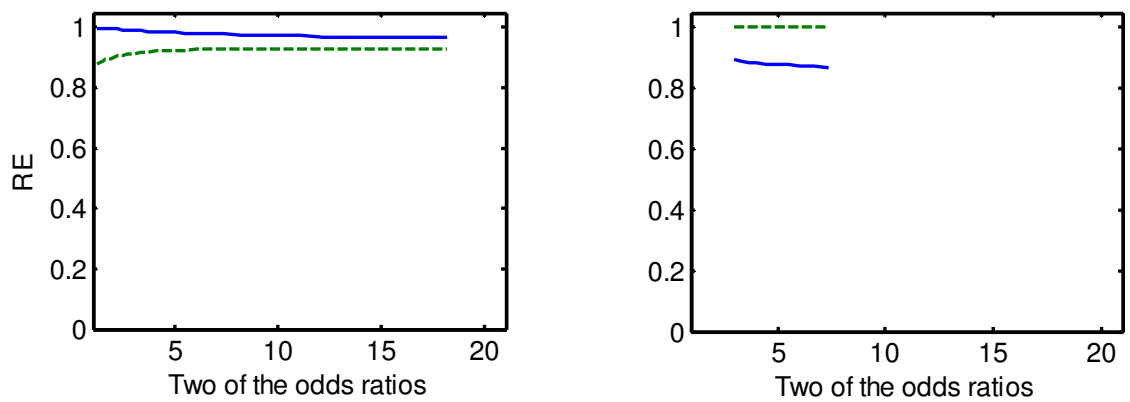

Figure 5.7. Relative efficiencies of a logistic model in the third class of models in Table 5.1 with the interaction $X_{1} X_{2}+X_{1} X_{3}$ or $X_{1} X_{2}+X_{2} X_{3}$ (solid and dashed lines for completely balanced and r-designs, respectively).

$\beta_{0}=0.1$ (outcome

probability at reference $=$ $0.52)$

the other odds ratios $=1.11 \& 1.22$ the other odds ratios $=2.72 \& 7.39$

$\beta_{0}=3.0$

(outcome probability at reference $=0.92$ )
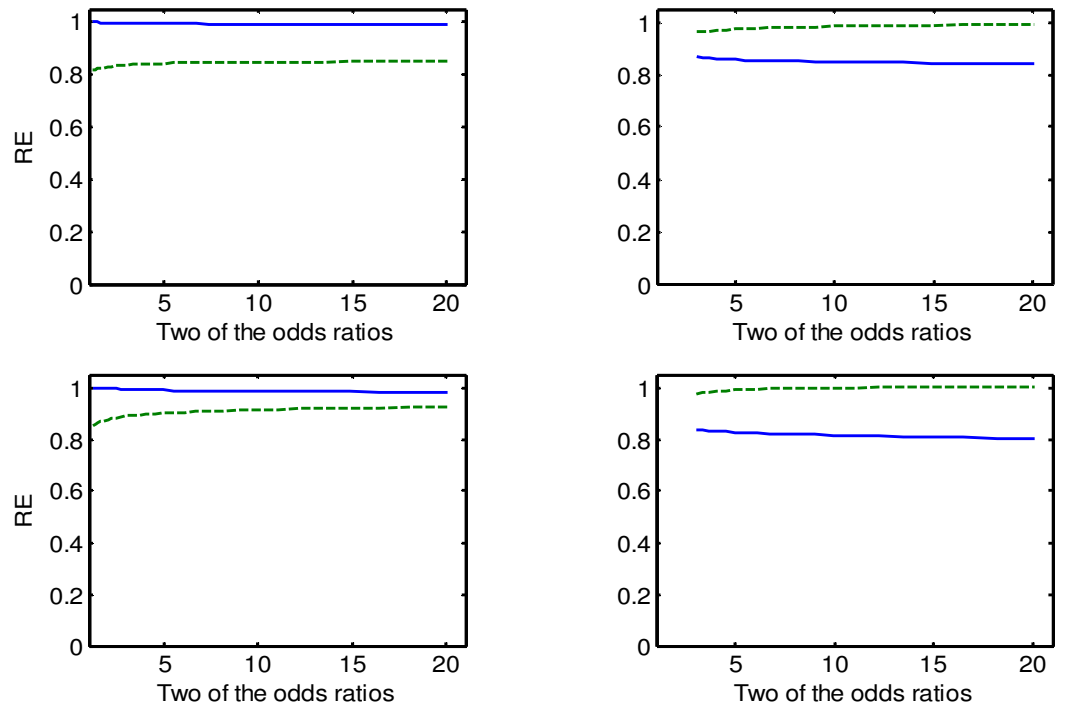

Figure 5.8. Relative efficiencies of a logistic model in the fourth class of models in Table 5.1 with the interaction $X_{1} X_{3}$ or $X_{2} X_{3}$ (solid and dashed lines for completely balanced and r-designs, respectively). 
the other odds ratios $=1.11,1.22 \& 1.35$

the other odds ratios $=2.72,7.39 \& 20.09$

$\beta_{0}=0.1$ (outcome

probability at

reference $=0.52$ )
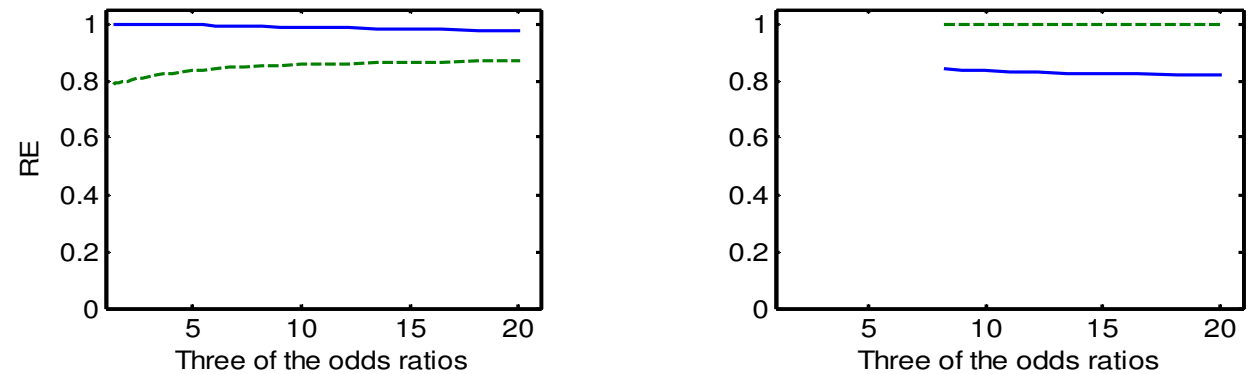

$\beta_{0}=3.0$

(outcome

probability at

reference $=0.92$ )
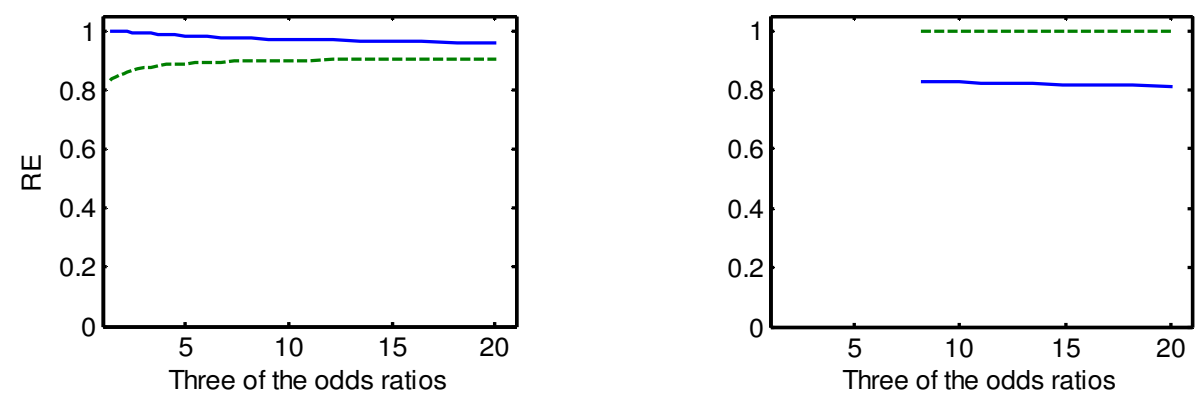

Figure 5.9. Relative efficiencies of a logistic model in the sixth class of models in Table

5.1 with all two-way interactions between the variables (solid and dashed lines for completely balanced and r-designs, respectively).

\subsection{Discussion and Conclusion}

For a saturated logistic model with categorical variables, the D-optimal, restricted Doptimal and completely balanced designs coincide. However, when the number of parameters in the model is less than the number of design points one has to make a careful design choice. Because the values of the regression parameters are not known in advance, it is difficult to find D-optimal designs. An alternative design may be the completely balanced design. The efficiency can, however, be improved by considering the r-design especially when the odds ratios are expected to be greater than about 3 . The numerical study indicates that the loss of efficiency for a completely balanced design could be large when the odds ratios are far greater than 1 and when the number of levels and/or variables in the model increases. In such cases, the relative efficiency of the r-design is close to 1 , almost as efficient as the D-optimal design. These results are based on the numerical study. A more theoretical foundation about the properties of r-designs will be the topic of future research.

Construction of the r-design requires knowledge of the rank order of the design points based on their outcome variances. This information can be supplied either by theoretical expectation of the direction of the effect of the independent variables on the outcome, or by 
information from prior studies. Misspecification of the rank of the design points will only have a negative effect on the efficiency of the design when the design points are assigned zero weight while they actually should not have zero weight or vice versa. 


\section{APPENDIX: Outline of the proof of the property}

There are several possible designs that can be selected with $p$ independent design points out of the $h$ design points in the design region $\mathbf{\Xi}$. Let $\mathbf{L}$ be the set of possible combinations of $p$ independent design points out of $h$; and $\mathbf{Z}_{l}$ be an element of $\mathbf{L}$. Then we want to minimize

$$
\left(\operatorname{Det}\left\{\mathbf{Z}_{l}\right\}\right)^{-2}\left(\prod_{k=1}^{p} w_{k} v_{k}\left(\mathbf{Z}_{l}\right)\right)^{-1}, \mathbf{Z}_{l} \in \mathbf{L}
$$

where $\mathbf{Z}_{l}$ is the $p \times p$ design matrix; and $v_{k}\left(\mathbf{Z}_{l}\right)$ is the variance of the design point which coordinates are given in the $k^{\text {th }}$ row of $\mathbf{Z}_{l}$.

For a given $\mathbf{Z}_{l},(\mathrm{~A}-1)$ is minimal when $w_{k}=\frac{1}{p}, k=1, \cdots, p$ (see lemma in the text).

Thus, A-1 will be minimized, if the product $\left(\operatorname{Det}\left\{\mathbf{Z}_{l}\right\}\right)^{2} \prod_{k=1}^{p} v_{k}\left(\mathbf{Z}_{l}\right)$ is maximized. 


\section{Maximin D-optimal Designs for Binary Longitudinal Responses}

This chapter without section 6.5 is accepted for publication in Computational Statistics and Data Analysis and available online since 8 May 2008. Authors are Tekle, F.B., Tan, F.E.S., and Berger, M.P.F. 


\begin{abstract}
Optimal design problems for logistic mixed effects models for binary longitudinal responses are considered. A function of the approximate information matrix under the framework of the Penalized Quasi Likelihood (PQL) and a generalized linear mixed model with autocorrelation is optimized. Locally D-optimal designs are computed. Maximin Doptimal designs are considered to overcome the problem of parameter value dependency of the D-optimal designs. The results show that the optimal number of repeated measurements depends on the number of regression parameters in the model. The performance of the maximin D-optimal designs in terms of the maximin efficiency (MME) is high for a range of parameter values that is common in practice. The design locations for mixed-effects logistic models generally shift to the left as compared to the design locations for general linear mixed- effects models known in the literature.
\end{abstract}

Key words: Binary responses; repeated measurements; logistic mixed-effects models; optimal designs; D-optimality; maximin D-optimality; efficiency. 


\subsection{Introduction}

Longitudinal data, often called repeated measurements in medicine and panel data in the social sciences, arise when subjects (also known as units) provide responses on multiple time/occasions. Data with this characteristic are most appropriate for studying individual change over time. A researcher designing a longitudinal study has to decide on the number and allocation of time points. Optimal design theory could help in making this decision. In optimal design theory, the optimality criteria depend on the unknown parameter values in the case of non-linear models like logistic regression and the designs are optimal locally around the given parameter values (Silvey, 1980; Atkinson and Donev, 1996). Locally optimal designs were studied by Fedorov and Müller (1997) and Melas et al (2003) among others. The parameter dependency of the optimality criteria makes the design of experiments for logistic models more complicated. Four approaches have been suggested to overcome the parameter dependency; use of an initial best guess of parameter values (Chernoff, 1953; Abdelbasit and Plackett, 1983), a sequential procedure that uses information from previous runs (Wu, 1985; Sitter and Forbes, 1997; and Sitter and Wu, 1999), a Bayesian procedure that assumes a prior distribution for the parameters (Chaloner, 1989; Chaloner and Larntz, 1989; Atkinson et al., 1993; Chaloner and Verdinelli, 1995; Dette, 1996 and Han and Chaloner, 2004), and a minimax approach which minimizes the worst possible case over a user selected region of parameters ((Müller, 1995; Dette, 1997; Müller and Pazman, 1998; Dette and Sahm, 1997, 1998; King and Wong, 2000; Imhof, 2001; Ouwens et al., 2002, 2006; Braess and Dette, 2007). Optimal designs for fixed effects logistic models in dose-response settings for clinical trials were studied in the literature using these methods (Abdelbasit and Plackett, 1983; King and Wong, 2000; Mathew and Sinha, 2001 among others). A review of optimal designs for generalized linear models (for independent observations) is given by Khuri et al. (2006). Ouwens et al., 2006 discussed balancedness of maximin designs for the logistic random intercept model with dichotomous independent variables.

Despite a wide range of literature on optimal designs for longitudinal studies with continuous responses, there is no work, to the authors' knowledge, on optimal designs of longitudinal studies with binary responses. In this chapter we will investigate the existence of designs that are robust over a range of regression parameter values for logistic mixedeffects models with repeated binary responses over time. We will first investigate locally 
D-optimal designs and then obtain numerically the maximin D-optimal designs for logistic mixed-effects models. We will also address the problem on the optimum number of repeated measurements needed for a longitudinal study with binary responses.

The Generalized Linear Models (GLMs) (McCullagh and Nelder, 1983) have been extended to unify regression methods for longitudinal data for a variety of discrete and continuous responses (Stiratelli et al, 1984; Liang and Zeger, 1986; Zeger et al., 1988; Molenberghs and Verbeke, 2005 among others). The extended version of GLMs is the Generalized Linear Mixed-effects Model (GLMM) and it has received a lot of attention in the past two decades. This chapter will focus on optimal designs for logistic mixed-effects models for correlated binary repeated measurements over time. Almost all optimality criterions in optimal design theory optimize a certain function of the information matrix. However, obtaining the information matrix presents a significant problem for GLMMs. The log-likelihood cannot be written down in closed form. Hence, either numerical methods or approximations to the log-likelihood must be used. The numerical methods are computationally intensive, even for simple models and designs. More important is that they require full knowledge of the data. This property makes numerical methods inconvenient for optimal design procedures that intend to plan on how to get the data in the best way for the estimation of parameters. For the purpose of obtaining optimal designs, we will use an approximate information matrix based on two methods that approximate the loglikelihood. We will use the Penalized Quasi-Likelihood (PQL) for logistic mixed-effects models without serial correlations (Breslow and Clayton, 1993) and an extension of GEE approach for the logistic mixed-effects models with serial correlations (Molenberghs and Verbeke, 2005). PQL for the analysis of data in linear mixed-effects models is implemented in MIWin and the SAS procedure GLIMMIX among others.

The chapter is organized as follows. The next section discusses the general logistic mixed-effects model and the variance-covariance matrix of parameter estimators. The optimality criterion and relative efficiency as a measure of performance of a design are discussed in section 6.3. A numerical study and its results are given in section 6.4. A practical example is considered in section 6.5. A software program that helps to obtain locally D-optimal and maximin designs for mixed-effects logistic models will be described in section 6.6. Discussion and conclusions are given in section 6.7. 


\subsection{Logistic mixed-effects models and optimal designs}

\subsubsection{General logistic mixed-effects models}

Let the $q \times 1$ vector $\mathbf{y}_{i}=\left(y_{i 1}, \cdots, y_{i q}\right)^{\prime}$ be the binary response vector of $q$ responses of subject $i, i=1, \cdots, N$. It is assumed that, conditionally on $r$-dimensional random effects $\mathbf{b}_{i}$ that are assumed to be drawn independently from the multinormal distribution with mean zero and covariance $\mathbf{G}$, the binary responses $y_{i j}, j=1, \cdots, q$ are independently Bernoulli distributed with probability of success $p\left(y_{i j} \mid \mathbf{b}_{i}\right)$. These probabilities are related to the fixed and random effects via the logit link and the corresponding logistic mixed-effects model is given by:

$$
\operatorname{logit}\left(p\left(y_{i j} \mid b_{i}\right)\right)=\mathbf{x}_{j}^{\prime} \boldsymbol{\beta}+\mathbf{z}_{j}^{\prime} \mathbf{b}_{i},
$$

where the $p \times 1$ vector $\mathbf{x}_{j}$ is the design vector of the explanatory variables at the $j^{\text {th }}$ measurement for subject $i, \mathbf{z}_{j}$ is the $r \times 1$ vector that may be a subset of the design vector $\mathbf{x}_{j}$, and the $p \times 1$ vector $\boldsymbol{\beta}$ is a vector of $p$ fixed regression coefficients.

For simplicity, we will assume that subjects will have measurements at the same time points and all the subjects will have complete data. We also assume that the vector $\mathbf{x}_{j}$ only consists of the polynomial terms of time $t_{j}$ at the $j^{\text {th }}$ measurement for $N$ subjects. For instance, if a linear predictor is assumed, then the design vector is $\mathbf{x}_{j}^{\prime}=\left(\begin{array}{ll}1 & t_{j}\end{array}\right)$. Suppose that $\mathbf{x}_{j}^{\prime}=\mathbf{z}_{j}^{\prime}=\left(\begin{array}{ll}1 & t_{j}\end{array}\right), \boldsymbol{\beta}=\left(\beta_{0} \beta_{1}\right)^{\prime}$, and $\mathbf{b}_{i}=\left(b_{0 i} b_{1 i}\right)^{\prime}$, where $\beta_{0}$ and $\beta_{1}$ are the intercept and the slope parameters for the fixed effects, $b_{0 i}$ and $b_{1 i}$ are the intercept and the slope parameters for the random effects. Then, according to the logistic mixed-effects model given by (6.1), the log-odds of a success response for subject $i$ at time $t_{j}$ is the linear function of time, $\operatorname{logit}\left(p\left(y_{i j} \mid b_{i}\right)\right)=\left(\beta_{0}+b_{0 i}\right)+\left(\beta_{1}+b_{1 i}\right) t_{j}$. The objective is to identify optimal locations of time points $t_{j}(j=1, \cdots, q)$ in such a way that the parameters $\boldsymbol{\beta}=\left(\beta_{0} \beta_{1}\right)^{\prime}$ are estimated as efficient as possible. In addition to the optimal allocations of time points, the number of time points $q$ could also be optimally determined. Obviously, $q \geq p$ so that the parameters in $\boldsymbol{\beta}$ are estimable. 


\subsection{2. variance-covariance matrix of the parameter estimators}

Due to the intractable form of the log-likelihood function for model (6.1), different approaches for approximation of the log-likelihood have been presented in literature. The asymptotic variance-covariance matrix of $\hat{\boldsymbol{\beta}}$, which is the inverse of the information matrix of the parameters based on both the methods of penalized quasi-likelihood (PQL) (Breslow and Clayton, 1993) and the extension of the GEE approach for mixed-effects models (Molenberghs and Verbeke, 2005) will be considered for the optimal-design procedure.

\section{PQL method for logistic mixed-effects models without serial correlations}

Breslow and Clayton (1993) used Laplace methods for the integral approximation of the log-likelihood function of the data and obtained PQL. They defined a working response vector in such a way that an iterative weighted least-squares algorithm can be applied to estimate the fixed effects and then the random effects parameters (see Breslow and Clayton (1993) for details). A logistic mixed-effects model without serial correlations is considered in the PQL method. An approximate variance-covariance matrix for the parameters estimator of $\boldsymbol{\beta}$ at the end of the iterative steps of the PQL method is given by:

$$
\operatorname{var}(\hat{\boldsymbol{\beta}}) \approx\left(\mathbf{X}^{\prime} \mathbf{V}^{-1} \mathbf{X}\right)^{-1}
$$

where $\hat{\boldsymbol{\beta}}$ is the estimator of $\boldsymbol{\beta}$ for logistic mixed-effects model in (6.1), the $N q \times p$ matrix $\mathbf{X}$ is formed by stacking of $\left\{\mathbf{x}_{j}^{\prime}\right\}$ for $N$ subjects, and the $N q \times N q$ matrix $\mathbf{V}$ is the blockdiagonal matrix with blocks $q \times q$ matrices given by:

$$
\mathbf{v}_{i} \approx \mathbf{w}_{i}^{-1}+\mathbf{z G z}^{\prime}
$$

where the $q \times r$ matrix $\mathbf{z}$, whose rows are $\mathbf{z}_{j}^{\prime}, j=1, \cdots q$, the $r \times r$ matrix $\mathbf{G}$ is the variance-covariance matrix of the random effects, $\mathbf{w}_{i}$ is a diagonal matrix of the conditional variances of the responses given the random effects $\mathbf{b}_{i}$, i.e.

$$
\mathbf{w}_{i}=\operatorname{diag}\left(w_{i 1}^{\mathbf{b}_{i}}, \cdots, w_{i q}^{b_{i}}\right),
$$

where $w_{i j}^{\mathbf{b}_{i}}=\operatorname{var}\left(y_{i j} \mid \mathbf{b}_{i}\right)=v\left(p\left(y_{i j} \mid \mathbf{b}_{i}\right)\right)$, for $i=1, \cdots, N, j=1, \cdots q, v(\cdot)$ is a variance function for binary response (McCullagh and Nelder, 1989). We will generate $\mathbf{b}_{i}$ from a multivariate normal distribution with mean zero and covariance $\mathbf{G}$ and $p\left(y_{i j} \mid \mathbf{b}_{i}\right)$ will be obtained using (6.1). 


\section{Extension of GEE method for logistic mixed-effects models with serial correlations}

Zeger et al. (1988) and Molenberghs and Verbeke (2005) have extended the generalized estimating equation (GEE) approach (Zeger et. al., 1986) to include serial correlations of repeated measurements in the standard formulation of generalized linear mixed-effects models (GLMM). The approximate variance-covariance matrix of the parameter estimators is:

$$
\operatorname{var}(\hat{\boldsymbol{\beta}}) \approx\left(\sum_{i=1}^{N} \frac{\partial \mathbf{p}_{i}^{\prime}}{\partial \boldsymbol{\beta}} \mathbf{v}_{i}^{-1} \frac{\partial \mathbf{p}_{i}}{\partial \boldsymbol{\beta}^{\prime}}\right)^{-1},
$$

where $\hat{\boldsymbol{\beta}}$ is the estimator of $\boldsymbol{\beta}, \mathbf{p}_{i}=\left(p\left(y_{i 1} \mid \mathbf{b}_{i}\right), \cdots, p\left(y_{i q} \mid \mathbf{b}_{i}\right)\right)^{\prime}, \frac{\partial \mathbf{p}_{i}^{\prime}}{\partial \boldsymbol{\beta}}=\mathbf{x}^{\prime} \mathbf{w}_{i}$, the $q \times p$ matrix $\mathbf{x}$ with rows $\mathbf{x}_{j}^{\prime}$, for $j=1, \cdots q$, and the working variance-covariance of the responses $\mathbf{v}_{i}$ is given by:

$$
\mathbf{v}_{i} \approx \mathbf{w}_{i}^{1 / 2} \mathbf{R}(\rho) \mathbf{w}_{i}^{1 / 2}+\mathbf{w}_{i} \mathbf{z G} \mathbf{z}^{\prime} \mathbf{w}_{i}^{\prime},
$$

where $\mathbf{w}_{i}$ is the diagonal matrix of the conditional variances of the responses given the random effect $\mathbf{b}_{i}=0$, i. e.

$$
\mathbf{w}_{i}=\operatorname{diag}\left(w_{i 1}^{0}, \cdots, w_{i q}^{0}\right),
$$

where $w_{i j}^{0}=\operatorname{var}\left(y_{i j} \mid \mathbf{b}_{i}=\mathbf{0}\right)=v\left(p\left(y_{i j} \mid \mathbf{b}_{i}=\mathbf{0}\right)\right)$, for $i=1, \cdots, N, j=1, \cdots q, \quad v(\cdot) \quad$ is a variance function for binary response (McCullagh and Nelder, 1989). The $q \times q$ matrix $\mathbf{R}(\rho)$ is the correlation matrix of the responses over time (see section 24.2.3 in Molenberghs and Verbeke (2005) for details). An autoregressive correlation structure of order $1(\mathrm{AR}(1))$ is considered in this chapter for the correlation matrix $\mathbf{R}(\rho)$, i.e. the $\left(j, j^{\prime}\right)$ element of the correlation matrix $\mathbf{R}(\rho)$ is equal to $\rho^{\left|t_{j}-t_{j}\right|}$, where $\rho$ is the autocorrelation coefficient that gives the correlation between two time points that are one unit of time apart. This correlation structure implies that repeated measurements closer in time are more highly correlated and that the correlation decreases as the difference in time points increases.

The general approach to optimal design is to search a design that optimizes a specified function of the asymptotic variance-covariance matrix of the parameter estimators. 


\subsubsection{Optimal designs and relative efficiency}

\section{Locally D-optimal designs and relative efficiency}

Let the design space of $q \times 1$ time vectors be denoted by $\Xi$, i.e. $\Xi=\left\{\left(t_{1}, \cdots, t_{q}\right)^{\prime}: t_{j} \in[a, b]\right\}$. A design $\xi_{q}$ is an element of the design space $\Xi$ with all possible designs having $q$ repeated measurements at distinct time points for all $N$ subjects. The amount of information in a design is measured by the Fisher information matrix, which is equal to the inverse of the asymptotic variance-covariance matrix of the parameter estimators in (6.2) and/or (6.5). The optimal design $\xi_{q}^{*}$ is the design among all possible designs $\xi_{q}$ with $q$ time points for which a function of the covariance matrix is minimized. The most commonly used function of the covariance matrix is the determinant function and the corresponding optimality criterion is known as the D-optimality criterion. We will denote a D-optimality criterion for the $\xi_{q}$ designs by $\mathrm{D}_{q}$. The $\mathrm{D}_{q}$-optimal design minimizes the determinant of the variance-covariance matrix (Tan and Berger, 1999). The determinant depends on the unknown vector of regression parameters $\boldsymbol{\beta}$, the covariance parameters for random effects in $\mathbf{G}$, the correlation parameters in $\mathbf{R}$, and the design $\boldsymbol{\xi}_{q}$. Thus, given a vector of regression parameters $\boldsymbol{\beta}$ and the parameters in $\mathbf{G}$ and $\mathbf{R}$, the determinant of the variance-covariance matrix is minimized for a locally D-optimal design $\xi_{q}^{*} \in \Xi$, i.e.:

$$
\operatorname{Det}\left[\operatorname{var}\left(\hat{\boldsymbol{\beta}}_{\xi_{q}^{*}}\right)\right] \leq \operatorname{Det}\left[\operatorname{var}\left(\hat{\boldsymbol{\beta}}_{\xi_{q}}\right)\right],
$$

for each design $\xi_{q} \in \Xi$, where $\operatorname{var}\left(\hat{\boldsymbol{\beta}}_{\xi_{q}^{*}}\right)$ and $\operatorname{var}\left(\hat{\boldsymbol{\beta}}_{\xi_{q}}\right)$ are the variance-covariance matrices of the parameter estimators under $\xi_{q}^{*}$ and $\xi_{q}$, respectively.

We will restrict ourselves to this criterion as it has some nice properties. The D-optimal design minimizes the volume of the asymptotic confidence ellipsoid of the parameter estimators. $\mathrm{D}_{q}$-optimal designs also have a nice property that they do not depend on the chosen time interval $[a, b]$, where $a$ and $b$ are the two end points of the time interval, i.e., if a linear transformation is considered on the time interval, the D-optimal designs can be obtained for the new time interval by applying the same transformation (Ouwens et al., 2006).

Relative efficiency can be used as a measure of comparison for two designs. To fairly compare designs with a different number of time points, we have fixed the total number of 
measurements $M=N q$. The number of subjects $N$ and the number of repeated measurements over time $q$ may vary in the comparison. Let $\xi_{q}$ be a design with number of time points $q \geq p$, where $p$ is the number of regression parameters in the model. Then the relative efficiency (RE) of $\xi_{q}$ compared to $\xi_{p}$ is given by:

$$
\mathrm{RE}_{\xi_{q} \mid \xi_{p}}=\frac{p}{q}\left[\frac{\operatorname{Det}\left[\operatorname{var}\left(\hat{\boldsymbol{\beta}}_{\xi_{p}}\right)\right]}{\operatorname{Det}\left[\operatorname{var}\left(\hat{\boldsymbol{\beta}}_{\xi_{q}}\right)\right]}\right]^{1 / p},
$$

where $\operatorname{var}\left(\hat{\boldsymbol{\beta}}_{\xi_{p}}\right)$ and $\operatorname{var}\left(\hat{\boldsymbol{\beta}}_{\xi_{q}}\right)$ are the variances of the estimators of the regression vector $\boldsymbol{\beta}$ under the designs $\xi_{p}$ and $\xi_{q}$, respectively.

The reciprocal of the $\mathrm{RE}_{\xi_{q} \xi_{p}}$ is equal to the relative amount of extra observations that must be taken under $\xi_{q}$ to obtain the same efficiency as under the $\xi_{p}$ (Atkinson and Donev (1996)). For example, if $\mathrm{RE}_{\xi_{q} \mid \xi_{p}}=0.80$, then $(1 / 0.80-1) \times 100 \%=25 \%$ more observations will be needed under design $\xi_{q}$ to obtain the same efficiency as under design $\xi_{p}$

Note that the RE in (6.9) compares a design for more number of time points with a saturated design, which has the number of time points equal to the number of regression parameters in the model. However, the saturated design may not always be the optimal design and it is arbitrarily selected as a reference design to compare the designs.

\section{Maximin D-optimal designs}

The D-optimality criterion in (6.8) depends on the regression parameter $\boldsymbol{\beta}$ through the variance functions for logistic models. Since the exact values of $\boldsymbol{\beta}$ are unknown in practice, we consider a maximin D-optimal design criterion that does not depend on $\boldsymbol{\beta}$. The maximin design criterion requires a specification of a region for the regression parameters in the model (Ouwens et al., 2002; Braess and Dette, 2007). We assume that $\boldsymbol{\beta} \in \mathrm{B}=\left[\beta_{\min }, \beta_{\max }\right]^{p} \in \mathfrak{R}^{p}$ and find an optimal design within the specified region of the

parameter space. A design $\xi_{q}^{\text {maximin }} \in \Xi$ is maximin D-optimal if it maximizes the smallest relative efficiency over $\Xi$ (Ouwens et al.(2002)). The maximin D-optimal design maximizes the minimum gain in relative efficiency among all designs with $q$ time points and the corresponding maximin efficiency is given by: 


$$
\mathrm{MME}=\max _{\xi_{\mathrm{q}} \in \Xi}\left\{\min _{\mathrm{B}}\left[\operatorname{RE}\left(\xi_{\mathrm{q}}, \xi^{*} \mathrm{q}\right)\right]\right\}
$$

where for each vector of regression parameter $\boldsymbol{\beta} \in \mathrm{B}$, the relative efficiency $\mathrm{RE}_{\xi_{q} \mid \xi_{q}^{*}}$ is computed with respect to the locally D-optimal design $\xi_{q}^{*}$ for that particular vector of regression parameter.

In section 4 we will consider a numerical study to obtain locally D-optimal designs and identify the maximin D-optimal designs.

\section{Optimal number of time points}

The number of time points at which measurements are taken for a longitudinal study should be restricted in practice because of logistical and ethical reasons. In most practical situations the number of time points is decided on a more or less ad-hoc basis. We compare maximin D-optimal designs with different number of time points and identify the most efficient design. First, the maximin D-optimal designs with a given number of time points are identified and then these designs with different number of time points are compared using the relative efficiency given in Equation (6.9). Let $\xi_{q}^{\operatorname{maximin}}$ be the maximin Doptimal design with number of time points $q \geq p$. The relative efficiency of a maximin D-

optimal design $\xi_{q}^{\text {maximin }}$ compared to a maximin D-optimal design $\xi_{p}^{\text {maximin }}, \mathrm{RE}_{\xi_{q}^{\text {maximin } \mid \xi_{p}^{\max m i n}}}$, can be computed using Equation (6.9). In computing $\operatorname{RE}\left(\xi_{q}^{\operatorname{maximin}}, \xi_{p}^{\operatorname{maximin}}\right)$, the ratio of the determinants in Equation (6.9) are obtained in such a way that the maximin D-optimal designs are $\xi_{p}^{\text {maximin }}$ and $\xi_{q}^{\text {maximin }}$ over the parameter values $\boldsymbol{\beta} \in \mathrm{B}$, where $\mathrm{B}$ is the parameter space.

\subsection{Numerical study and Results}

\subsubsection{Models and parameter values}

We have considered the time interval $[-1,1]$ for the numerical study in the search for optimal allocations of time points. Many large-scale longitudinal studies often measure the subjects over more than 5 to 10 time points. Therefore, we restricted the number of repeated measurements to $q \leq 15$. Since the patterns of the allocation of time points and $\mathrm{RE}$ are similar for different values of the number of time points $q$, we will present the 
results only for $q \leq 5$.

Optimal allocations of time points and number of repeated measurements are numerically obtained for random intercept and random slope logistic models with linear, quadratic and cubic terms for the predictor. The two variance-covariance matrices discussed in section 2.2 under PQL and extended GEE approaches are considered for the optimization procedure. First, locally D-optimal designs are computed for a given combination parameter values. Then, maximin D-optimal designs that are optimal over an interval of the regression parameters are considered. It is known that the performance and results of maximin D-optimal designs depend on the range of the parameter spaces (Ouwens et al., 2002; Braess and Dette, 2007). We have selected a range of interval (0.0, $1.5)$ with the maximum odds ratio is $20(\exp (2 * 1.5))$ as a quick review of recently published medical and health science researches shows that the odds ratios are below 20 in most cases. We have also considered a wider $(0.0,3.0]$ with the maximum odds ratio 403 $(\exp (2 * 3))$ to compare the performance of maximin D-optimal designs for wider and narrow intervals.

As described in section 2.2, the PQL method needs the random effects $\mathbf{b}_{i}$ in the iteration steps based on the full data and parameter estimators. We have generated the values of $\mathbf{b}_{i}$ from a multivariate normal distribution with mean zero and variancecovariance $\mathbf{G}$. The following cases are considered for the covariance parameters in $\mathbf{G}$ :

- $\mathbf{G}_{1}=\left[\begin{array}{ll}1 & 0 \\ 0 & 0\end{array}\right]$, i.e., a random intercept model with variance of the random intercept $g_{11}$, which can be set to $g_{11}=1$ without loss of generality.

- $\mathbf{G}_{2}=\left[\begin{array}{cc}1 & 0 \\ 0 & 0.25\end{array}\right]$, a random intercept and random slope model with variances for the random intercept $g_{11}=1$ and random slope $g_{22}=0.25$. The correlation between random intercept and random slope is assumed zero.

- $\mathbf{G}_{3}=\left[\begin{array}{ll}1 & 0.5 \sqrt{0.25} \\ 0.5 \sqrt{0.25} & 0.25\end{array}\right]$, a random intercept and random slope model with correlation between the random intercept and random slope $r_{12}=0.5$. Note that the covariance between random intercept and random slope $g_{12}=r_{12} \sqrt{g_{11} g_{22}}$.

We have made a simulation study and observed that the variance-covariance matrix of the parameter estimators becomes stable as the number of subjects is greater than 500 . The 
variance of the determinant of the variance-covariance matrix of the parameter estimators for different simulated studies with random effects are generated from a multivariate normal distribution for each subject is almost zero when the number of subjects is $N=500$ (Figure 6.1). Therefore, we have generated the random effects $\mathbf{b}_{i}$ for 500 subjects to obtain the optimal designs under the PQL method. Note that for the extended GEE method, generation of the random effects is not required as the random effects $\mathbf{b}_{i}$ are approximated by the mean of the distribution, i.e., $\mathbf{b}_{i}=\mathbf{0}$, for $i=1, \cdots, N$. A matlab code written to obtain results in this chapter can be obtained from the authors upon request.

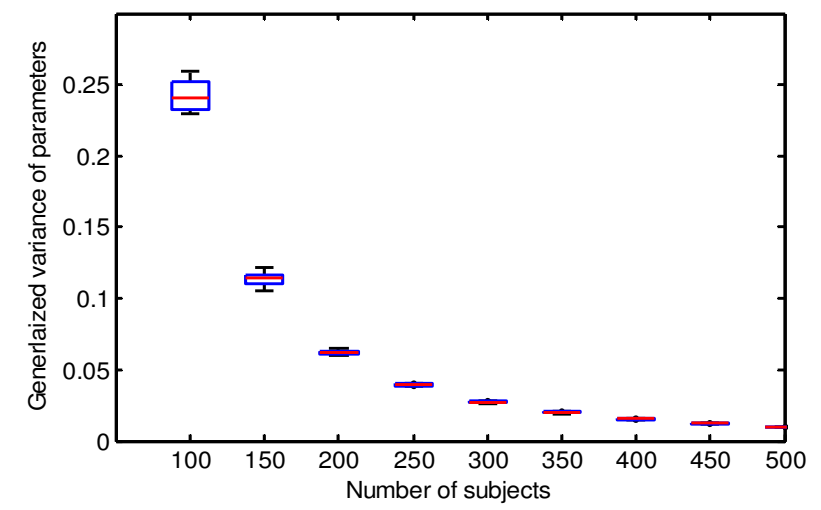

Figure 6.1. Box plots that show the variability of the generalized variance from 10 different simulated studies.

\subsubsection{Results}

\section{Locally D-optimal allocations of time points}

Figure 6.2 shows the optimal allocations of time points at the regression parameter values for logistic mixed-effects models. The optimal allocations of time points for random intercept logistic models are approximately the same as that of random intercept and slope logistic models. Hence, results are shown in Figure 6.2 only for the random intercept logistic models.

In the plots in Figure 6.2, the number of time points $q$ varies across the rows and the anticipated method of estimation varies across the columns. The optimal allocations of time points for logistic mixed-effects models based on the PQL method are shown in the first column while results for logistic mixed-effects models with serial correlations (based on extended version of GEE method) are shown in the last two columns. In each plot, the vertical axis shows the slope parameter values and the horizontal axis shows the locations of the time points. 
As it can be observed from Figure 6.2, the optimal location of time points include the two end points of the time interval when the values of regression parameter is small and the time points move away from the two end points as the value of the parameter increases. Chipman and Welch (1996) have found a similar phenomenon in their comparison of Doptimal designs for generalized linear models. They show that the optimal design points for the logistic models 'move in' from the edge of the design region. The non-linear relation between the response probability and the predictor causes this move. The results in Figure 6.2 confirm that the phenomenon is also true for logistic mixed-effects models.

Silvey (1980, p.59) and Sebastiani and Settimi (1997) among others showed for a fixed effects logistic model that a design with two design points, $t_{1}^{*}$ and $t_{2}^{*}$, is D-optimal if the interval for the design variable is in $[\mathrm{a}, \mathrm{b}]$ and $p\left(y_{a}\right) \leq p\left(y_{t_{1}^{*}}\right)<p\left(y_{t_{2}^{*}}\right) \leq p\left(y_{b}\right)$ such that probability of success at $t_{1}^{*}$ and $t_{2}^{*}$ are, respectively, $p\left(y_{t_{1}^{*}}\right) \approx 0.176$ and $p\left(y_{t_{2}^{*}}\right) \approx 0.824$. $p\left(y_{a}\right)$ and $p\left(y_{b}\right)$ are probabilities of success at the two end points of the time intervals a and $b$, respectively. Based on the numerical study, three cases are observed for the Doptimal allocations of time points for a mixed-effects logistic model with 2 time points. First, the optimal time points are at $t_{1}^{*}$ and $t_{2}^{*}$ at which the marginal probabilities of success are $p\left(y_{t_{1}^{*}}\right) \approx 0.154$ and $p\left(y_{t_{2}^{*}}\right) \approx 0.846$, if $p\left(y_{-1}\right) \leq p\left(y_{t_{1}^{*}}\right)<p\left(y_{t_{2}^{*}}\right) \leq p\left(y_{1}\right)$, where $p\left(y_{t}\right)$ is the marginal probability of success at time point $t$, which is the average probability over all subjects at time point $t$. The second case is that when $p\left(y_{t_{1}^{*}}\right)<p\left(y_{-1}\right)$ or $p\left(y_{t_{2}^{*}}\right)>p\left(y_{1}\right)$, then the optimal time points are at $t_{1}^{*}=-1$ and $0<t_{2}^{*}<1$ such that $p\left(y_{t_{2}^{*}}\right) \approx 0.846$ or $-1<t_{1}^{*}<0$ and $t_{2}^{*}=1$ such that $p\left(y_{t_{1}^{*}}\right) \approx 0.154$. In the third case, the optimal designs are at the two end points -1 and 1 if $p\left(y_{t_{1}^{*}}\right)<p\left(y_{-1}\right)<p\left(y_{1}\right)<p\left(y_{t_{2}^{*}}\right)$. 
Number of time points

$q=2$

$q=3$

$q=4$

$q=5$
PQL method
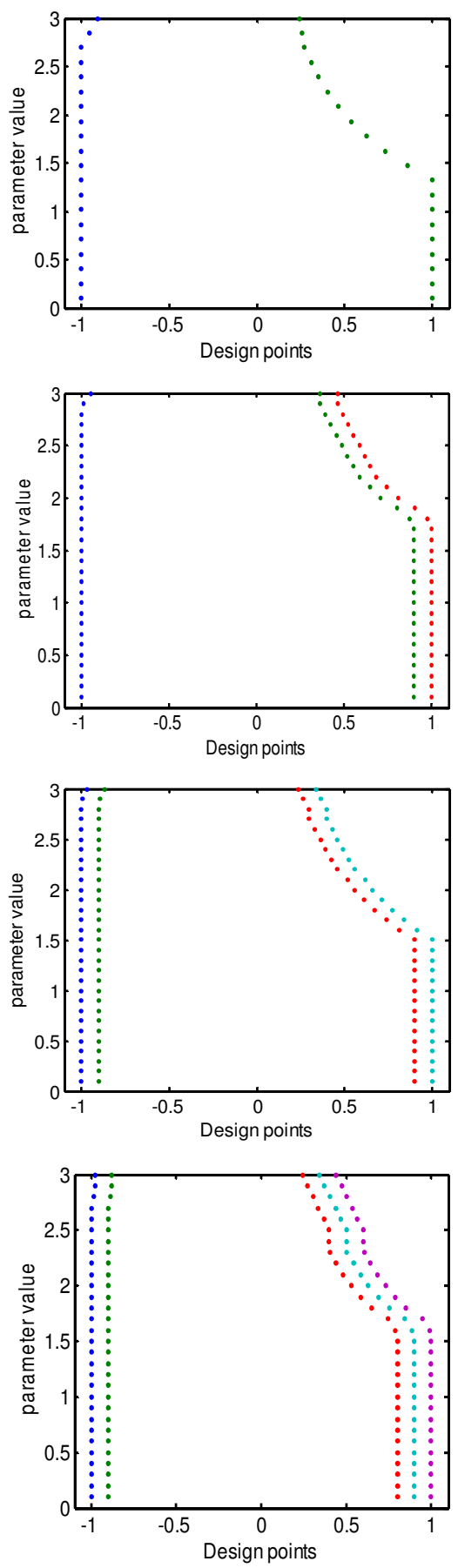

Extended GEE method with AR(1) correlation structure $\rho=0.5$
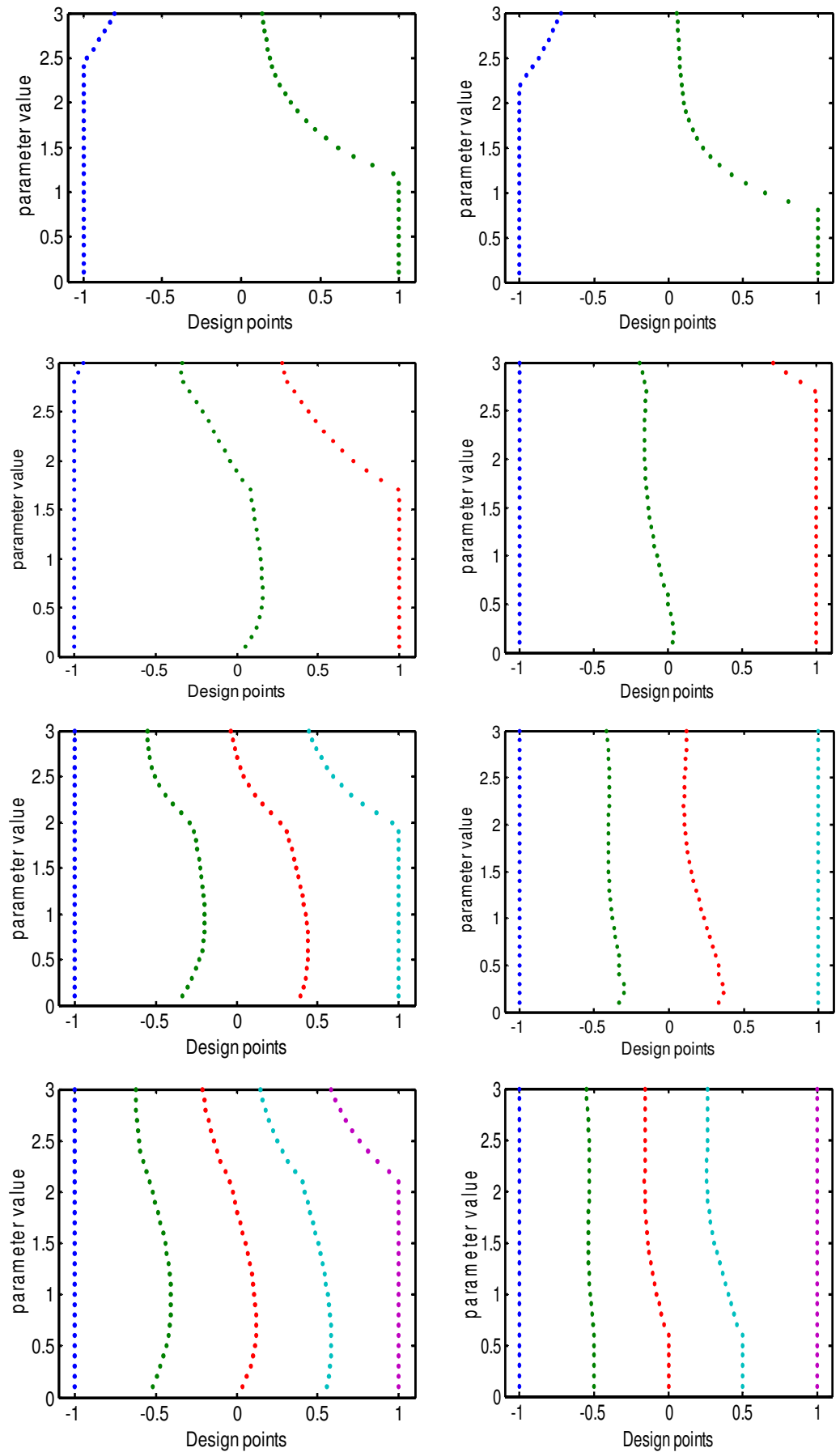

Figure 6. 2. Locally D-optimal allocations of time points for logistic mixed effects model.

When the number of time points $q$ increases, the optimal allocations of the additional time points are close to the optimal allocations of the $p$ time points for the PQL method, where $p$ is the number of regression parameters in the model (first column of Figure 6.2). This pattern is different when serial correlations are also included to the logistic mixed- 
effects model and the extended GEE method of analysis is considered. The optimal allocations of the additional time points move more away to the center from the optimal allocations of the $p$ time points for the extended GEE method in Figure 6.2 and the Doptimal allocations become more similar to equidistance designs.

\section{Maximin D-optimal designs}

Table 6.1 shows the results of the maximin D-optimal design with regression parameter values in $(0.0,3.0]$ and $(0.0,1.5)$. The MMEs are smaller when the range of the regression parameters is wider than when the range is narrow. For example, for a linear model with number of time points $q=2$ and the PQL method is used, the MME is 0.78 when the interval of the regression parameters is $(0.0,3]$ while the MME is 0.98 when the interval is $(0.0,1.5)$ (Table 6.1). In general, the MME is high enough $(>0.88)$ for parameter values in $(0.0,1.5)$. This implies that if a researcher has no information on the specific parameter values and uses the maximin D-optimal design; then, the loss in efficiency is at most $12 \%$. This loss in efficiency can be compensated by sampling (1/0.88-1)*100\% $(1 / 0.88-1) * 100 \% \approx 14 \%$ more subjects.

The smallest MME slightly decreases as the value of serial correlations increases. The MMEs also increase as the number of time points $q$ increases. However, this comparison is misleading because the two designs with different number of time points cannot be compared by the absolute measure MME. The relative efficiency should be used instead which takes into account the different numbers of time points. This comparison is given in the next section. 
Table 6.1. Maximin D-optimal designs for random effect models.

a) with regression parameter values in $(0.0,3]$

\begin{tabular}{|c|c|c|c|c|c|c|c|}
\hline model & $q$ & \multicolumn{2}{|l|}{ PQL } & \multicolumn{4}{|c|}{ Extended GEE method with AR(1) structure } \\
\hline & 2 & $-1.00,0.56$ & 0.78 & $-1.00,0.41$ & 0.76 & $-1.00,0.19$ & 0.76 \\
\hline \multirow[t]{2}{*}{ linear } & 4 & $-1.00,-0.90,0.52,0.62$ & 0.83 & $-1.00,-0.37,0.20,1.00$ & 0.98 & $-1.00,-0.42,0.12,1.00$ & 0.99 \\
\hline & 5 & $-1.00,-0.90,0.48,0.58,0.68$ & 0.83 & $-1.00,-0.52,-0.12,0.33,1.00$ & 0.99 & $-1.00,-0.55,-0.16,0.28,1.00$ & 0.99 \\
\hline quadratic & 3 & $-1.00,-0.19,0.76$ & $\mathbf{0 . 8 3}$ & $-1.00,-0.26,0.68$ & 0.83 & $-1.00,-0.28,0.55$ & 0.82 \\
\hline \multirow[t]{2}{*}{ cubic } & 4 & $-1.00,-0.57,0.15,0.80$ & 0.84 & $-1.00,-0.66,0.13,0.72$ & 0.85 & $-1.00,-0.65,0.18,0.64$ & 0.87 \\
\hline & 5 & $-1.00,-0.55,0.18,0.72,0.82$ & 0.86 & $-1.00,-0.65,0.03,0.58,1.00$ & 0.97 & $-0.99,-0.65,0.00,0.57,1.00$ & 0.94 \\
\hline
\end{tabular}

b) with regression parameter values in $(0.0,1.5)$.

\begin{tabular}{|c|c|c|c|c|c|c|c|}
\hline \multirow{3}{*}{ model } & \multirow[b]{3}{*}{$q$} & & & \multicolumn{4}{|c|}{ Extended GEE method with AR(1) structure } \\
\hline & & \multicolumn{2}{|l|}{ PQL } & \multicolumn{2}{|l|}{$\rho=0.5$} & \multicolumn{2}{|l|}{$\rho=0.9$} \\
\hline & & $\xi_{q}^{\text {maximin }}$ design & MME & $\xi_{q}^{\text {maximin }}$ design & MME & $\xi_{q}^{\operatorname{maximin}}$ design & MME \\
\hline & 2 & $-1.00,1.00$ & 0.98 & $-1.00,0.91$ & 0.95 & $-1.00,0.61$ & 0.89 \\
\hline & 3 & $-1.00,0.90,1.00$ & 0.99 & $-1.00,0.03,1.00$ & 0.99 & $-1.00,-0.13,1.00$ & 0.99 \\
\hline \multirow[t]{2}{*}{ linear } & 4 & $-1.00,0.80,0.90,1.00$ & 0.99 & $-1.00,-0.30,0.33,1.00$ & 0.99 & $-1.00,-0.41,0.17,1.00$ & 0.99 \\
\hline & 5 & $-1.00,-0.90,0.80,0.90,1.00$ & 0.99 & $-1.00,0.46,0.02,0.49,1.00$ & 0.99 & $-1.00,-0.54,-0.13,0.33,1.00$ & 0.99 \\
\hline \multirow{3}{*}{ quadratic } & 3 & $-1.00,-0.17,0.90$ & 0.92 & $-1.00,-0.29,0.82$ & 0.90 & $-1.00,-0.34,0.69$ & 0.88 \\
\hline & 4 & $-1.00,-0.14,0.82,0.92$ & 0.94 & $-1.00,-0.38,0.27,0.98$ & 0.96 & $-1.00,-0.41,0.43,1.00$ & 0.98 \\
\hline & 5 & $-1.00,-0.28,-0.18,0.84,0.94$ & 0.94 & $-1.00,-0.58,-0.15,0.44,1.00$ & 0.99 & $-1.00,-0.56,-0.10,0.51,1.00$ & 0.99 \\
\hline \multirow[t]{2}{*}{ cubic } & 4 & $-1.00,-0.55,0.17,0.88$ & 0.90 & $-1.00,-0.63,0.18,0.78$ & 0.89 & $-1.00,-0.64,0.14,0.71$ & 0.90 \\
\hline & 5 & $-1.00,-0.55,0.17,0.81,0.91$ & 0.92 & $-1.00,-0.66,0.07,0.60,1.00$ & 0.98 & $-1.00,-0.68,0.04,0.60,1.00$ & 0.98 \\
\hline
\end{tabular}




\section{Optimal number of time points}

Relative efficiencies of a maximin D-optimal design $\xi_{q}^{\operatorname{maximin}}$ compared to a maximin Doptimal design $\xi_{p}^{\text {maximin }}$ for the models considered in this chapter are computed to determine the most efficient number of time points over time. Figure 6.3 shows the relative efficiency plots with regression parameter values in $(0.0,1.5)$.

In Figure 6.3, the model with the number of parameters $p$ in bracket varies across the rows and the anticipated method of estimation varies across the columns. The plots for relative efficiencies of a maximin D-optimal design with $q$ number of time points compared to the maximin D-optimal design with $p$ number of time points based on PQL method are shown in the first column while the last two columns show the plots of relative efficiencies for logistic mixed-effects models with serial correlations (based on extended version of GEE method). In each plot, the vertical axis shows the relative efficiency (RE) and the horizontal axis shows the number of time points $q \geq p$.

From Figure 6.3, the relative efficiencies are below 1, which implies that, a maximin D-optimal design with number of time points $q=p$ is the most efficient design. This result is not surprising, as similar findings on optimal design have been reported in the literature for simple logistic models with independent observations (Silvey, 1980; Sebastiani and Settimi, 1997) and linear mixed-effects models (Tan and Berger, 1999 among others). Our numerical study shows that maximin D-optimal designs with the number of time points equal to the number of regression parameters in the model are the most efficient designs (Figure 6.3). 
model

PQL method

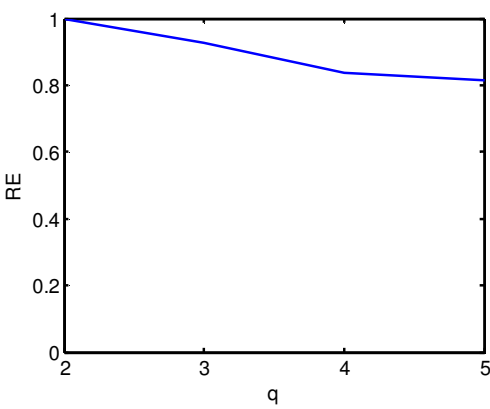

linear

$(p=2)$
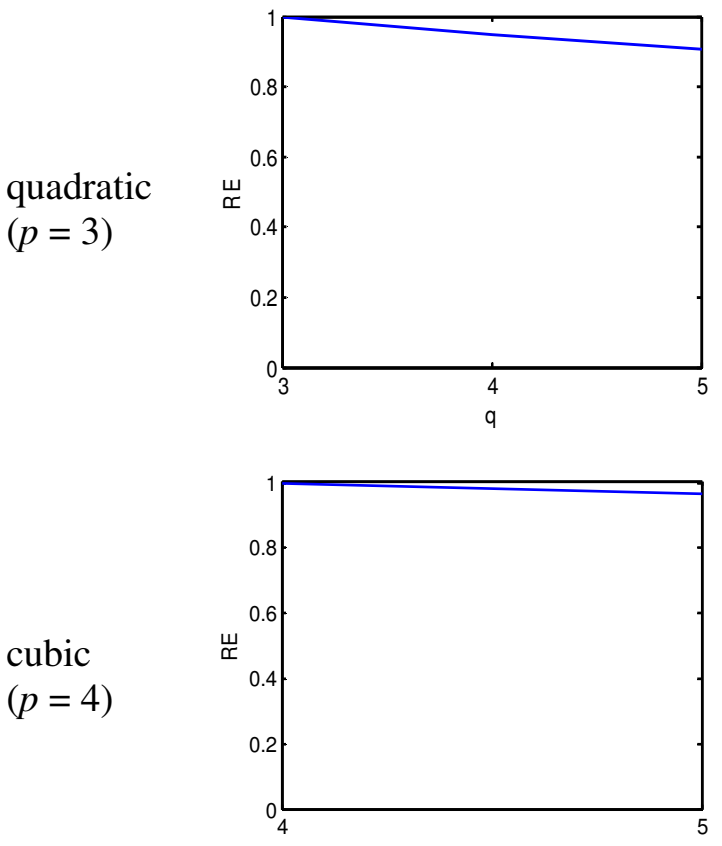

q
Extended GEE method with AR(1) structure

$\rho=0.5$
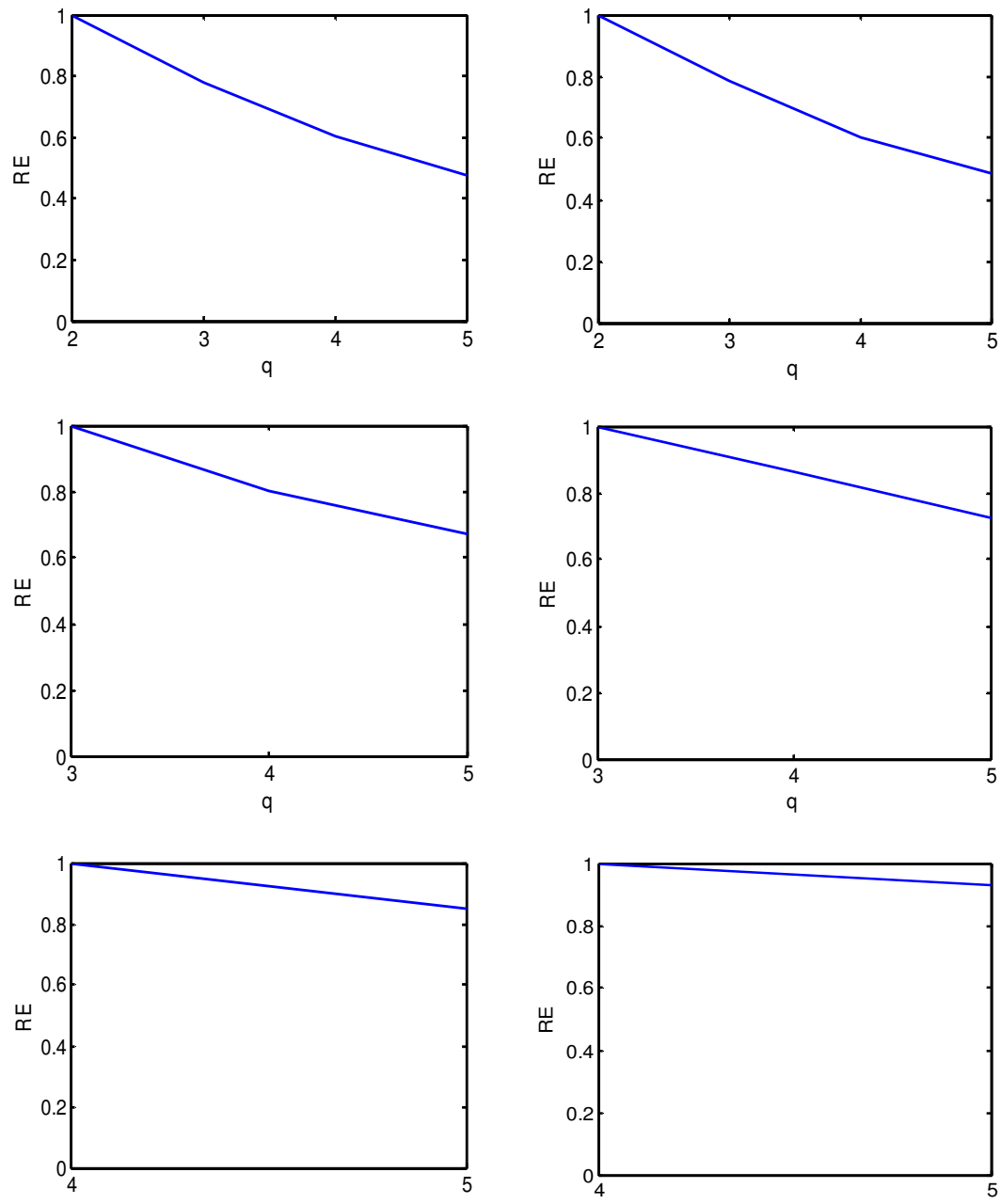

Figure 6.3. $\operatorname{RE}\left(\xi_{q}^{\operatorname{maximin}}, \xi_{p}^{\operatorname{maximin}}\right)$ for random effect models with regression parameter values in $(0.0,1.5)$.

As it is shown in Table 6.1, the last location of the design is shifted in most cases to the left from the end point of the time interval. However, in practice, study duration (the beginning and the end point of the time interval) is mainly based on logistical and practical feasibilities. Thus, a design has to contain the two end points of the study period. We have computed maximin optimal designs that include the two end points of the time interval. Table 6.2 presents these designs for the number of time points $q$ equal to the number of regression parameters $p$ in the model. These designs are denoted by $\xi_{p}^{\mathrm{EP}}$ as they include the End Points of the time interval. They are sub-optimal as compared to the corresponding maximin D-optimal designs and their MME are smaller compared to the MME of the maximin D-optimal designs. 
The MMEs for designs in Table 6.2 are high $(0.98,0.87$, and 0.79 for linear, quadratic and cubic models, respectively) when the there is no autocorrelation in the model (PQL method). The efficiencies are also high enough for moderate values of the autocorrelation $(\rho=0.5)$ and the degree of the polynomial is linear or quadratic. The MME is still relatively high for the linear model when the autocorrelation parameter is large.

Table 6.2. Maximin designs that include the end points of the time interval for random effect models with regression parameter values in $(0.0,1.5)$.

\begin{tabular}{|c|c|c|c|c|c|c|}
\hline \multirow{3}{*}{ model } & \multirow{2}{*}{\multicolumn{2}{|c|}{ PQL }} & \multicolumn{4}{|c|}{ Extended GEE method with AR(1) structure } \\
\hline & & & $\rho=0.5$ & & $\rho=0.9$ & \\
\hline & $\xi_{p}^{\mathrm{EP}}$ design & MME & $\xi_{p}^{\mathrm{EP}}$ design & MME & $\xi_{p}^{\mathrm{EP}}$ design & MME \\
\hline linear & $-1.00,100$ & 0.98 & $-1.00,1.00$ & 0.94 & $-1.00,1.00$ & 0.78 \\
\hline quadratic & $-1.00,-0.20,1.00$ & 0.87 & $-1.00,-0.30,1.00$ & 0.79 & $-1.00,-0.32,1.00$ & 0.69 \\
\hline cubic & $\begin{array}{c}-1.00,-0.56,0.16 \\
1.00\end{array}$ & 0.79 & $\begin{array}{c}-1.00,-0.67,0.11 \\
1.00\end{array}$ & 0.70 & $\begin{array}{c}-1.00,-0.67,0.12 \\
1.00\end{array}$ & 0.65 \\
\hline
\end{tabular}

\subsection{Example in practice}

The French EPIDOS study (Epidémiologie de l'ostéoporose) is a large multicentre, prospective study of the risk factors for hip fracture among elderly women, aged 75 years or older included in 1992-1993 (Dargent-Molina et al., 1996, Carrière and Bouyer, 2002). The evolution of disability based on data from Montpellier, one of the five participating centres, was analyzed by Carrière and Bouyer (2002). In their work on helpful strategies for choosing marginal and random effects models in longitudinal binary responses, they have concluded that a random effects model is the most suitable one for the analysis of self-reported disability in older women. It was reported that the binary response variable "needing help to go outdoors or home-confined" as an indicator of disability was collected annually for 6 years. We want to compare this 6-time point design with the proposed maximin designs.

The model considered by Carrière and Bouyer (2002) includes linear time effect. The square time effect was tested and found not significant. If a logistic mixed-effects model with linear predictor of time is considered to describe the evolution over time, then the maximin design for regression parameter values in an interval $(0,1.5)$ suggests taking measurements at the first year, and at the end of the follow-up period ( $6^{\text {th }}$ year). Equation (6.9) is used to compute the relative efficiency of the 6-time point design compared to the 2-time point maximin design and the $\mathrm{RE}=0.61$. This implies that the same variance for the 
regression parameter estimators could be obtained by sampling only about $61 \%$ of the subjects from the 6-time point design if the 2-time point maximin design were used. Unless it is known beforehand that a linear term of time is adequate, the higher degrees of time (time square and time cubic) have to be tested during the data analysis and the design should consider more design points. If the quadratic model is adopted, then the maximin design suggests taking measurements at the two end points and an additional third point. The additional time point should be considered at -0.20 in the $[-1,1]$ (see Table 6.2 for PQL method). By applying a linear transformation, the corresponding time point in the scale of the study period in years, $[1,6]$, is $6-5\left(\frac{1-(-0.20)}{2}\right)=3$. Thus, the maximin design suggests measuring in the first, third and sixth year. The RE of the 6-time point design compared to the 3-time point maximin design is 0.81 . Similarly, the 4-time point maximin designs points are at years 2, 4 and 6 for cubic trend of time with $\mathrm{RE}=0.95$ for the 6-point design compared to maximin design implying that $95 \%$ of the subjects are enough in 4-time point maximin design.

\subsection{Computer program}

We have developed a user-interface computer Program for Optimal design of Longitudinal Studies with Logistic models (POLS-Logistic) in MATLAB that helps the user to obtain optimal design results with a specification of the random effects models. The corresponding program for linear mixed-effects models is described in details in chapter 4 . POLS-Logistic helps to obtain locally D-optimal and maximin D-optimal designs. The optimization algorithm of POLS-Logistic uses mainly the fmincon function of MATLAB. The results described in previous sections are obtained using this program. We briefly describe the program in this section.

The program requires the user to specify input values on the variance-covariance parameters for random effects and range of parameter values. Then, the user obtains the optimal design results via the main menu of the program shown in Figure 6.4. 


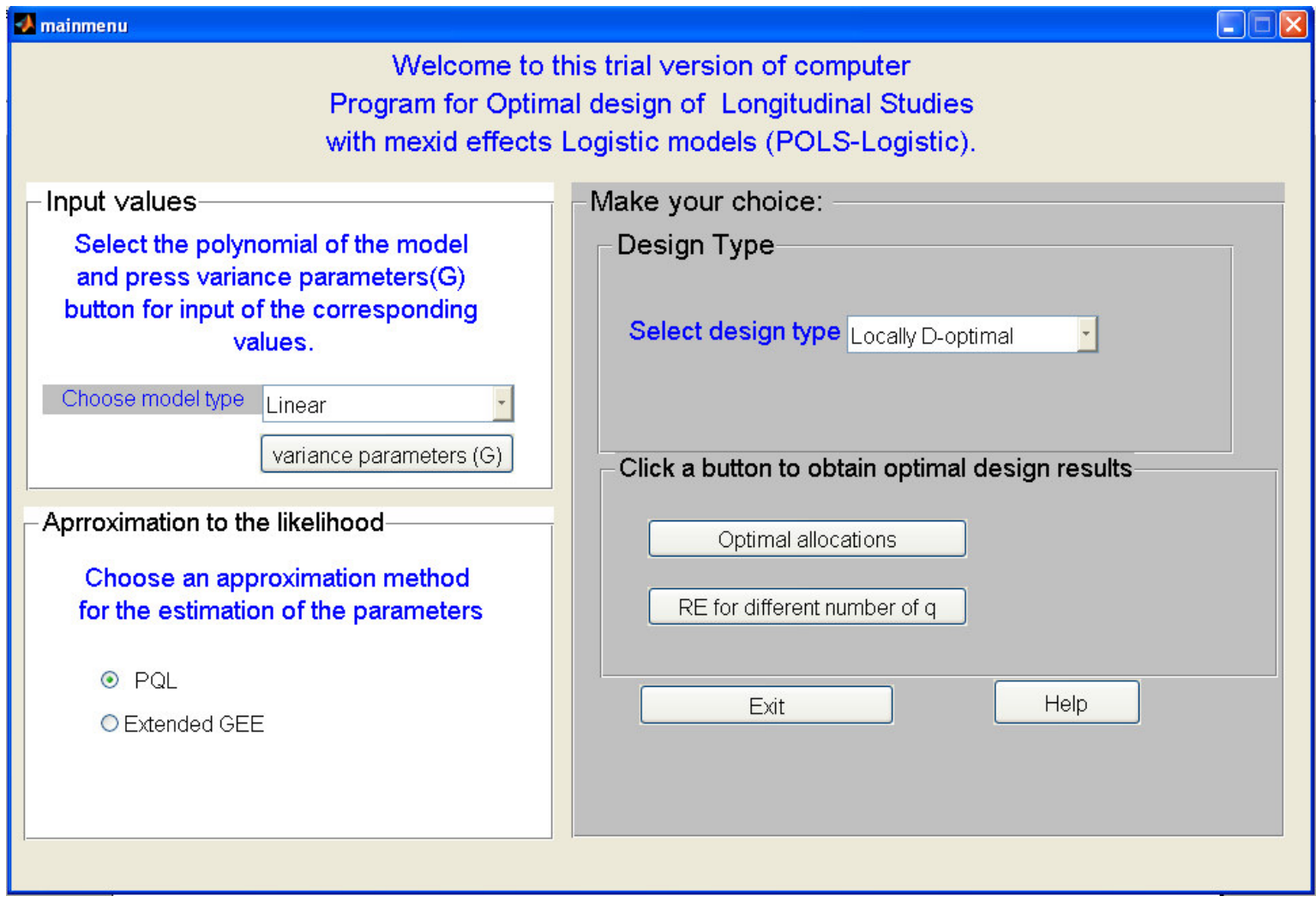

Figure 6.4. The main menu with the default input values for ODS4LOST-Logistic program.

\section{Using the software}

Typing mainmenu1 in MATLAB will initialize the program and show the main menu shown in Figure 6.4.

The user should give the necessary input parameters that are discussed below for the main menu.

\section{Input parameters}

- Choice of a polynomial: Linear, quadratic or cubic models. This choice can be based on knowledge from previous studies, a pilot study, biological/scientific expectations or expert opinion. The default polynomial of the mixed effects model is linear.

- Values of the variance-covariance parameters in $\boldsymbol{G}$ : The user will find a sub-menu for input of the values for the variance components in $\mathbf{G}$ matrix. If a fixed effects model is assumed, then all the values required in $\mathbf{G}$ should be set to zero.

- Approximation method: choose the approximation method for the estimation of the parameters. Input value of the serial correlation coefficient should also be given for the extended GEE method. 
- Type of design: making selection on locally D-optimal or maximin D-optimal designs. When the choice is maximin D-optimal design, a sub-menu for the input of range of parameter values will appear.

\section{Buttons/options to obtain output}

The following buttons are shown in Figure 6.4 to obtain results.

- 'Optimal allocations': the user will find dialog boxes to fill the specific number of time points. The optimal allocations within the time interval $[-1,1]$ for the specified number of time points will be computed. The relative efficiencies of this design and equidistance design compared to an optimal design with the number of time points $q=p$ will also be computed.

- 'RE for different number of $q$ ': this option gives the relative efficiency of designs with the number of time points $q>p$ compared to an optimal design with the number of time points $q=p$.

\section{Main output}

The allocations of the time points and the relative efficiencies are the main outputs of the program. The optimal allocations and relative efficiencies are displayed in figures as a function of the regression parameters (Figures 6.2 and 6.3). These results are displayed in an editable matlab figure and if required also in an excel table. Upon request, the program opens an excel file to print the results. The output of the program contains the optimal allocations and maximin efficiencies in the case of maximin D-optimal designs. The output also contains the relative efficiencies comparing efficiencies of

- optimal design for $q>p$ with optimal design for $q=p$,

- equidistance design for $q>p$ with optimal design for $q=p$.

A 'Help' button is also available in the main menu for guidance. The 'Exit' button in the main menu stops the program.

\subsection{Discussion and conclusions}

The problem of choosing an optimal design for logistic mixed-effects models for binary longitudinal responses is considered. Questions on the optimal number of repeated measurements and the locations of the measurements during the study period are 
addressed. Furthermore, a new interactive software program is introduced. The software can be obtained upon request from the authors.

The findings show that the optimal number of time points should not be too large as compared to the number of regression parameters $p$ in the model. Loss in efficiency increases as the number of time points increases. Similar findings have been reported in the literature for simple logistic models with independent observations (Silvey, 1980; Sebastiani and Settimi, 1997) and linear mixed-effects models (Tan and Berger, 1999 among others).

For logistic mixed-effects models the optimal allocations of the measurements depends on the values of the regression parameters. The optimal allocations include both edges of the time interval in most cases and are similar to the optimal allocations for the linear regression models. However, the optimal allocations for logistic mixed models move away from the edges of the time intervals and become different from that of the linear models as the parameter values increase (see Figure 6.2). When the parameter values are small, the curvature of the logit function is small and a straight line can approximate it. Thus, it is expected that the optimal designs for logistic mixed-effects models approximate those of the linear mixed regression models when the initial values of the regression parameters are small. When the parameter values increase, the curvature for the logit model also increases and it can no more be approximated by a straight line, implying that the optimal designs for a logistic model may not be similar to that of the linear model experiments. The results in this chapter are given only for positive values of the regression parameters. However, it must be noted that because of the symmetry of the logit link function, we will get similar designs for negative values of the regression parameters.

Maximin D-optimal designs are given to overcome the problem of parameter dependency of designs. The MME of these designs is high in most cases for parameter values in the interval $(0.0,1.5)$, implying that these designs are robust for the parameter values. The MME is relatively high especially when the autocorrelation parameter is not extremely large (Table 6.2).

Our recommendation is that, only designs with number of repeated measurements set close to the number of parameters should be chosen. Either determine the locations of the design points based on prior knowledge about the values of the regression parameters or consider the maximin design points. Values on specific regression parameters are often difficult to get at the design stage of a study. The maximin designs have high efficiency and they are robust over a range of parameter values. The locations of these design points 
shift to the left (Table 6.2) compared to the corresponding optimal design points for general linear mixed models (Tan and Berger, 1999).

Taking repeated measurements as close as the number of regression parameters instead of more repeated measurements not only helps to gain of efficiency but also eases the costs of data collection. The number of regression parameters in the model depends on degree of a polynomial that describes the evolution of events over time. In most cases a researcher may have a fairly good notion of the degree of a polynomial that will fit the data best. In case of doubt about the degree of the polynomial, whether it is linear, quadratic, or cubic model, an optimal design with 4 time points could be considered and the fit of the model can be checked. As is shown in Figure 6.3, the RE of this design compared to the optimal designs with $q=p$ time points is at least 0.80 in most cases.

This chapter focuses on the optimal designs for the estimation of parameters which describe the change over time within one group. The results could be extended for the purpose of comparing two or more groups over time. Although we expect the results not to be very different in these cases, further study will be needed to confirm this conjecture. It is also assumed that all subjects are available for the study over the study period and that there is no dropout. Further study on the effect of dropout on optimal designs is recommended for future study. 


\section{References}

Abdelbasit, and Plackett, R. L. (1983). Experimental Design for Binary Data. Journal of the American Statistical Association, 78, 90-98.

Abt, M., Gaffke, N., Liski, E.P., and Sinha, B.K. (1998). Optimal designs in growth curve models. Part II. Correlated models for quadratic growth: optimal designs for parameter estimation and growth prediction. Journal of statistical planning and Inference, 67, 287-296.

Abt, M., Liski, E.P., Mandal, N.K., and Sinha, B.K. (1997). Optimal designs in growth curve models. Part I. correlated models for linear growth: optimal designs for slope parameter estimation and growth prediction. Journal of statistical planning and Inference, 64, 141-150.

Astin, J.A. (1981). Why patients use alternative medicine. Results of a national study. Journal of the American Medical Association, 279, 1548-53.

Atkins, J.E, and Cheng, C.S. (1999). Optimal regression designs in the presence of random block effects. Journal of Statistical Planning and Inference, 77, 321-335.

Atkinson, A.C. and Donev, A.N. (1996). Optimal Experimental Designs. Oxford: Clarendon press.

Atkinson, A.C., Chaloner, K., Herzberg, A.M., and Juritz, J., (1993). Optimum experimental designs for properties of a compartmental model. Biometrics, 49, 325337.

Bandera, E.V., Freudenheim, J.L., Marshall, J.R., Zielenzy, M., Priore, R.L., Brasure, J., Baptiste, M., Graham, S. (1997). Diet and alcohol consumption and lung cancer risk in the New York state Cohort (United States). Cancer Causes and Control, 8, 828840.

Berger, M.P.F. (1986). A comparison of efficiencies of longitudinal, mixed longitudinal and cross-sectional designs. Journal of Educational Statistics, 11, 171-181.

Bischoff, W. (1993). On D-optimal designs for linear models under correlated observations with an application to a linear model with multiple response. Journal of Statistical Planning and Inference, 37, 69-80.

Braess, D., and Dette, H. (2007). On the Number of support points of Maximin and Bayesian Optimal designs. The Annals of Statistics, 35, 772-792.

Breslow, N.E., and Clayton, D.G. (1993). Approximate Inference in Generalized Linear Mixed Models. Journal of the American Statistical Association, 88, 9-25. 
Bruijn, G.J., Kremers, S.P.J., Mechelen, W.V., Brug, J. (2005). Is personality related to fruit and vegetable intake and physical activity in adolescents? Health Education Research, 20, 635-644.

Calle, E.E., Rodriguez, C., Jacobs, E.J., Almon, M.L., Chao, A., McCullough, M.L., Feigelson, H.S., and Thun, M.J. (2002). The American Cancer Society Cancer Prevention Study II Nutrition Cohort. Cancer, 94, 500-511.

Camargo, C.A., and Willett, W.C. (1999). Prospective Study of Body Mass Index, Weight Change, and Risk of Adult-onset Asthma in Women. Archives of Internal Medicine, $159,2582-2588$.

Carr, A., Chuah, J., Hudson, J., French, M., Hoy, J., Law, M., Sayer, D., Emery, S., and Cooper, D.A. (2000). A randomized, open-label comparison of three highly active antiretroviral therapy regimens including two nucleoside analogues and indinavir for previously untreated HIV-1 infection: the OzCombo1 study. AIDS, 14, 1171-1180.

Carrière, I., and Bouyer, J. (2002). Choosing marginal or random effects models for longitudinal binary responses: application to self-reported disability among older persons. BMC Med Res Methodology, 2:15. doi: 10.1186/1471-2288-2-15.

Chaloner, K., (1989). Bayesian design for estimating the turning point of a quadratic regression. Communications in Statistics-Theory Methods, 18, 1385-1400.

Chaloner, K., and Larntz, K., (1989). Optimal Bayesian design applied to logistic regression experiments. Journal of Statistical Planning and Inference, 21, 191-208.

Chaloner, K., and Verdinelli, I., (1995). Bayesian experimental design: a review. Statistical Science, 10, 273-304.

Chang, F-C., and Lay, C-F. (2002). Optimal designs for growth curve models. Journal of statistical planning and Inference, 104, 427-438.

Chernoff, H. (1953). Locally optimal designs for estimating parameters. Annals of Mathematical Statistics, 24, 586-602.

Chipman, H. and Welch, W. (1996). D-optimal Designs for Generalized Linear Models, unpublished manuscript downloaded from http://citeseer.ist.psu.edu/144155.html.

Cnaan, A, Laird, N.M., and Slasor, P. (1997). Tutorial in Biostatistics: Using the general linear mixed model to analyse unbalanced repeated measures and longitudinal data. Statistics in Medicine, 16, 2349-2380.

Colditz, G.A. (1994). Oral Contraceptive use and Mortality during 12 years of Follow-up: The Nurses' Health Study. Annals of Internal Medicine, 120, 821-826.

Dargent-Molina, P., Faver, F., Grandjean, H., Baudoin, C., Schott, A.M., Hausherr, E., et 
al. (1996). Fall-related factors and risk of hip fracture: the EPIDOS prospective study. Lancet, 348, 145-49.

DasGupta, A., (1996). Review of optimal Bayes designs. In: Ghosh, S., Rao, C.R. (Eds.), Handbook of Statistics, Vol. 13. Amsterdam: Elsevier, pp. 1099-1148.

Dawber, T.R. (1980). The Framingham study: the epidemiology of atherosclerotic disease. Harvard : Harvard University Press.

Deeks, S.G., Hecht, F.M., Swanson, M., Elbeik, T., Loftus, R., Cohen, P.T., and Grant, R.M. (1999). HIV RNA and CD4 cell count response to protease inhibitor therapy in an urban AIDS clinic: response to both initial and salvage therapy. AIDS, 13, 35-43.

Dette, H. (1996). A Note on Bayesian C-and D-optimal designs in nonlinear regression models. The Annals of Statistics, 24, 1225-1234.

Dette, H., (1997). Designing of experiments with respect to "standardized" optimality criteria. Journal of Royal Statistical Society B, 59, 97-110.

Dette, H., and Sahm, M., (1997). Standardized optimal designs for Binary response experiments. South African Statistical Journal, 31, 271-298.

Dette, H., and Sperlich, S. (1994). A note on Bayesian D-optimal designs for a generalization of the exponential growth model. South African Statistical Journal, 28, 103-117.

Diehr, P., Martin, D.C., Koepsell, T., Cheadle, A., Wagner, E., and Psaty, B. (1995). Optimal survey design for community intervention evaluations: cohort or crosssectional? Journal of Clinical Epidemiology, 48, 1461-72.

Diggle, P.J. (1988). An approach to the analysis of repeated measures. Biometrics, 44, 959971.

Diggle, P.J., Liang, K.-Y., and Zeger, S.L. (1994). Analysis of Longitudinal Data. Oxford: Oxford University Press.

Dik, M.G., Deeg, D.J.H., Bouter, L.M., Corder, E.H., Kok, A., Jonker, C. (2000). Stroke and Apolipoprotein E c4 Are Independent Risk Factors for Cognitive Decline: A population-Based Study. Stroke, 31, 2431-2436.

Fedorov, V.V. (1972). Theory of Optimal Experiments. New York: Academic Press.

Fedorov, V.V., and Müller, W.C., (1997). Another view on optimal design for estimating the point of extremum in quadratic regression. Metrika, 46, 147-157.

Fedorov, V.V., Gagnon, R.C., Leonov, S.L. (2002). Design of experiments with unknown parameters in variance. Applied Stochastic Models in Business and Industry, 18, 207218. 
Fletcher, R. (1987). Practical Methods of Optimization. 2nd ed. Chichester: John Wiley \& Sons.

Gagnon, R., and Leonov, S. (2005). Optimal Population Designs for PK models with serial Sampling. Journal of Biopharmaceutical Statistics, 15, 143-163.

Goldstein, H. (1979). The Design and Analysis of Longitudinal Studies. London: Academic press.

Han, C., and Chaloner, K. 2004. Bayesian Experimental Design for Nonlinear MixedEffects Models with Application to HIV Dynamics. Biostatistics, 60, 25-33.

Hand, D., and Crowder, M. (1996). Practical Longitudinal Data analysis. London: Chapman and Hall.

Imhof, L.A., (2001). Maximin designs for exponential growth models and heteroscedastic polynomial models. Annals of Statistics, 29, 561-576.

Khuri, A. I., Mukherjee, B., Sinha, B. K., and Ghosh, M. (2006). Design Issues for Generalized Linear Models: A review. Statistical Science, 21, 376-399.

King, J., and Wong, W.K. (2000). Minimax D-optimal designs for the logistic model. Biometrics, 56, 1263-1267.

Laird, N. M., and Ware, J. H., (1982). Random-effects models for longitudinal data. Biometrics, 38, 963-974.

Liang, K.-Y., and Zeger, S.L. (1986). Longitudinal Data Analysis using Generalized Linear Models. Biometrika, 73, 13-22.

Lindsey, J.K. (1993). Models for Repeated Measurements. Oxford: Clarendon.

Machin, D. (1975). On a design problem in growth studies. Biometrics, 31, 749-753.

Mathew, T., and Sinha, B.K. (2001). Optimal designs for binary data under logistic regression. Journal of Statistical Planning and Inference, 93, 295-307.

Matlab (2004). Handbook MATLAB. Version 7.0.1. (R14), MathWorks Inc.

McCullagh, P., and Nelder, J.A. (1989). Generalized Linear Models. London: Chapman \& Hall.

McCulloch, C. E., and Searle, S. R., (2001). Generalized, Linear and Mixed Models. New York: Wiley.

Mentre, F., Mallet, A., and Baccar, D. (1997). Optimal design in random effect regression models. Biometrika, 84, 429-442.

Moerbeek, M. (2005). Robustness properties of A-, D-, and E-optimal designs for polynomial growth models with autocorrelated errors. Computational statistics \& data analysis, 48, 765-778. 
Molenberghs, G., and Verbeke, G. (2005). Models for Discrete Longitudinal Data. New York: Springer.

Moroldo, M.B., Chaudhari, M., Shear, E., Thompson, S.D., Glass, D.N, and Giannini, E.H. (2004). Juvenile rheumatoid arthritis affected sibpairs. Extent of clinical phenotype concordance. Arthritis \& Rheumatism, 50, 1928-1938.

Mukhopadhyay, S. and Haines, L.M. (1995). Bayesian D-optimal designs for the exponential growth model. Journal of Statistical Planning and Inference, 44, 385397.

Müller, C. and Pazman, A. (1998). Applications of necessary and sufficient conditions for maximin efficient designs. Metrika, 48, 1-19.

Müller, Ch.H., (1995). Maximin efficient designs for estimating nonlinear aspects in linear models. Journal of Statistical Planning and Inference, 44, 117-132.

Ortega-Azurdy, S.A., Tan, F.E.S., and Berger, M.P.F. (2007). The effect of dropout on the efficiency of D-optimal designs of linear mixed models. Statistics in Medicine, early view, DOI: 10.1002/sim.3108 (in press).

Ouwens, M.J.N.M, Tan, F.E.S., and Berger, M.P.F. (2002). Maximin D-optimal Designs for Longitudinal Mixed Effects Models. Biometrics, 58, 735-741.

Ouwens, M.J.N.M., Tan, F.E.S., and Berger, M.P.F. (2006). A maximin criterion for the logistic random intercept model with covariates. Journal of Statistical Planning and Inference, 136, 962-981.

Pinheiro, J.C., and Bates, D.M. (2000). Mixed Effects Models in S and S-Plus. New York: Springer-Verlag.

Pocock, S.J. (1983). Clinical trials: A practical approach. Chichester: John Wiley \& Sons.

Potthof, R.F., and Roy, S.N. (1964). A generalized multivariate analysis of variance model useful especially for growth curve problems. Biometrika, 51, 313-326.

Pukelsheim, F. (1993). Optimal design of experiments. New York: John Wiley \& Sons.

Rao, C,R. (1973). Linear statistical Inference and Its Applications. New York: John Wiley \& Sons.

Rao, M.N., and Rao, C.R. (1966). Linked cross-sectional study for determining norms and growth rates-A pilot survey on Indian school-going boys. Sankhy, Indian Journal of statistics series B, 28, 237-258.

Sebastiani, P., and Settimi, R. (1997). A note on D-optimal designs for a logistic regression model. Journal of Statistical Planning and Inference, 59, 359-368.

Silvey, S. D. (1980). Optimal Design. New York: Chapman and Hall. 
Sitter, R.R., and Wu, C.F.J. (1999). Two-stage design of quantal response studies. Biometrics, 55, 396-402.

Sitter, R. R., and Wu, C.F.J. (1993). Optimal designs for binary response experiments: Fieller-, D-, and A- criteria. Scandinavian Journal of Statistics, 20, 329-342.

Sitter, R.R., and Forbes, B.E. (1997). Optimal Two-Stage Designs for Binary Response Experiments. Statistica Sinica, 7, 941-955.

Skrondal, A., and Rabe-Hesketh, S. (2004). Generalized Latent Variable Modeling: Multilevel, Longitudinal, and Structural Equation Models. Boca Raton, FL: Chapman \& Hall/ CRC.

Stiratelli, R., Laird, N.M., and Ware, J.H. (1984). Random effects Models for Serial Observations with Binary Responses. Biometrics, 40, 961-971.

Tan, F.E.S., and Berger, M.P.F. (1999). Optimal allocation of time points for the random effects model. Communication in Statistics-Simulation and computation, 28, 517540 .

Tekle, F.B, Tan, F.E.S., and Berger, M.P.F. (2007). D-optimal cohort designs for linear mixed effects models. Statistics in Medicine, early view, DOI: 10.1002/sim.3045.

Tekle, F.B., Tan, F.E.S., and Berger, M.P.F. (2008). Too many repeated measurements is a waste of resources. Submitted.

Van den Akker, M., Spigt, M.G., De Raeve, L., Van Steenkiste, B., Metsemakers, J.F.M., Van Voorst, E.J., De Vries, H., (2008). The SMILE study: A study of Medical Information and Lifestyles in Eindhoven. The rationale and contents of a large prospective dynamic cohort study. BMC Public health, 8(19).

Van den Brandt, P.A., and Bausch-Goldbohm, S. (1993). A prospective cohort study on diet and cancer in the Netherlands: design, conduct, analysis and first results after 3.3 years of follow-up. Doctoral thesis, Universitaire Pers Maastricht, Maastricht

Verbeke, G., and Molenberghs, G. (2000). Linear Mixed Models for Longitudinal Data. New York: Springer.

Winkens, B., Schouten, H. J.A., van Breukelen, G. J. P., and Berger, M. P. F. (2007). Optimal designs for clinical trials with second-order polynomial treatment effects. Statistical Methods in Medical Research, 16, 523 - 537.

Winkens, B., Schouten, H. J.A., van Breukelen, G. J. P., and Berger, M. P. F. (2006). Optimal number of repeated measures and group sizes in clinical trials with linearly divergent treatment effects. Contemporary Clinical Trials, 27, 57 - 69.

Winkens, B., Schouten, H. J.A., van Breukelen, G. J. P., and Berger, M. P. F. (2005). 
Optimal time-points in clinical trials with linearly divergent treatment effects. Statistics in Medicine, 24, 3743 - 3756.

Woolson, R.F., Leeper, J.D., and Clark, W.R. (1978). Analysis of incomplete data from longitudinal and mixed longitudinal studies. Journal of the Royal Statistical Society, $141,242-252$.

Wu, C.F.J., (1985). Efficient sequential designs with binary data. Journal of American statistical Association, 80, 974-984.

Zacks, S., (1977). Problems and approaches in design of experiments for estimation and testing in non-linear models. In: P.R. Krishnaiah, Editor, Multivariate Analysis vol. 4, North-Holland, Amsterdam, pp. 209-223.

Zeger, S. L. and Liang, K.-Y., (1992). An overview of methods for the analysis of longitudinal data. Statistics in Medicine, 11, 1825-1839.

Zeger, S.L., Liang, K.-Y., and Albert, P.S. (1988). Models for Longitudinal Data: A Generalized Estimating Equation Approach. Biometrics, 44, 1049-1060.

Zhu, W., Ahn, H., and Wong, W.K. (1998). Multiple-Objective Optimal Designs for the Logit model. Communications in Statistics- Theory and Methods, 27, 1581-1592. 

7 Summary and conclusion 


\subsection{Summary and conclusion}

Generalized Linear Mixed Models (GLMM) have been used quite effectively to model longitudinal data with discrete as well as continuous outcome variable. The choice of a design for GLMM is a very important task in the development and building of an adequate model. In addition to an appropriate method of analysis, a carefully designed study will safeguard the validity of the final conclusion of longitudinal studies. Optimal planning of research designs may also save money and time by specifying the optimal number of cohorts, time points, and optimal allocation of measurement points per subject with limited cost.

The objectives of this thesis are:

- to find the optimal number of cohorts,

- to identify the optimal number of repeated measurements per subject, and

- to determine the optimal allocations of time points within a study period for longitudinal studies with the constraint of the study cost (budget).

Chapter 1 is introductory. The GLMM and its two specific models namely the linear and logistic mixed models for continuous and dichotomous outcome variables respectively are described in this chapter. Designs for linear models are studied in chapters 2, 3 and 4 while designs for logistic models are studied in chapters 5 and 6. The D-optimality criterion is used in this thesis and is discussed in chapter 1. This criterion is popular because of its interpretation in terms of the volume of a confidence ellipsoid of the parameter estimators and its nice property that the D-optimal designs do not depend on the chosen time interval. The cost parameters encountered in longitudinal studies are introduced and discussed in the first chapter and implemented in optimization of designs in chapters 2,3 and 4 . The relative efficiency that helps to compare different designs is also discussed in the first chapter.

In chapter 2, we studied and identified optimal number of time points and allocations for longitudinal studies with fixed costs. Linear mixed models with polynomials of degree 1, 2 and 3 are considered. A numerical study shows that the optimal design results for a linear mixed model with cost constraints are almost similar to the results in literature for fixed effects linear models. It is found that the optimal number of time points is equal to the number of regression parameters in the mixed model except when the ratio of costs of recruiting a subject to the costs of additional measurement per subject is larger than about 20 and the autocorrelation coefficient is close to 0 . The optimal allocations are almost at 
equi-distance time points except when the autocorrelation coefficient between successive measurements within a subject is less than 0.1. In most practical cases, correlations between successive measurements per subject in longitudinal studies are expected to be greater or equal to 0.1 . Hence, in most practical cases, the optimal number of time points per subject is equal to the number of regression parameters and the optimal allocations are approximately equi-distant. From the numerical study, the main factors that affect the optimal design are the number of regression parameters in the model, the ratio between the cost of recruiting a new subject and the cost of an additional observation per subject, and the autocorrelation coefficient between repeated measurements per subject.

Depending on the number of cohorts (groups) over time, the design for longitudinal studies may be a purely longitudinal design, with only one cohort measured over the complete time interval, a mixed longitudinal design, with several cohorts measured over a shorter time interval for each cohort and a cross-sectional design, where groups of subjects are measured at a single time point for each group (Rao and Rao, 1966; Woolson et al., 1978). The purely longitudinal cohort design is studied in Chapter 2.

In chapter 3, the extension to other cohort designs is made by considering more than one cohort of subjects over time, i.e. purely longitudinal, mixed longitudinal and crosssectional cohort designs are considered in chapter 3 . The general finding shows that the optimal number of time points is a function of the number of cohorts and the number of regression parameters in the model. It is found that the optimal number of time points is the sum of the number of cohorts and the number of regression parameters in the model minus 1. Comparison of the three cohort designs (Purely longitudinal, mixed longitudinal and cross-sectional cohort designs) shows that the purely longitudinal cohort design with one cohort of subjects over the study period is the most efficient design. Thus, unless there is some biological or practical reason to consider, it is recommended to follow one group of subjects over the study period and take their measurements at the optimal allocation points which in most cases are approximately the same as the equi-distance time points.

In chapter 4, an interactive user-friendly computer program is introduced and described. This computer program is written in matlab to implement the basic ideas discussed in chapters 2 and 3. The program helps practitioners to obtain optimal cohort design with an optimal number of repeated measurements and optimal allocations of time points for longitudinal studies without going in to the details of optimal design theory. It also helps to computes the loss in efficiency of any alternative design compared to the optimal design. 
In chapter 5, designs for fixed effects logistic models with categorical independent variables are considered. It is known that designs for non linear models including logistic models depend on the model parameters that are not known before data collection. We proposed the use of what we call r-design in chapter 5 to go around this problem. The rdesign assigns an equal weight to design points that give more information in estimating the regression parameters and ignores those design points which contain less information. This design is D-optimal on a restricted design region. The $r$ - design can be constructed in practice by using the approximate rank order of the response variances at the design points. The design points are finite when all the independent variables are categorical. A numerical study shows that the r-designs are highly efficient in most cases as compared to the usual balanced designs that assign equal weight to all design points.

In chapter 6, designs for logistic mixed effects models for binary longitudinal data are considered. Locally D-optimal designs are studied and they are given as a function of the regression parameters. Maximin D-optimal designs are considered to solve the problem of parameter dependency of designs for logistic mixed models. Maximin D-optimal designs at a given interval of parameter values are computed by means of a maximin relative efficiency measure. The performance of the maximin D-optimal designs in terms of the maximin efficiency is high for a range of parameter values that is common in practice. Further, maximin designs with different numbers of time points are compared. The results

show that the optimal number of repeated measurements depends on the number of regression parameters in the model. The design locations generally shift to the left as compared to the design locations for linear mixed effects models known in the literature. A user-friendly computer program that helps to obtain locally and maximin D-optimal designs for logistic mixed models is also described in chapter 6.

\subsection{Final Remarks}

The most commonly used D-optimality criterion which is based on minimization of the volume of a confidence ellipsoid is considered in this thesis. Even if the volume of the confidence ellipsoid is minimized, a long thin ellipsoid orientated along or close to the parameter axes could also result in comparatively large variances of one or more parameters. Thus, it is also important to carefully study the lengths of the ellipsoid of the parameter estimators. The E- criterion minimizes the square of the length of the largest 
axis of the ellipsoid while the A-criterion minimizes the sum of the squares of the lengths of all axes. We recommend a future study that investigates E- and A-optimal designs for longitudinal studies and compares the findings with results in the current thesis.

The D-optimality is used to estimate all the parameters in a model as precisely as possible. If interest is in estimating a subset of the parameters in the model as precisely as possible, $D_{s}$-optimality criterion can be used. $D_{\mathrm{s}}$-optimality criterion minimizes the determinant of the variance-covariance submatrix of the estimators of $s$ chosen model parameters (Silvey, 1980; Atkinson and Donev, 1996). An investigation on $\mathrm{D}_{\mathrm{s}}$-optimality criterion for longitudinal studies is a topic of interest for the future.

It is assumed in this thesis that recruited subjects will be available during the whole study period, i.e., complete data are assumed. Drop-out is common in practice and it is not known yet how such drop-out will influence the optimality of suggested in this thesis. Ortega-Azurdy et al. (2007) concluded that if one uses a D-optimal design based on complete data and dropout is encountered during data collection, the loss of efficiency does not exceed $15 \%$. Thus, one can compensate for the small loss by increasing the initial sample size by at most $15 \%$. However, further research is needed for more general conclusions on the effect of drop-out on the optimality of a longitudinal design.

Covariates other than the polynomials over time are not incorporated in the evaluated models. Models with more covariates are often encountered in applied areas. However, the effect of inclusion of more covariates is yet to be studied.

Chapter 5 is restricted to categorical independent variables. Because continuous or a mix of continuous and categorical independent variables for logistic model are often encountered in practice, chapter 5 may further be extended to continuous and/or a mix of categorical and continuous independent variables. 


\section{Dutch Summary (Samenvatting)}

De Generalized Linear Mixed Models (GLMM) worden op een effectieve wijze gebruikt om longitudinale data met discrete evenals continue uitkomstvariabelen te modelleren. De keuze van een design voor GLMM is zeer belangrijk in de ontwikkeling van een geschikt model. Naast een aangewezen methode van analyse, zal een zorgvuldig gekozen design (proefopzet) de geldigheid van de resultaten van longitudinale studies beschermen. De optimale planning van onderzoeksdesigns kan ook geld en tijd besparen, door het optimale aantal cohorten, tijdstippen en een optimale toewijzing van meetmomenten per individu met beperkte kosten te specificeren.

De doelstellingen van dit proefschrift zijn de bepaling van:

- het optimale aantal cohorten,

- het aantal herhaalde metingen per individu, en

- de optimale toewijzing van tijdstippen binnen een studieperiode voor longitudinale studies, rekening houdend met de totale kosten van de studie (budget).

Hoofdstuk 1 heeft een inleidend karakter. GLMM en twee specifieke modellen worden in dit hoofdstuk beschreven, namelijk de lineaire en logistische mixed models voor respectievelijk continue en dichotome uitkomstvariabelen. De designs voor lineaire modellen komen in hoofdstukken 2,.3 en 4 aan de orde, terwijl de designs voor logistische modellen in hoofdstukken 5 en 6 worden besproken. Het D-Optimaliteitscriterium wordt in dit proefschrift gebruikt en besproken in hoofdstuk 1. Dit criterium is populair vanwege haar interpretatie in termen van het volume van een betrouwbaarheidsellipsoïde van de parameterschatters en het feit dat de D-Optimale designs niet afhankelijk zijn van het gekozen tijdinterval. De kostenparameters die in longitudinale studies veelal worden aangetroffen, worden hier geïntroduceerd en in het eerste hoofdstuk besproken en vervolgens geïmplementeerd bij het optimaliseren van designs in hoofdstukken 2, 3 en 4 . De relatieve efficiency om verschillende designs te vergelijken wordt ook in het eerste hoofdstuk besproken.

In hoofdstuk 2 bepaalden wij het optimale aantal tijdstippen en toewijzingen voor longitudinale studies met vooropgestelde kosten. De lineaire mixed models met polynomen van graad 1, 2 en 3 kwamen aan de orde. Een numerieke studie toont aan dat de optimale designresultaten voor een lineair mixed model met kostenbeperkingen vrijwel identiek zijn aan de resultaten in de literatuur voor fixed-effects lineaire modellen. Het optimale aantal tijdstippen blijkt gelijk te zijn aan het aantal regressieparameters in het mixed model, 
behalve wanneer de kosten om een individu te rekruteren ten opzichte van de kosten van een extra meting per individu groter is dan ongeveer 20 en de autocorrelatiecoëfficiënt bijna 0 is. De equidistante tijdstippen blijken nagenoeg optimaal te zijn, behalve wanneer de autocorrelatiecoëfficiënt tussen opeenvolgende metingen binnen een individu minder is dan 0,1 . In de meeste praktische gevallen verwacht men dat de correlaties tussen opeenvolgende metingen bij een individu in longitudinale studies groter of gelijk zijn aan 0,1 . Daarom is het optimale aantal tijdstippen per individu in de meeste praktische gevallen gelijk aan het aantal regressieparameters en de optimale toewijzingen zijn ongeveer equidistant. Uit de numerieke studie blijkt dat er een aantal belangrijke factoren zijn die het optimale design beïnvloeden, nl. het aantal regressieparameters in het model, de verhouding tussen de kosten om een nieuw individu te rekruteren en de kosten van een extra observatie per individu, en de autocorrelatiecoëfficiënt tussen herhaalde metingen per individu.

Afhankelijk van het aantal cohorten (groepen) over de tijd, kan het design voor longitudinale studies een puur longitudinaal design zijn met slechts één cohort die over het volledige tijdinterval wordt gemeten, een mixed longitudinaal design met verscheidene cohorten die over een korter tijdinterval worden gemeten en een cross-sectioneel design waar de groepen op één enkel tijdpunt voor elke groep worden gemeten (Rao en Rao, 1966; Woolson et al, 1978). Het puur longitudinaal cohortdesign wordt in hoofdstuk 2 bestudeerd.

In hoofdstuk 3 wordt de uitbreiding naar andere cohort-designs gemaakt door meer dan één cohort van individuen over tijd te overwegen, d.w.z. de puur longitudinale, de mixed longitudinale en de cross-sectionele cohort-designs worden in hoofdstuk 3 beschouwd. De algemene bevindingen tonen aan dat het optimale aantal tijdstippen een functie van het aantal cohorten en het aantal regressieparameters in het model is. Het blijkt dat het optimale aantal tijdstippen de som is van het aantal cohorten en het aantal regressieparameters in het model minus 1 . Een vergelijking van de drie cohort-designs (de puur longitudinale, de mixed longitudinale en de cross-sectionele cohort-designs) toont aan dat het puur longitudinaal cohort-design met één cohort van individuen tijdens de studieperiode het meest efficiënte design is. Tenzij er een biologische of praktische reden is waar men rekening mee dient te houden, wordt dus geadviseerd om één groep individuen tijdens de studieperiode te volgen en hun metingen op de optimale toewijzingspunten op te nemen die in de meeste gevallen ongeveer dezelfde zijn als de equidistante tijdstippen. 
In hoofdstuk 4 wordt een interactief gebruikersvriendelijk computerprogramma voorgesteld en beschreven. Dit computerprogramma werd geschreven in Matlab en implementeert de basisideeën die in hoofdstukken 2 en 3 werden besproken. Het programma helpt mensen uit de praktijk om een optimaal cohort-design met een optimaal aantal herhaalde metingen en optimale toewijzingen van tijdstippen voor longitudinale studies te verkrijgen zonder in detail te gaan over optimale design theorie. Het helpt ook het verlies in efficiency te berekenen van een alternatief design ten opzichte van het optimale design.

In hoofdstuk 5 worden de designs voor fixed-effects logistische modellen met categorische onafhankelijke variabelen beschouwd. Het is bekend dat de designs voor nietlineaire modellen inclusief logistische modellen afhankelijk zijn van de modelparameters die meestal onbekend zijn vóór de gegevensverzameling. In hoofdstuk 5 stelden wij voor om een $r$-design te gebruiken om dit probleem te omzeilen. Het $r$-design wijst een gelijk gewicht toe aan designpunten welke meer informatie geven bij het schatten van de regressieparameters en negeert die designpunten die minder informatie bevatten. Dit design is D-optimaal in een beperkt designgebied. Het $r$-design kan in de praktijk worden geconstrueerd door de benaderende rangorde van de varianties van de uitkomstvariabele op de designpunten te gebruiken. De designpunten zijn begrensd wanneer alle onafhankelijke variabelen categorisch zijn. Een numerieke studie toont aan dat de $r$-designs in de meeste gevallen zeer efficiënt zijn in vergelijking met de gebruikelijke gebalanceerde designs die gelijke belasting aan alle designpunten toewijzen.

In hoofdstuk 6 worden de designs voor logistische mixed effectsmodellen voor binaire longitudinale data beschouwd. Lokale D-Optimale designs worden bestudeerd en worden uitgedrukt als een functie van de regressieparameters. Maximin D-Optimale designs worden beschouwd als een oplossing voor het probleem van parameterafhankelijkheid bij designs voor logistische mixed modellen. Maximin D-Optimale designs met een gegeven interval van parameterwaarden worden door middel van een maximin relatieve efficiëntiemaat berekend. De prestaties van de maximin D-Optimale designs in termen van de maxim efficiency zijn hoog voor een reeks van parameterwaarden die in praktijk gebruikelijk zijn. Verder, worden de maximin designs met verschillende aantallen tijdstippen vergeleken. De resultaten tonen aan dat het optimale aantal herhaalde metingen afhankelijk is van het aantal regressieparameters in het model. De designlocaties verschuiven over het algemeen naar links in vergelijking met de designlocaties voor lineaire mixed effects modellen die gekend zijn in de literatuur. Een gebruikersvriendelijk 
computerprogramma om lokaal en maximin D-Optimale designs voor logistische mixed modellen te verkrijgen, wordt ook beschreven in hoofdstuk.

Acknowledgments: Ik wil graag Marga Doyle en Mickey Chenault bedanken voor hun hulp bij het voorkomen dat mijn eerste samenvatting in het Nederlands 'Lost in Translation' zou zijn. 


\section{Acknowledgments}

Praise the Lord! The thesis is ready and it is time to write the acknowledgments. This thesis would never have been written if it had not been for the assistance, encouragement and support I received from many persons. These acknowledgments try to recognize most of these persons.

In the first place, I would like to thank my promoter prof. dr. Martijn Berger. I am most grateful for your confidence, kindness and patience with me in the course of my entire $\mathrm{PhD}$ project. After I had been selected for the $\mathrm{PhD}$ position, you made tremendous efforts to get the legal papers and get me to the Netherlands. Ever since I came to the Netherlands, you have made sure that everything was fine with my stay in the Netherlands. We have had a number of conversations about research and social life that I value a lot. Your guidance and valuable comments made my works easier. I consider myself very lucky and most honoured to have been one of your students.

I would like to thank my co-promotor, Dr. Frans Tan for his time and patience and for his friendly approach. I am very grateful for your full support, positive creative ideas, valuable guidance and comments. Your explanations from the basics have opened my eyes to optimal design theories and made this thesis easier.

Parts of this thesis have appeared or will appear as articles. The valuable comments by the editors and reviewers have lead to improvements and have been incorporated into the present version and I would like to thank those editors and reviewers.

I would like to thank the members of the evaluation committee Prof. dr. ir. P.A. van den Brandt, Dr. G. van Breukelen, Prof. dr. P. Goos, Prof. dr. G. Verbeke and Prof. dr. H. de Vries for their useful comments and discussions on the manuscript of the thesis.

Everybody at the department of Methodology and statistics, Maastricht University has been helpful to me in my stays in Maastricht and thank you all. Marga Doyle, thank you for your encouragement and managing the administrative issues I have gone through. I have gone sometimes through hasty bureaucratic paths. And thank you for your help in those paths. I had nice room mates Shirley and Baerbel whom I have shared many ideas. Thank you for your encouragements and discussions we had around our similar projects.

I am blessed with support and encouragements from many people in my stays in Maastricht. W/o Merdia Issa, Rael Mulenhe, Kistete (Hawi) Mulenhe, W/o Etetu Ayele (with the whole family), Hanna Debele, Semhar Tekeste, Tewelde Bahta, Mohamed Ali 
Ahmed, Suleyka Hussein Ali, Dr. Herma Roebertsen (the whole family) made our stays in Maastricht easier and comfortable. Thank you all for your support in one or the other way. My especial thanks also go to the members and pastors of the Redeemed Christian Church of God: Covenant Life Parish and Immanuelkerk in Maastricht for their spiritual, moral and social support at different stages of my stay in Maastricht.

I thank my long time friend Dr. Wendimagegn Ghidey and his wife Birkenesh Alemeneh, Dr. Asferd Mengesha (Dr. Nestanet Shiferaw), Dr. Taye Hussien, Amanuel Mokenen, Yetemegn K. Michael (Emuye), Doyo Gragn (Mestawot), Dr. Dawit Tadesse, Senni Asfaw (Ato Israel) and yemisrach (Miser) mekbib for their continuous moral support. I also owe my special thanks to many of my friends who assisted me in my long journey regardless of the geographical distance, including, Yonas Melaku, Eskinder Zwede (Tigist), Neway Mola, Dr. Cherenet Mohamed (Dina), Tsegaye Desalegn (Netsi), Drs. Kinfemichael Alemu and Solomon Chefo, Eba Alemayehu, W/o Messeret Tadesse and Fasil Tessema.

I am thankful to my family and relatives: Ato Bekele Tekle, W/o Tezerash Bayu, W/o Dinbulo Beyene, Alemtsaye Maru, Tekalign Maru, Bekalu Kebede (Etagegn), Menaleshwa Abaye, Bekele Regassa, Genet Cherenet (Ato Bulti), Tadele Beyene (Banche), Dagne Gezahegn (Bogalech), Kebebush Bekele, Abenezer Dagne, Sisay Bogale, W/o Bizunesh Aga, Azeb Wendimu, Mokenen Gelagle, Natinael Mokenen, Seble Gelagle, Dr. Zebene Kifle, Ato Maru Adamu (Tiruwork and the whole family), Bogale Bekabel and Fikadu Endeshaw for their love, encouragement, advice and supports, even though some of them are still wondering what I am doing in Europe.

Finally, I would like to thank my lovely wife Mihiret Anteneh for her love, patience and supports. If it were not for you, I do not think this long journey would be finished. I also apologize to you and our hospitable son Hanniel for the times not spent with you. God bless you all. 


\section{Curriculum Vitae}

Fetene Bekele Tekle was born on May 12, 1974 in Mettu, Ethiopia. He attended Mettu comprehensive secondary high school from 1988 to 1991. Subsequently, he started studying statistics as a major and computer science as a minor at Addis Ababa University (AAU), Ethiopia. He obtained his Bsc degree from the same university in 1995 and started working on the same year as an assistant lecturer at Jimma University (JU), Ethiopia. In addition to teaching responsibilities, he served as a manager of computer center of JU. He joined graduate school of AAU in 1998 and obtained his masters degree in statistics in June 2000. Then, he worked as a lecturer of Biostatistics at public health facility of JU until September 2001. He won the Vlaamse interuniversitaire Raad (Vl.I.R.) scholarship and joined Hasselt University (the former Limburgs Universitair Centrum), Belgium in September 2001. He specialized and obtained masters degrees in Applied Statistics and Biostatistics from Hasselt University in 2002 and 2003, respectively. In 2003, he returned back to Ethiopia and resumed teaching Biostatistics at JU. He married his beautiful girlfriend in 2004 and started his $\mathrm{PhD}$ study at the department of methodology and statistics, Maastricht University at the same year. The birth of his cheerful son, Hanniel, in August 2007 is one of his happiest moments in Maastricht. In June 2008 he started working as an assistant professor at the department of methodology and statistics, Tilburg University. 